\title{
Affective Objectification as the Perception of Intent To Be or Have an Object
}

Richard Baxter ${ }^{*}$, ,

:2915/350 William St, Melbourne VIC 3000, Australia

* richardbrucebaxter@gmail.com

\begin{abstract}
The underling mechanism of sexual objectification requires identification. Cognitive objectification likely stems from gender specific motivations [1. It is theorised that affective objectification is the adaptive subconscious (1) perception of intent to (2) be or (3) have an object (for males and females respectively). Four studies were conducted with 1800 USA participants. The studies presented visual stimuli representing a male, female, and male with female with minimal (typically provocative) clothing. Affective objectification was measured via self-report sensual affect (S1) and arousal (S1-S4). For both affective objectification measures of study S1, a significant effect of manipulating perception of intent to be an object (1) was confirmed for males between all negative manipulation conditions (relabelling the female images: ballet dancer/friend), the neutral condition (no labels), and the positive manipulation condition (relabelling the female images: nightclub dancer). All studies (S1-S4) confirmed that males were significantly more affected by a female stimulus than females (H2C). Studies S1B and S4B (supported by trends from S1A, S3 and S4A) confirmed that for males, the affect generated by an opposite sex stimulus was significantly influenced by the absence of an adjacent same sex stimulus (H2A). Furthermore, the effect was only significant and consistently observed for females with stimuli containing complete upper body exposure (no shirt; S2) and high body shape exposure (tight pants; S4), not high lower body exposure (short robe; S1-S4). All studies confirmed that males were significantly more affected by opposite sex visual stimuli than females (H2B). Similarly however, this was only consistently observed for high lower body exposure stimuli (S1-S6), not complete upper body exposure (S2) or high body shape exposure (S4). These findings support a male specific perception of intent to be a sexually accessible object (2). Moreover, study S4 found that high intent but low body shape exposure stimuli (short robe) did not produce significantly more affect for males than low intent but high body shape exposure stimuli (tight pants). The results suggest that perception of intent may be a gender specific moderator rather than the sole cause of affective objectification (innate affective biases in object recognition should be considered).
\end{abstract}

\section{Author Summary}

\section{Introduction}

Sexual objectification is typically defined as the perception or treatment of an individual as an object rather than a person [2, 3. While similar definitions have been maintained in the empirical literature, there has been no consensus developed around 
the psychological mechanism of objectification. Objectification is typically portrayed in terms of behavioural, cognitive, or affective phenomena (See S1 Appendix) [4].

Target gender differences have been identified in objectification. Most studies find that female targets are more prone to being objectified than males $1,4,8$. This is supported by research suggesting that there may be object recognition biases in the interpretation of the female body [9-11].

Likewise, participant gender differences have been identified in objectification and subsequently linked to differences in motive. Vaes et al. [1 has found that sexual attraction drives objectification of females by males whereas females appear to be influenced by the desire to distance themselves away from abnormal or threatening presentations of their gender. Puvia and Vaes [8] have likewise argued that competition may drive objectification in females. Furthermore, gender differences in the interpretation of sexualised stimuli (unwanted sexual advances) have been identified [12, 13. Research also suggests that mortality salience interacts with reproductive salience to produce self-objectification in females [14, which might exacerbate the mechanistic objectification of females [5].

Yet if objectification has an evolutionary (adaptive) basis, then it is likely to have unconscious and affective roots. These in particular should display gender differences. Objectification has been associated with modifications in affect (arousal) in males [1, but gender differences in its affective mechanism have not been systematically investigated.

Furthermore, it has been found that objectification operates on perception of intent. The perceived agency of a target is reduced not only through perceived nakedness, but perceived suggestiveness (sexual pose) [15]. This direct intent manipulation has however only been conducted with female participants and female stimuli, and differences in sexual affect have not been measured. Similarly, Morris and Goldenberg [5] suggest that manipulating the sexual behaviour of stimuli might produce measurable differences in objectification (at least in terms of animalistic dehumanisation). Moreover, an assumption of intent dependence is implicit in the behavioural framing of objectification, for example clothing type [1, 15] 17], facial dominance [15, 17], eye gaze [18], and presentation with sexually degrading language [19].

Finally, evolutionary psychology can provide some estimates on the nature of affective objectification across gender, and these appear to have intent dependencies. Such assumes that the encoded stimuli associations have evolved based on copulation with a genetically superior mate. In the case of males, their physical goal has evolved to be a fertile female reproductive system, and the perception of direct evidence of their compliance/desire to provide this (a presented healthy body). In the case of females, their physical goal has evolved to be a sufficiently competitive and determined mate, and the perception of direct evidence of a successful one (unchallenged touch).

Moreover, male reproductive psychology is expected to be particularly relevant to sexual objectification. The evolutionary argument is based on the environment of evolutionary adaptedness for a prototypical sexually dimorphic species (male competition for access to females). Traits can only be encoded if there is selective pressure on them. Male psychological traits encouraging reproduction are strongly selected for as males who do not possess these will not reproduce in their natural environment. There is an excess of male gametes in sexually reproductive species, and not all males need reproduce to satisfy the maximum reproductive capacity of the population. Female psychological traits encouraging reproduction (e.g. offspring caring behaviours) are less strongly selected for as females will be selected for reproduction by their male counterparts regardless of their possession of these traits. Reproduction requires highly valuable female wombs (and gametes); months of their reproductive opportunity. Thus, their reproductive psychology bears less on whether they will 
reproduce.

Affective objectification is theorised to be (1) the subconscious perception of intent to (2) be or (3) have an object; for males and females respectively. The perception of intent to have an object corresponds to (is the subconscious basis for) the desire to be physically desired in females; and as such is more contextually dependent than the perception of intent to be an object of desire. The observation of intent to have an object generally contains information about the object of the intent (for example, male interaction with self or another female), whereas the observation of intent to be an object does not generally (in contemporary western society) contain information about the object of the intent (although it is likely to be affected by such knowledge if available; for example, female interaction with self or another male).

This definition treats socialised cognitions/attitudes as artefacts of a more primitive affective mechanism. For example, the subject [4] is relevant to the extent it represents or does not impede this intention. Moreover, it does not reduce the effect to sexual attractiveness or conscious desire. Such objectification is however perhaps an abstraction (misassociation) of natural subconscious desire during intercourse. For example, it may equate to the desire for completion/conception under the presumption of intercourse (from which may arise similar intentions). Affective objectification might therefore correspond to the adaptive and subconscious stimuli responses active during intercourse (but where they have been triggered in an arbitrary context).

Furthermore, it is based on a highly adaptive perception of intent to procreate (to be or have a sexual object). It is theorised that we are sensitive to the subconscious (non-exclusively unconscious) perception of intent. Conscious recognition may trigger unconscious associations, but the same associations may be triggered without conscious awareness. Likewise, the unconscious associations are not affectively neutral and are therefore non-exclusively unconscious. It is possible the (male) brain does not easily distinguish (or care to distinguish) between the origin of the intent, as it has evolved to seek (/respond to) reproductive opportunity. Its basis being a carnal drive for power/dominance (restoration of the primacy of long evolved instincts to the negation of more complex goals directed by more modern physical or social systems). For example, intent might be inferred from a perceived lack of intent to dignify/love/respect the subject, including the presentation of negligence, mindlessness (automata based dehumanisation), or vice (subjective insignificance).

Such a model explains why appearance does not uniquely drive objectification for males, but is a contextually dependent phenomenon. Examples include experience of exotic tribes, males and females in love, family, close friendships, revelations of manipulation, dehumanisation and denial of intent, the spellbound nature of the effect and its sensitivity to evidence to the contrary including humour, historic and cultural variations in the indication of intent, framing, positioning, capturing, experiencing another's affect (desire), the sexual compatibility of the environment including instantaneously sexually viable versus other clothing types, activity/purpose/goals including medical settings, explicit intent and verbal confirmation including the intentional faking of desire exhibited during sexual relations, evidence of unwillingness to be an object including obesity, dependence on arousal/awareness, sexual priming, the dominance and health of the observer, portrayal of innocence and promiscuity, bodily modification, enhancement, exposure, emphasis, distortion, or highlighting, autonomy promotion versus subjugation, etc. Furthermore the perception of intent could be a socialised or conditioned association.

It shall be recognised here that affective objectification may be a dual process. Perception of intent might only be a modifier of affective objectification, as opposed to its (exclusive) source. The source may lie in even more basic processing, such as precoded, adaptive object recognition. The research here should facilitate some first 
estimates to be drawn on their relative contributions.

\section{Studies}

In order to examine the theory, four studies were conducted. These studies measured the sensual affect or arousal generated by experiencing a set of visual stimuli.

It was first generally hypothesised (1) that objectification is the perception of intent to be/have an object. It was next generally hypothesised $(2 / 3)$ that males/females are differentially affected by stimuli suggesting a female giving herself as an object versus a male seeking an object. The general hypothesis 3 has not been tested by this work, as this requires contextually dependent and ethically challenging manipulations (to convey the intent to have an object as directed towards female participants individually).

Data were obtained using the crowd source service CrowdFlower. All four studies were conducted with USA participants. A total of 1800 participants contributed to the studies (1518 final; after performance filtering). Some participants contributed to multiple studies, but their data were not excluded from the analysis. Out of the total 1800 (1518 final) participants surveyed, 1007 (818 final) were identified as being unique (as determined by their Crowdflower Worker IDs).

Ethics approval by an ethics committee was not required for the studies (they are classified as negligible risk research according to the NHMRC). CrowdFlower contributors (and those of associated channels) voluntarily completed an electronic survey titled "Affect Survey". They were provided task instructions before completing the survey, and were informed by the crowd source program that they were only paid if they completed the survey. They were also informed by the crowd source program that they could exit at any time (e.g. "If you'd like to try another task or would rather go enjoy the sunshine, you can leave a task at any time by clicking here"). The data were obtained and analysed anonymously.

\section{Study 1}

The first study measured self-report sensual affect (S1A) or arousal (S1B) from exposure to visual stimuli containing either a male, female, or male with female (close together). These measures were chosen as non-explicit references to affective objectification (sexual arousal).

Participants were presented with three (3) images and asked to rate the 'sensual affect' or 'arousal' experienced by each image. Images were silhouettes based on black (foreground) and white (background) abstractions of the Pioneer Plaque, with the addition of minimal (typically provocative) clothing. Dress was normalised based on body (reproductive system) shape; see S2 Appendix. It did not accentuate either the male or the female reproductive system (to prevent a perception of intent bias in this respect). Both males and females were adorned with a short robe, as this has commonly (and successfully) been used in objectification manipulations. Stimuli were completely filled based on evidence the human vision system relies on contrast recognition (meaning adaptations thereof should be tied to shape recognition). The images were not designed to induce high levels of sensual affect/arousal (as they did not require this).

Furthermore, five (5) manipulation conditions were chosen. The same three images were presented in each manipulation condition, but they were labelled according to their manipulation (acting as an implicit identifier of the persons imaged and therefore as an intent manipulation). 'Regular' clothing control conditions were not used as this research was only looking for gender/intent manipulation differences and the theory is not dependent on the type of stimuli (only the relative intention perceived).

A set of experimental hypotheses were developed based on the general hypotheses. 
It was first hypothesised (H1) that males would be differentially more affected by the female stimuli in the high perception of intent manipulation condition (M4) than in the neutral (M3) and low perception of intent manipulation conditions (M1/M2).

It was next hypothesised (H2A) that for males, the affect rating for the female stimulus would be greater than the affect rating for the male with female stimulus. This was based on an inferred higher probability of the female being single and wishing to be an attractive object for them. Likewise, it was hypothesised (H2B), that males would be more affected by the female stimulus than females would be affected by the male stimulus. This was based on males being sensitive to the perception of intent to be an object and therefore sexually suggestive visual stimuli. Finally, it was hypothesised $(\mathrm{H} 2 \mathrm{C})$ that males would be more affected by the female stimulus than females. This functioned as a basic evolutionary affect manipulation check.

Study S1B was designed to a) replicate the effect using a different measure of affective objectification, and b) dissociate interpersonal affect from the self-report sensual affect measure used in S1A. Study H1B also decreased the hip fat content of the female images relative to study S1A, to normalise the fat content with respect to the male counterpart. This change was justified considering interstudy differences in absolute affect were not being tested.

\section{Method}

Participants 500/500 participants undertook study S1A and S1B respectively. 84/61 participants were removed from the study S1A/S1B data analysis based on a performance filter (see S3 Appendix). This resulted in a final 416/439 participants. Participants were paid 20/30 US cents for their participation in a survey. This was evaluated by the participants as a significant amount of pay (rated on average 4.6/4.6 out of 5.0, with overall survey satisfaction 4.7/4.7 out of 5.0). 340 (306 final) participants were identified as having contributed to previous studies in S1B.

Materials The surveys from studies S1A and S1B both consisted of 5 questions. See S2 Appendix for a list of all stimuli, survey questions and response options. The first three questions asked 'How sensually affected/aroused are you by the above image?' with respect to either an image of a male, an image of a female, or an image of a male with a female (close together). There were 5 manipulation conditions (including a control condition). In the non-control manipulation conditions, the images were labelled according to their manipulation. Study S1 employed ten level Likert scales to measure self-report sensual affect, in the form of a 10 point (radio button) horizontal scale. This ranged from "not affected/aroused at all" (1) to "extremely affected/aroused" (10). Although each option (radio button) was numbered, they were only named at the extremes of the horizontal scale. Study S1 also measured gender (male or female).

Both S1A and S1B were split into five parts, each for a given manipulation. For each condition (M1 through to M5), the images were labelled 'Ballet Dancer' (M1), 'Family Friend' (M2), ' (M3/control), 'Nightclub Dancer' (M4), and 'Exotic Dancer' (M5) respectively. The first two conditions (M1, M2) were considered to be intent negative textual manipulations, and the last two (M4, M5) were considered to be intent positive textual manipulations, while condition M3 was taken to be an intent neutral control condition. Condition M1 consisted of 142/101 (108 final; 54/39 male, 54/45 female) participants. Condition M2 consisted of 87/100 (78/93 final; 27/53 male, 51/40 female) participants. Condition M3 consisted of 79/98 (64/88 final; 23/35 male, 41/53 female) participants. Condition M4 consisted of 117/98 (99/84 final; 46/34 male, 53/50 female) participants. Condition M5 consisted of 75/103 (67/90 final; 32/33 male, 35/57 female) participants. The indices of conditions M2 and M4 in S1A are switched relative to the 
order in which the conditions were conducted (as their indices have been aligned with those of study S1B for comparison purposes).

M1 and M2 were considered to be intent negative manipulations, M4 was considered to be an intent positive manipulation, and M3 was taken to be an intent neutral control condition. M5 was ignored in all analyses as it was speculated to provide an overall intent negative manipulation; i.e. the combined visual and textual stimuli convey negative intent. The presence of any clothing (albeit minimal) on an exotic dancer is atypical and conveys modesty rather than intent to reproduce. Compare the stimuli presented in S2 Appendix (short robe) to that of a prototypical exotic dancer (naked/underwear). The results from both S1A and S1B confirmed this discrepancy.

Procedure For both S1A and S1B, a survey was delivered to participants as an online form (see S2 Appendix). Instructions were provided within the survey itself.

\section{Results}

For male participants, the means and standard errors for the self-report sensual affect ratings (S1A) are listed in Fig. 1. For female participants, the means and standard errors for the self-report sensual affect ratings (S1A) are listed in Fig. 2 For male participants, the means and standard errors for the self-report arousal ratings (S1B) are listed in Fig. 3. For female participants, the means and standard errors for the self-report arousal ratings (S1B) are listed in Fig. 4

Male participants $(n=182)$

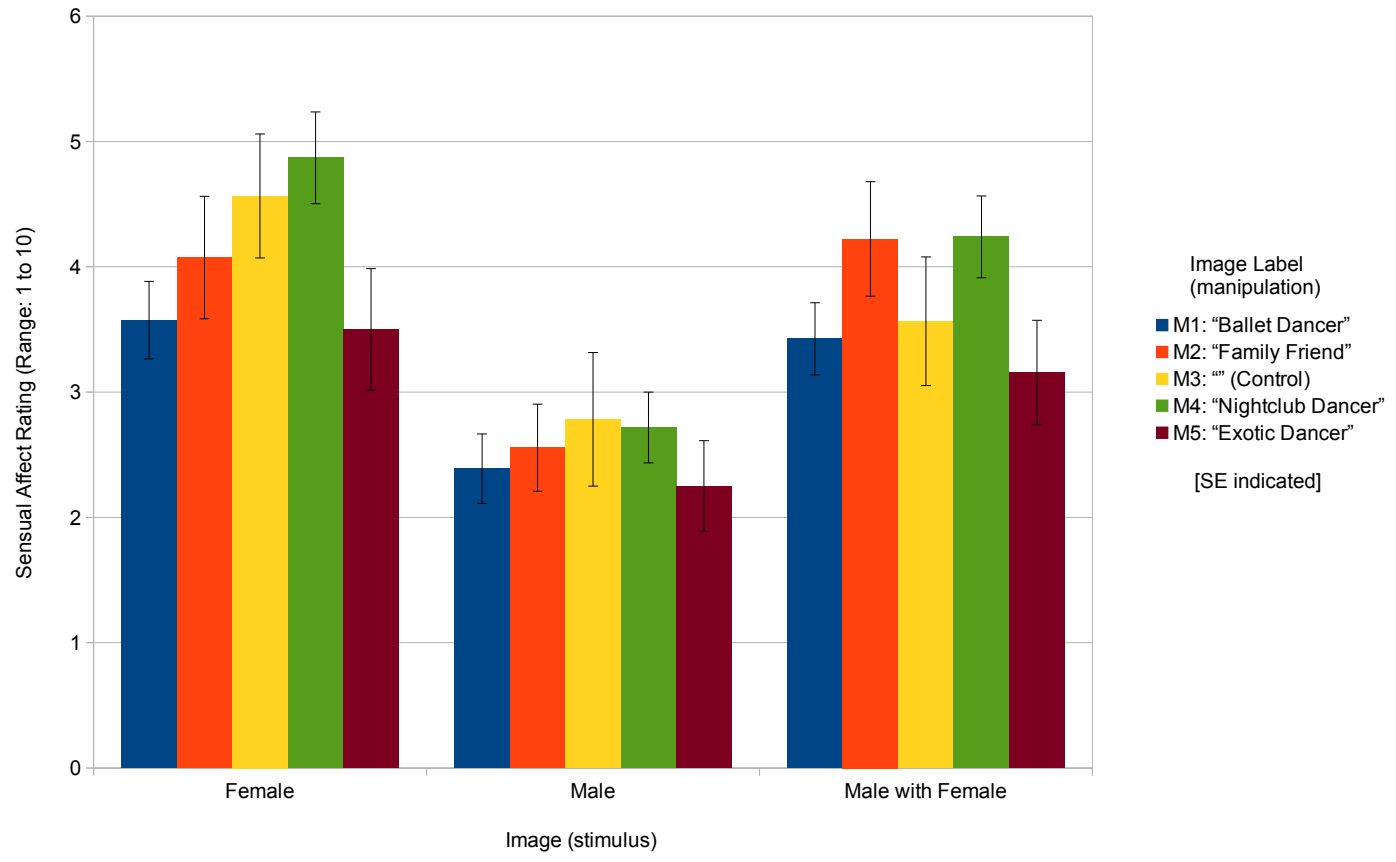

Figure 1. Study 1A mean self-report sensual affect ratings for male participants (for each image stimulus: male/female/male with female, and for each manipulation condition). Standard errors marked. 1 corresponds to 'not affected at all', 10 corresponds to 'extremely affected'. 


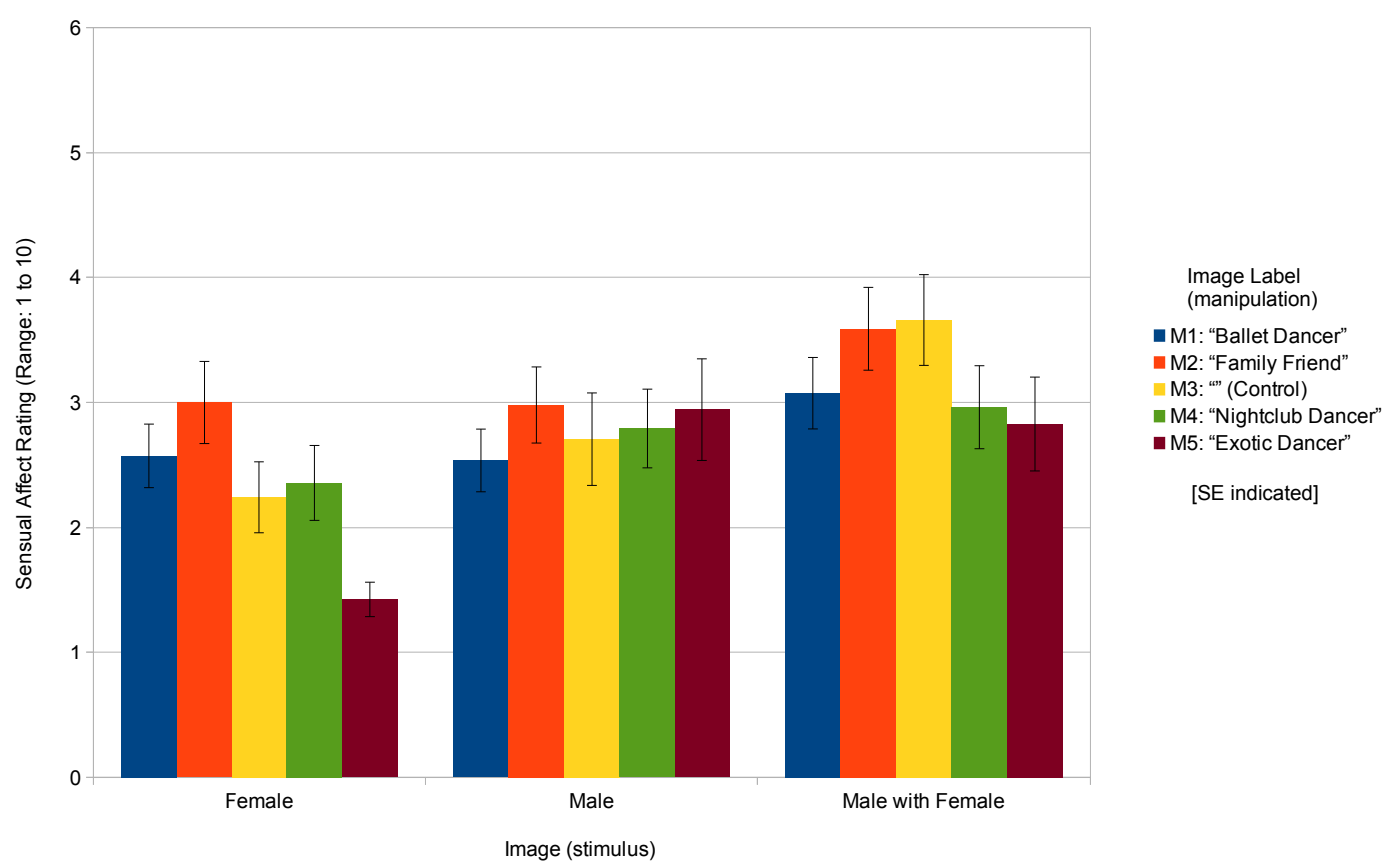

Figure 2. Study 1A mean self-report sensual affect ratings for female participants (for each image stimulus: male/female/male with female, and for each manipulation condition). Standard errors marked. 1 corresponds to 'not affected at all', 10 corresponds to 'extremely affected'.

Hypothesis $\mathrm{H} 1$ was supported by both S1A and S1B. See Table 1. One-way ANOVAs with planned contrasts (M1:-0.5, M2:-0.5, M3:0, M4:1) were used to test the hypothesis. In S1A, males were differentially more affected by their M4 intent positive manipulation than their M3 neutral and M1/M2 intent negative manipulations, $t(146)$ $=2.31, p=.022$. In S1B, males were differentially more affected by their M4 intent positive manipulation than their M3 neutral and M1/M2 intent negative manipulations, $t(157)=2.03, p=.044$. In both S1A and S1B, the M5 manipulation (exotic dancer) did not demonstrate a positive effect.

Hypothesis H2A was partially supported by S1A and fully supported by S1B. See Table 2. For S1A, and in partial accordance with H2A, for males, the affect rating for the female stimulus was significantly greater than the affect rating for the male with female stimulus, across manipulation conditions M4 and M5, and marginally significantly greater across M3. The only condition to not exhibit the expected trend was M2. For S1B, and in accordance with $\mathrm{H} 2 \mathrm{~A}$, for males, the affect rating for the female stimulus was significantly greater than the affect rating for the male with female stimulus, across all manipulation conditions.

Conversely, in S1A it was noted that for females, the affect rating for the male stimulus was not significantly greater than the affect rating for the male with female stimulus, for all manipulation conditions. Moreover, it trended in the opposite direction for four of these conditions, in which three of these were significant. Likewise, in S1B it was noted that for females, the affect rating for the male stimulus was not significantly greater than the affect rating for the male with female stimulus, although it trended in 


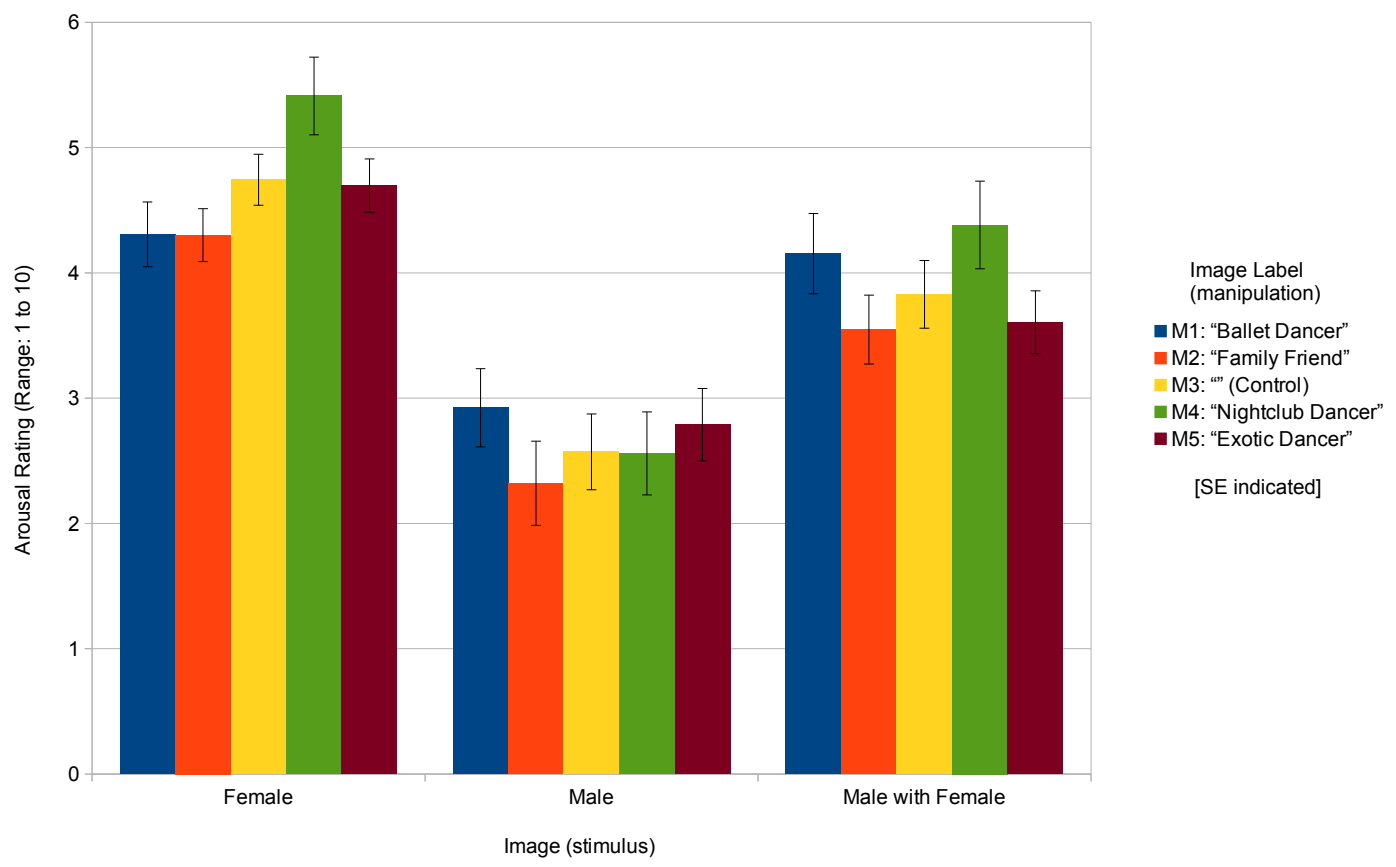

Figure 3. Study 1B mean self-report arousal ratings for male participants (for each image stimulus: male/female/male with female, and for each manipulation condition). Standard errors marked. 1 corresponds to 'not aroused at all', 10 corresponds to 'extremely aroused'.

this direction in four out of five manipulation conditions. See Table 5.

Hypothesis H2B was partially supported by S1A and fully supported by S1B. See Table 3 For S1A, and in partial accordance with H2B, males were significantly more affected by the female stimulus than females were affected by the male stimulus, across all manipulation conditions besides M5. For S1B, and in accordance with $\mathrm{H} 2 \mathrm{~B}$, males were significantly more affected by the female stimulus than females were affected by the male stimulus, across all manipulation conditions.

Hypothesis H2C was partially supported by S1A and fully supported by S1B. See Table 4 For S1A, and in partial accordance with H2C, males were significantly more affected by the female stimulus than females, across all manipulation conditions besides M2. For S1B, and in accordance with $\mathrm{H} 2 \mathrm{C}$, males were significantly more affected by the female stimulus than females, across all manipulation conditions.

The results for hypothesis $\mathrm{H} 1$ are listed in Table 1. The results for hypotheses H2A, $\mathrm{H} 2 \mathrm{~B}$, and $\mathrm{H} 2 \mathrm{C}$ are listed in Tables 2,3 , and 4 .

Table 1. Results for Hypothesis H1: male self-report affect based on female stimuli containing positive versus negative intent manipulations

\begin{tabular}{|l|r|r|r|r|r|}
\hline Study & Manipulation (contrasts) & $t$ & $d f$ & $p$ & trend? \\
\hline S1A & M1 $+\mathrm{M} 2, \mathrm{M} 3$, and M4 & 2.31 & 146 & .022 & yes \\
\hline S1B & M1 $+\mathrm{M} 2, \mathrm{M} 3$, and M4 & 2.03 & 157 & .044 & yes \\
\hline
\end{tabular}




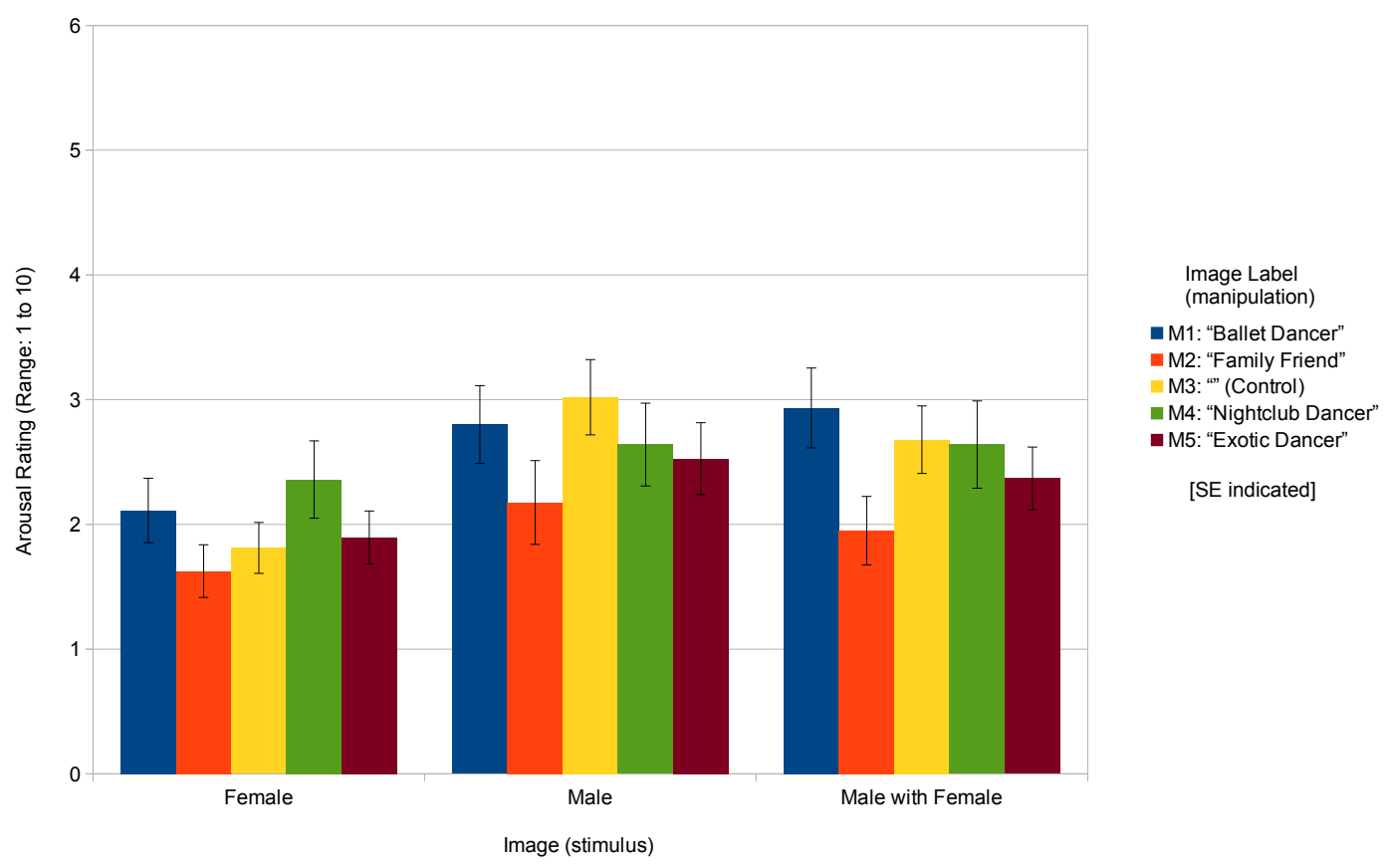

Figure 4. Study 1B mean self-report arousal ratings for female participants (for each image stimulus: male/female/male with female, and for each manipulation condition). Standard errors marked. 1 corresponds to 'not aroused at all', 10 corresponds to 'extremely aroused'.

\section{Discussion}

Study S1 obtained evidence in favour of (1) the influence of perception of intent on affective objectification. Hypothesis H1 was supported by both S1A and S1B, measuring sensual affect and arousal across their intent manipulations.

For study S1A, it was believed that manipulation 2 (family friend) produced high interpersonal but low sexual affect, and the male with female stimuli produced high interpersonal effect in general (influencing the effect sizes of hypothesis H2A). Sensual affect was chosen in study S1A based on its non-explicit reference to sexual arousal. Study S1B was therefore designed to separate sexual affect from non-sexual interpersonal affect (while retaining non sexually explicit language); by measuring generic arousal.

For both $\mathrm{S} 1 \mathrm{~A}$ and $\mathrm{S} 1 \mathrm{~B}$, it is expected that the relative perception of intent was lower for the M5 (exotic dancer) condition based on a prior dress expectations (noting that all manipulation conditions used the same visual stimuli). The results are concordant with the M5 condition being seen as more modest than is usually expected from such a character. Such aligns with the theory (1).

Study S1 likewise produced evidence for the theory's claims for (2) male versus female differences in affect (affective objectification) when experiencing the "objectification" (behavioural objectification/apparent self-objectification) of another; specifically, the perception of intent to be an object. The results suggest that such differences indeed exist. Hypotheses $\mathrm{H} 2 \mathrm{~A}, \mathrm{H} 2 \mathrm{~B}$, and $\mathrm{H} 2 \mathrm{C}$ were partially supported by 
Table 2. Results for Hypothesis H2A: male self-report affect based on female versus male with female stimuli

\begin{tabular}{|l|r|r|r|r|r|}
\hline Study & Manipulation & $t$ & $d f$ & $p$ & trend? \\
\hline S1A & M1 & 0.49 & 53 & .630 & yes \\
& M2 & -0.39 & 26 & .697 & no \\
& M3 & 1.81 & 22 & .084 & yes \\
& M4 & 2.03 & 45 & .048 & yes \\
& M5 & 0.72 & 31 & .478 & yes \\
\hline S1B & M1 & 0.42 & 38 & .676 & yes \\
& M2 & 2.75 & 52 & .008 & yes \\
& M3 & 2.74 & 34 & .010 & yes \\
& M4 & 2.25 & 33 & .031 & yes \\
& M5 & 2.49 & 32 & .018 & yes \\
\hline S2 & M1 & 0.18 & 30 & .861 & yes \\
& M2 & 0.56 & 32 & .582 & yes \\
\hline S3 & M1 & 1.39 & 37 & .173 & yes \\
& M2 & 1.07 & 32 & .292 & yes \\
\hline S4A & M1 & 1.15 & 35 & .260 & yes \\
& M2 & 0.71 & 23 & .485 & yes \\
\hline S4B & M1 & 2.06 & 47 & .045 & yes \\
& M2 & 2.82 & 59 & .007 & yes \\
\hline
\end{tabular}

Table 3. Results for Hypothesis H2B: male versus female self-report affect based on opposite sex stimuli

\begin{tabular}{|l|r|r|r|r|r|}
\hline Study & Manipulation & $t$ & $d f$ & $p$ & trend? \\
\hline S1A & M1 & 2.61 & 101.6 & .011 & yes \\
& M2 & 2.00 & 76 & .050 & yes \\
& M3 & 3.02 & 62 & .004 & yes \\
& M4 & 4.33 & 97 & $<.001$ & yes \\
& M5 & 0.89 & 65 & .379 & yes \\
\hline S1B & M1 & 2.87 & 72.3 & .005 & yes \\
& M2 & 4.44 & 91 & $<.001$ & yes \\
& M3 & 3.18 & 86 & .002 & yes \\
& M4 & 4.71 & 82 & $<.001$ & yes \\
& M5 & 4.08 & 88 & $<.001$ & yes \\
\hline S2 & M1 & 0.67 & 72 & .505 & yes \\
& M2 & 3.52 & 59 & .001 & yes \\
\hline S3 & M1 & 2.16 & 71 & .034 & yes \\
& M2 & 3.28 & 53 & .002 & yes \\
\hline S4A & M1 & -0.70 & 72.0 & .485 & no \\
& M2 & 1.98 & 79.0 & .051 & yes \\
\hline S4B & M1 & 1.75 & 99.0 & .083 & yes \\
& M2 & 2.27 & 92.0 & .025 & yes \\
\hline
\end{tabular}

study S1A and fully supported by study S1B.

Firstly, males were more affected by an isolated female stimulus than a male with female stimulus (H2A). According to the theory, this is caused by an indication of single/engaged status; and the modification of the perceived probability of intent to be an object (for them personally) accordingly. It is possible that the effect observed in $\mathrm{H} 2 \mathrm{~A}$ has contributed to the western tradition of chaperones.

Contrarily, females were either more affected by a male and female pair than an isolated male stimulus (S1A), or less affected by the isolated male stimulus over the 
Table 4. Results for Hypothesis H2C: male versus female self-report affect based on female stimuli

\begin{tabular}{|l|r|r|r|r|r|}
\hline Study & Manipulation & $t$ & $d f$ & $p$ & trend? \\
\hline S1A & M1 & 2.50 & 106 & .014 & yes \\
& M2 & 1.87 & 76 & .065 & yes \\
& M3 & 4.39 & 62 & $<.001$ & yes \\
& M4 & 5.36 & 97 & $<.001$ & yes \\
& M5 & 4.26 & 65 & $<.001$ & yes \\
\hline S1B & M1 & 4.44 & 64.1 & $<.001$ & yes \\
& M2 & 6.83 & 84.3 & $<.001$ & yes \\
& M3 & 5.55 & 46.0 & $<.001$ & yes \\
& M4 & 5.02 & 55.7 & $<.001$ & yes \\
& M5 & 5.24 & 44.1 & $<.001$ & yes \\
\hline S2 & M1 & 4.90 & 72.0 & $<.001$ & yes \\
& M2 & 4.09 & 56.3 & $<.001$ & yes \\
\hline S3 & M1 & 3.56 & 62.2 & $<.001$ & yes \\
& M2 & 4.50 & 46.8 & $<.001$ & yes \\
\hline S4A & M1 & 3.00 & 62.6 & .004 & yes \\
& M2 & 3.31 & 31.1 & .002 & yes \\
\hline S4B & M1 & 4.82 & 87.6 & $<.001$ & yes \\
& M2 & 2.52 & 92.0 & .014 & yes \\
\hline
\end{tabular}

Table 5. Female self-report affect based on male versus male with female stimuli

\begin{tabular}{|l|r|r|r|r|r|r|r|}
\hline study & manipulation & $t$ & $d f$ & $p$ & trend? & measure & significant? \\
\hline S1A & M1 & -2.33 & 53 & .023 & no & sensual affect & yes \\
& M2 & -2.37 & 50 & .022 & no & sensual affect & yes \\
& M3 & -3.64 & 40 & $<.001$ & no & sensual affect & yes \\
& M4 & -0.89 & 52 & .380 & no & sensual affect & no \\
& M5 & 0.56 & 34 & .579 & yes & sensual affect & no \\
\hline S1B & M1 & -0.95 & 44 & .349 & no & arousal & no \\
& M2 & 1.05 & 39 & .298 & yes & arousal & no \\
& M3 & 1.67 & 52 & .101 & yes & arousal & no \\
& M4 & 0.00 & 49 & 1.000 & yes & arousal & no \\
& M5 & 0.89 & 56 & .375 & yes & arousal & no \\
\hline S2 & M1 & 4.46 & 42 & $<.001$ & yes & arousal & yes \\
& M2 & -1.87 & 45 & .068 & no & arousal & no \\
\hline S3 & M1 & 0.43 & 38 & .670 & yes & arousal & no \\
& M2 & 0.09 & 47 & .926 & yes & arousal & no \\
\hline S4A & M1 & 3.46 & 37 & .001 & yes & arousal & yes \\
& M2 & 1.27 & 56 & .209 & yes & arousal & no \\
\hline S4B & M1 & 3.54 & 52 & .001 & yes & arousal & yes \\
& M2 & -0.49 & 33 & .625 & no & arousal & no \\
\hline
\end{tabular}

male and female pair as compared to males with their respective opposite sex stimuli (S1B). In study S1A, this reverse (rather than the expected null or weaker) effect observed for females was likely due to a confound in the sensual affect measure (interpersonal affect). As such, it vindicated the decision for study S1B (and all subsequent studies) to measure arousal directly.

Likewise, males were more affected by a sexually suggestive female visual stimulus than females were affected by a sexually suggestive male visual stimulus (H2B). This provides direct evidence for general hypothesis 2 . Although measurements have not been made in the context of affective objectification, it aligns with findings that females 
are more prone to objectification than males [4, 5]. The result is not however obviously compatible with findings to the contrary; that objectification (the denial of mind) can occur equally for female and male stimuli [15, 17. These discrepancies are possibly attributable to the cognitive (as opposed to affective) responses being measured (which could have been different). Yet it can be noted that these studies both used shirtless men in their objectification conditions.

There might therefore be a gender bias in affect with respect to the experience of opposite sex upper versus lower body exposure. This follows from general hypothesis 2 . Females would be expected to be differentially less affected than males by stimuli containing an exposed (or at least, instantaneously sexually viable) opposite sex reproductive system. Study 2 was created to both confirm this tentative experimental hypothesis (H3A) and rule out the possibility that such an effect (if existent) adversely affects the generalisability of the study S1 hypothesis $\mathrm{H} 2 \mathrm{~B}$ result. It did this by checking whether females were no more affected by opposite sex upper body exposure than males (H3B).

Furthermore, study S1 confirmed that males were more affected by a female stimulus than females (H2C). This served as a between stimuli manipulation check and supports the validity of the affective objectification construct (in that the self-report sensual affect aligns with what basic evolutionary theory would predict). Furthermore, it concords with previous research highlighting a gender bias in self-report arousal to female stimuli [1]. Likewise, it aligns with experiments demonstrating that male gaze (relative to that of female participants) is drawn to low waist-to-hip ratio female targets [20]. It also confirms speculation that males are more likely than females to sexualise an appearance salient female [5].

All hypothesis $\mathrm{H} 2$ results support the relevance of gender in affective objectification.

Although evidence has been presented in favour of general hypothesis 2, study S1 cannot test general hypothesis 3 , the perception of intent to have an object. This properly requires non-visual stimuli (e.g. touch, dialogue); in which experimental designs become increasingly unethical and difficult to fake. (Note a common theme between male and female affective objectification under this model is that they both involve lies with respect to humanity).

\section{Study 2}

Study S2 measured self-report arousal data based on stimuli with complete upper body exposure (versus the high lower body exposure of studies S1A and S1B). In accordance with general hypothesis 2 , it was hypothesised (H3A) that males would be more sensitive to lower than upper opposite sex body exposure (based on female reproductive system exposure/sexual object presentation), but this effect would not be observed for females. Furthermore, it was speculated that females might even be more sensitive to upper than lower opposite sex body exposure (based on a potential indication of desire to have an object through the display of strength; see general hypothesis 3), although this was not hypothesised.

Study S2 was primarily designed to rule out the possibility that the gender disparity observed for hypothesis H2B (in both studies S1A and S1B) was an artefact of a particular area of the body being exposed (and did not hold on average). It was therefore additionally hypothesised (H3B) that females would be no more attracted to upper body exposure of males than males are of females.

The study S2 data were also used to further test hypothesis H2.

Study S2 used a female hip fat content level halfway between that of study S1A and study $\mathrm{S} 1 \mathrm{~B}$. 


\section{Method}

Participants 197 participants undertook study S2. 44 participants were removed from the study S2 data analysis based on a performance filter (see S3 Appendix). This resulted in a final 153 participants. Participants were paid 30 US cents for their participation in a survey. This was evaluated by the participants as a significant amount of pay (rated on average 4.6 out of 5.0, with overall survey satisfaction 4.4 out of 5.0). 104 (79 final) participants were identified as having contributed to previous studies.

Materials The questions of the S2 survey were based on those used in study S1B. There were 2 manipulation conditions, and the images were not labelled (to match the control conditions from studies S1A and S1B). See S2 Appendix for a list of all stimuli, survey questions and response options.

Study S2 was split into two parts, each for a given manipulation (M1/M2). M1 tested participant responses to complete upper body exposure. It consisted of 100 (74 final; 31 male, 43 female) participants. M2 tested participant responses to high lower body exposure. It consisted of 97 (79 final; 33 male, 46 female) participants.

Procedure A survey was delivered to participants as an online form (see S2 Appendix. Instructions were provided within the survey itself.

\section{Results}

For male participants, the means and standard errors for the self-report arousal ratings are listed in Fig. 5. For female participants, the means and standard errors for the self-report arousal ratings are listed in Fig. 6 .

In accordance with hypothesis $\mathrm{H3A}$, males were marginally significantly more affected by stimuli containing an exposed sexual object (lower body/female reproductive system) than otherwise (upper body exposure), $t(62)=-1.93, p=.058$. Likewise, in accordance with H3A this effect was not observed for females. Females were non-significantly more affected by opposite sex upper body exposure than opposite sex lower body exposure, $t(72)=0.67, p=.505$.

Likewise, in accordance with hypothesis H3B, females were no more aroused by opposite sex upper body exposure than males, $t(87)=0.92, p=.361$. Furthermore, in this sample, males remained more attracted to the female stimulus than vice versa (suggesting no reason to question the validity of $\mathrm{H} 2 \mathrm{~B} ; M=3.16$ versus $M=2.81$ ).

Hypothesis 2 was partially supported by study S2. Contrary to H2A, for males, the affect rating for the female stimulus was not significantly greater than the affect rating for the male with female stimulus, in either manipulation condition (although it trended in the right direction for the sexually suggestive control condition). Yet it was noted that for females, the affect rating for the male stimulus was significantly greater than the affect rating for the male with female stimulus, for the complete upper body exposure manipulation condition (and trended in the same direction in the control condition) - see Table 5.

Likewise, contrary to H2B, males were not significantly more affected by the female stimulus than females were affected by the male stimulus, for the complete upper body exposure manipulation condition. It was however significant for the sexually suggestive control condition.

Finally, in accordance with $\mathrm{H} 2 \mathrm{C}$, males were significantly more affected by the female stimulus than females in both manipulation conditions.

The results for hypotheses $\mathrm{H} 2 \mathrm{~A}, \mathrm{H} 2 \mathrm{~B}$, and $\mathrm{H} 2 \mathrm{C}$ are listed in Tables 2,3 , and 4 . 


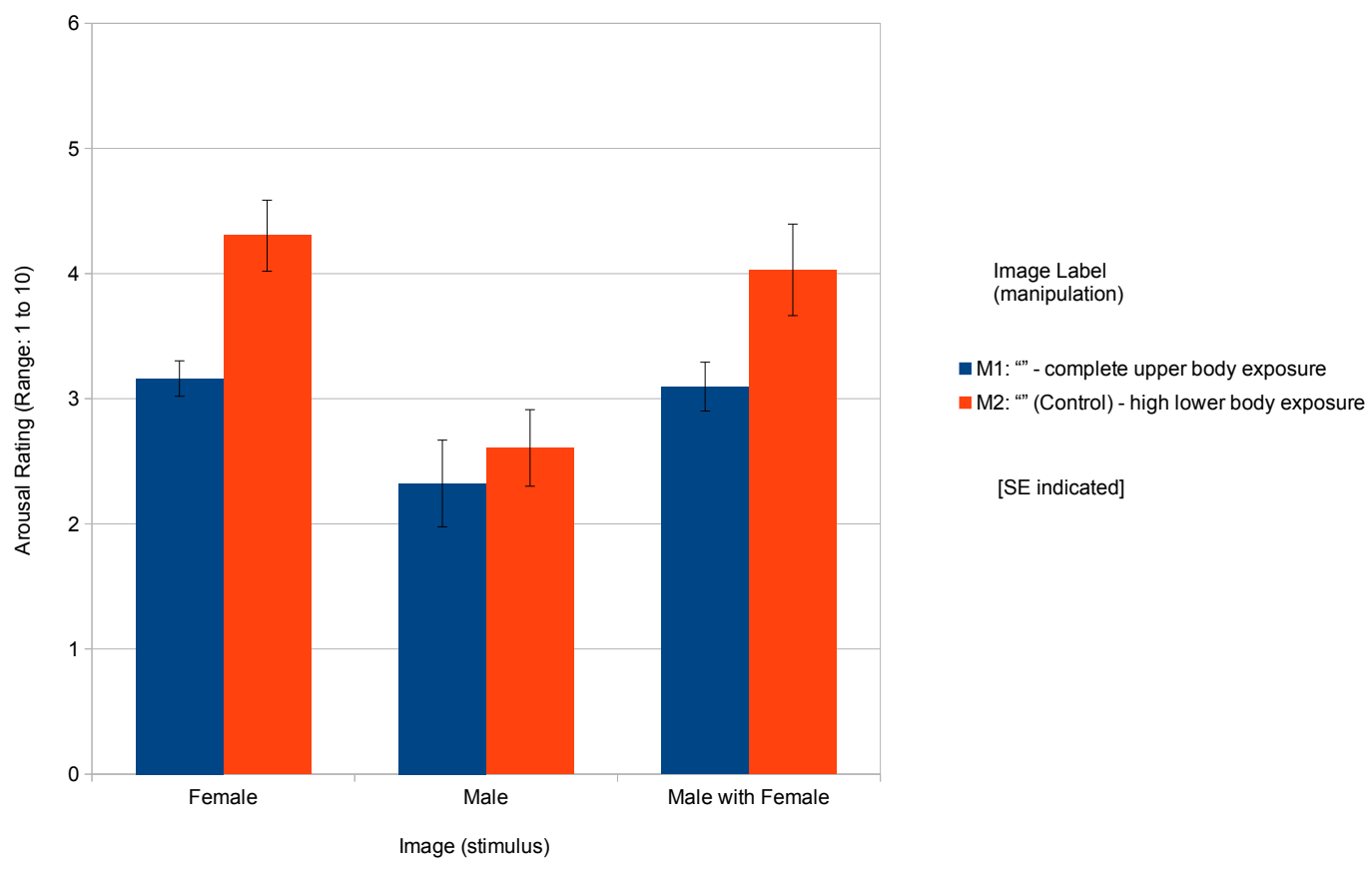

Figure 5. Study S2 mean self-report arousal ratings for male participants (for each image stimulus: male/female/male with female, and for each manipulation condition: upper vs lower body exposure). Standard errors marked. 1 corresponds to 'not aroused at all', 10 corresponds to 'extremely aroused'.

\section{Discussion}

Study S2 found evidence of a suspected interaction between gender and type of body exposure. Males were more affected by lower body exposure than upper body exposure, while this was not observed for females (hypothesis H3A). This supports general hypothesis 2 , in that males are specifically sensitive to the perception of intent to be an object, while females are not sensitive to such information. Likewise, the observation concords with the evolutionary basis of their low waist-to-hip ratio attraction bias [21].

Furthermore, study S2 confirmed that females were no more affected by opposite sex upper body exposure than males (hypothesis H3B). As such, the results from studies S1A and S1B for hypothesis H2B stand. Males appear to remain differentially more attracted to opposite sex sexually suggestive visual stimuli.

The upper body affect measurements from study S2 (versus the affect measurements of S1) concord with attentional bias measurements based on full body exposure [22], in which male and female attention is drawn similarly to opposite sex stimuli. Likewise, they concord with findings that suggest affective responses to the presentation of sexually explicit content do not differ greatly between gender [23]. Yet they cannot be used to dispute the male bias in visual stimuli induced affective objectification (H2B) observed in studies S1A and S1B. There remains little evidence that females can be made to respond to opposite sex visual stimuli equally (without substantially clothing/limiting exposure of the female reproductive system).

It can also be noted that this research provides limited support for speculation that 


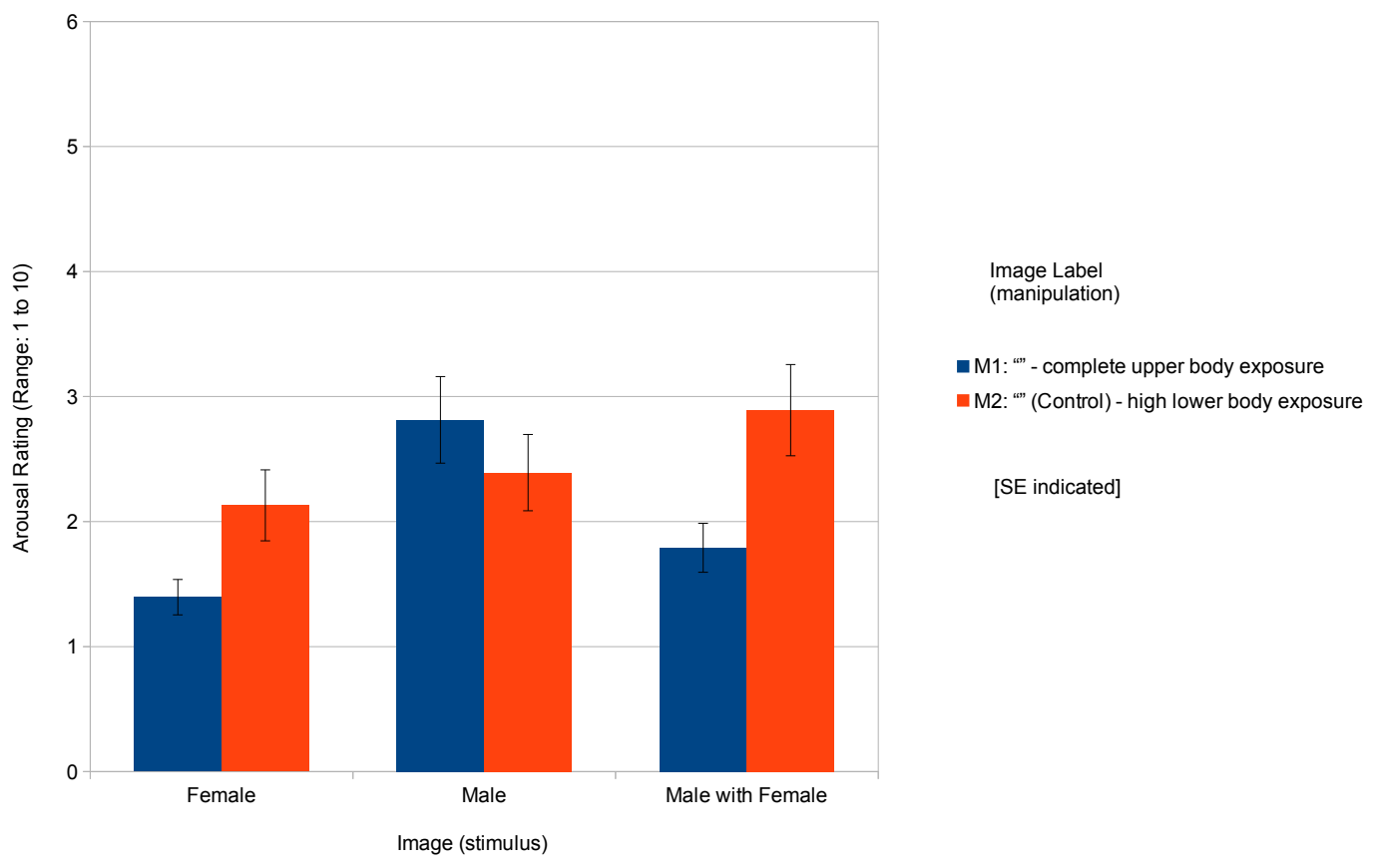

Figure 6. Study S2 mean self-report arousal ratings for female participants (for each image stimulus: male/female/male with female, and for each manipulation condition: upper vs lower body exposure). Standard errors marked. 1 corresponds to 'not aroused at all', 10 corresponds to 'extremely aroused'.

women might be more affected by upper body exposure than lower body exposure. Such might have been predicted from general hypothesis 3 (based on an interpretation of intent to behaviourally objectify/have an object, from the display of another's capacity to behaviourally objectify/strength). Likewise, it might have been predicted from their shoulder-to-waist ratio attraction bias [21]. The results however align with the hypothesised requirement of a target specific indication of intent to have an object (see general hypothesis 3), and a divergence of female sexual affect with respect to visual activation (see general hypothesis 2 ).

Finally, only the control condition of study $\mathrm{S} 2$ could be used to confirm the validity of hypothesis H2. The trends observed for H2A and H2B were consistent with the hypothesis and all previous studies, yet they were both entirely non-significant for the upper body exposure condition. $\mathrm{H} 2 \mathrm{C}$ was however verified in both conditions. The results suggest that the effect observed for hypotheses $\mathrm{H} 2 \mathrm{~A}$ and $\mathrm{H} 2 \mathrm{~B}$ is dependent on the reproductive relevance of the stimuli. Such concords with the perception of intent theory, in that perception of intent can only be conveyed visually with intent relevant stimuli (where in the case of males, this is the intent to be a sexually accessible object; see general hypothesis 2 ). 


\section{Study 3}

Study S3 measured self-report arousal data based on stimuli with no head hair (versus the stimuli with head hair used in studies S1A and S1B). Study S3 otherwise used the same stimuli as study S1B.

Study S3 was designed to further test general hypotheses 1 and 2, that affective objectification for males is affected by the perception of intent to be an object. It was expected that the presence of long head hair on a female stimulus would greater support the (potential) perception of intent to be an object than the absence of such head hair. In accordance with general hypotheses 1 and 2, it was hypothesised (H4) that the female stimulus containing long hair would produce greater arousal in males than the female stimulus containing no hair.

Like study S2, the data were also used to further test hypothesis H2.

\section{Method}

Participants 201 new participants undertook study S3. 41 participants were removed from the study S3 data analysis based on a performance filter (see S3 Appendix). This resulted in a final 160 participants. Participants were paid 30 US cents for their participation in a survey. This was evaluated by the participants as a significant amount of pay (rated on average 4.6 out of 5.0, with overall survey satisfaction 4.5 out of 5.0). 60 (55 final) participants were identified as having contributed to previous studies.

Materials The questions of the S3 survey were again based on those used in study S1B. Like study S2, there were 2 manipulation conditions, and the images were not labelled (to match the control conditions from studies S1A and S1B). See S2 Appendix for a list of all stimuli, survey questions and response options.

Study S3 was split into two parts, each for a given manipulation (M1/M2). M1 tested participant responses to images without head hair. It consisted of 100 (77 final; 38 male, 39 female) participants. M2 tested participant responses to images with head hair. It consisted of 101 ( 81 final; 33 male, 48 female) participants.

Procedure A survey was delivered to participants as an online form (see $\mathrm{S} 2$ Appendix). Instructions were provided within the survey itself.

\section{Results}

For male participants, the means and standard errors for the self-report arousal ratings are listed in Fig. 7. For female participants, the means and standard errors for the self-report arousal ratings are listed in Fig. 8

Contrary to hypothesis $\mathrm{H} 4$, there was no significant difference between the male self-report arousal ratings of the female images with long and no hair, $t(69)=-1.08, p=$ .282. Males were however on average more aroused by the female images with long head hair than those with no head hair. Likewise, when compared to the control stimulus in study $\mathrm{S} 1 \mathrm{~B}$, the effect was significant, $t(71)=2.01, p=.048$.

Hypothesis $\mathrm{H} 2$ was partially supported. With respect to $\mathrm{H} 2 \mathrm{~A}$, for males, the affect rating for the female stimulus was not significantly greater than the affect rating for the male with female stimulus, although this trended in the right direction in both manipulation conditions. Contrarily, it was noted that for females, the affect rating for the male stimulus was not significantly greater than the affect rating for the male with female stimulus, and there was no substantive trend in either manipulation condition see Table 5 . 


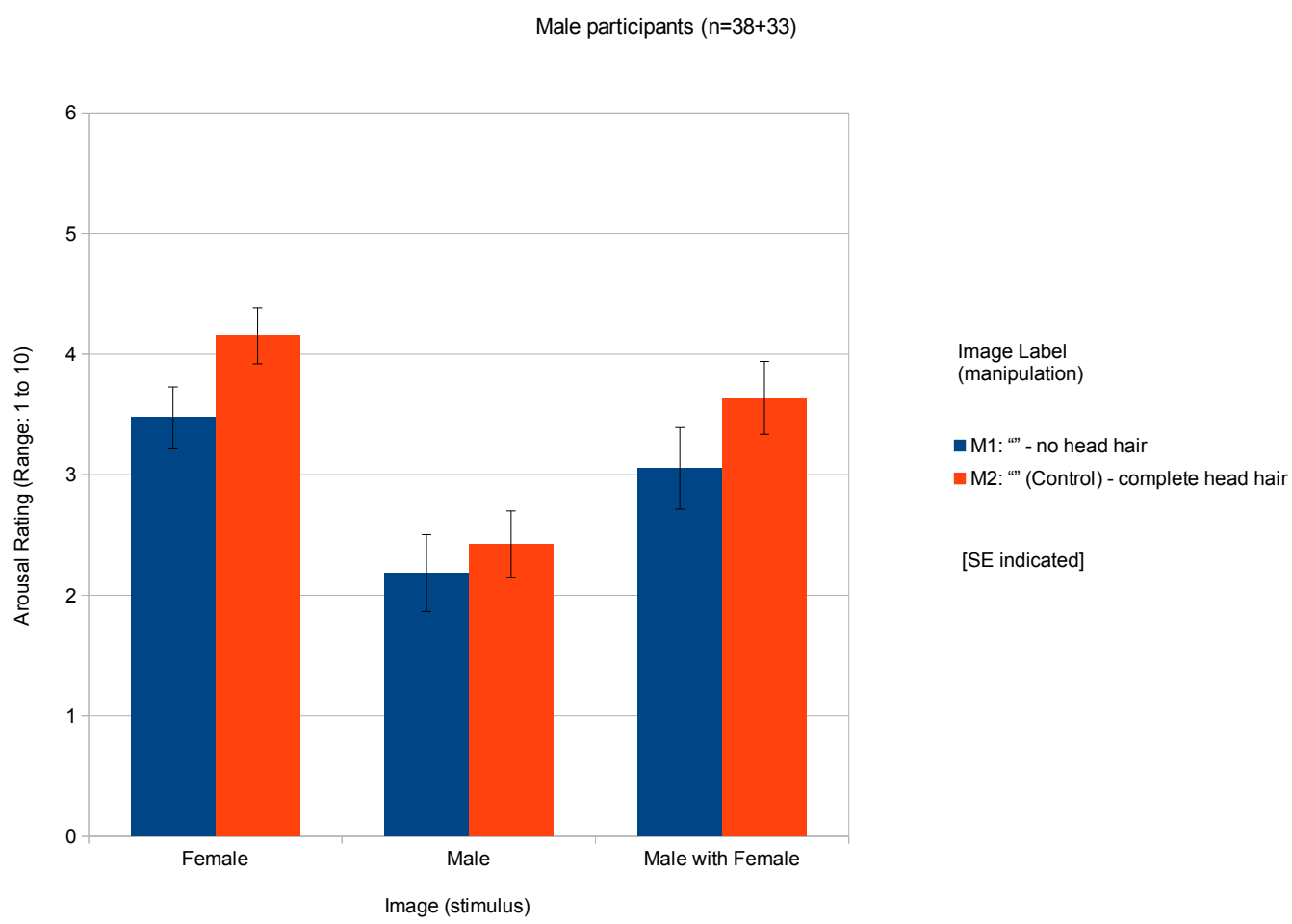

Figure 7. Study S3 mean self-report arousal ratings for male participants (for each image stimulus: male/female/male with female, and for each manipulation condition: no head hair vs head hair). Standard errors marked. 1 corresponds to 'not aroused at all', 10 corresponds to 'extremely aroused'.

In accordance with $\mathrm{H} 2 \mathrm{~B}$, males were significantly more affected by the female stimulus than females were affected by the male stimulus, in both manipulation conditions.

Finally, and in accordance with $\mathrm{H} 2 \mathrm{C}$, males were significantly more affected by the female stimulus than females, in both manipulation conditions.

The results for hypotheses $\mathrm{H} 2 \mathrm{~A}, \mathrm{H} 2 \mathrm{~B}$, and $\mathrm{H} 2 \mathrm{C}$ are listed in Tables 2,3 , and 4 .

\section{Discussion}

Study S3 manipulated perception of intent to be an object by modifying the presence of head hair on a female stimulus. It found limited evidence to support the validity of hypothesis $\mathrm{H} 4$ (and in this respect general hypotheses 1 and 2). Male self-report arousal ratings of the sexually suggestive female images with long hair were however greater than those with no hair, and this was significant when compared to the study S1B control data.

It is possible that males have evolved to find long hair to be an attractive property in a female mate [24], and have affect laden object recognition systems devoted to the task. Swami et al. 25] however found no significant main effect of hair length on female attractiveness, while systematically varying hair length, hair colour and skin tone. It is doubtful therefore that hair length attraction could be coded at a low (object

recognition) level if physical attractiveness does not consistently depend on hair length. This suggests that hair length might function as a confirmation or contradiction of prior 


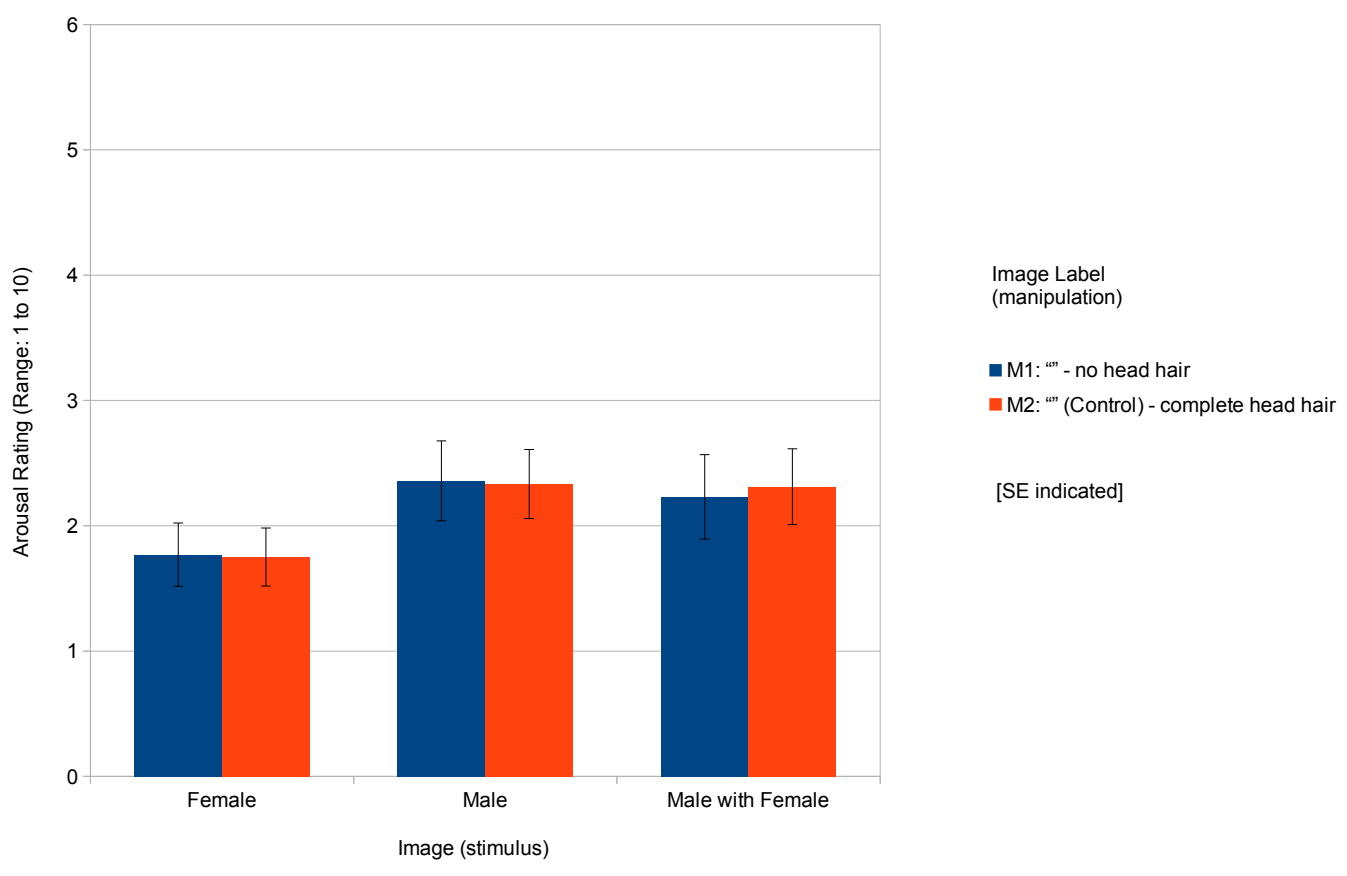

Figure 8. Study S3 mean self-report arousal ratings for female participants (for each image stimulus: male/female/male with female, and for each manipulation condition: no head hair vs head hair). Standard errors marked. 1 corresponds to 'not aroused at all', 10 corresponds to 'extremely aroused'.

perception of intent to be an object rather than general attractiveness.

Furthermore, it is possible the images created without head hair were in some ways defective with respect to normative head shape, thereby reducing the overall arousal experienced by participants (for reasons other than perception of intent to be an object).

Likewise, it is possible that the trend observed was a statistical anomaly, given the non-significance of the primary analysis. When compared to the control stimuli of study S1B however, the effect was significant.

Finally, study S3 confirmed the validity of hypothesis H2. The trend observed for H2A was consistent with the hypothesis and all previous studies, albeit non-significant. All other comparisons were significant.

Based on the relatively weak effects observed for hypothesis $\mathrm{H} 1$ and $\mathrm{H} 4$ in studies S1A, S1B, and S3, it was thought that the two theories of affective objectification (intent versus innate object recognition bias) might be disentangled by presenting participants with images containing either high body shape exposure but low provocativeness (tight but full body clothing) or high provocativeness but low body shape exposure (classical short robe stimuli). Study S4 was created to test if the intent manipulation outweighed the object recognition manipulation. 


\section{Study 4}

Study S4 measured self-report arousal data based on images containing either high body shape exposure but low provocativeness (tight but full body clothing) or high provocativeness but low body shape exposure (classical short robe stimuli). The first class of images were designed to especially invoke object recognition, while the second class of images were designed to especially invoke intent perception.

Study S4 was split into two parts, S4A and S4B, where S4B was designed to replicate the results from $\mathrm{S} 4 \mathrm{~A}$.

Study S4A used the same stimuli as study S1A, except that in a first condition the robes were replaced with tight pants, and in a second curvature was added to the female robe (to endow it with a more natural look under gravity). Study S4B used the same stimuli as study S4A, except that unlike study S4A, no modifications were made to the curvature of the female robe stimuli (relative to S1A).

Study S4 was designed to further test general hypotheses 1 and 2, that affective objectification is affected by the perception of intent to be an object. It was hypothesised (H5) that the female images containing a sexually exposed reproductive system but with relatively low body shape exposure (short robe) would produce greater arousal in males than images containing high body shape exposure but relatively low sexual exposure (tight pants).

Like studies S2 and S3, the data were also used to further test hypothesis 2.

\section{Method}

Participants 200/202 participants undertook study S4A and S4B respectively. 45/7 participants were removed from the study S4A/S4B data analysis based on a performance filter (see S3 Appendix). This resulted in a final 155/195 participants. Participants were paid $30 / 30$ US cents for their participation in a survey. This was evaluated by the participants as a significant amount of pay (rated on average $4.5 / 4.3$ out of 5.0, with overall survey satisfaction 4.6/4.7 out of 5.0). 123/166 (100/160 final) participants were identified as having contributed to previous studies.

Materials The questions of the S4A and S4B surveys were again based on those used in study S1B. There were 2 manipulation conditions, and the images were not labelled in either (to match the control conditions from studies S1A and S1B). See S2 Appendix for a list of all stimuli, survey questions and response options.

Both study S4A and S4B were split into two parts, each for a given manipulation (M1/M2). M1 tested participant responses to images with tight pants. It consisted of 100/102 (74/101 final; 36/48 male, 38/53 female) participants. M2 tested participant responses to images with a short robe (like studies 1 and 2). It consisted of 100/100 (81/94 final; $24 / 60$ male, 57/34 female) participants. In study S4B, the indices of the conditions are switched relative to the order in which the conditions were conducted (as their indices have been aligned with those of study S4A for comparison purposes).

Procedure For both S4A and S4B, a survey was delivered to participants as an online form (see S2 Appendix). Instructions were provided within the survey itself.

\section{Results}

For S4A male participants, the means and standard errors for the self-report arousal ratings are listed in Fig. 9. For S4A female participants, the means and standard errors for the self-report arousal ratings are listed in Fig. 10. For S4B male participants, the means and standard errors for the self-report arousal ratings are listed in Fig. 11. For 
S4B female participants, the means and standard errors for the self-report arousal ratings are listed in Fig. 12

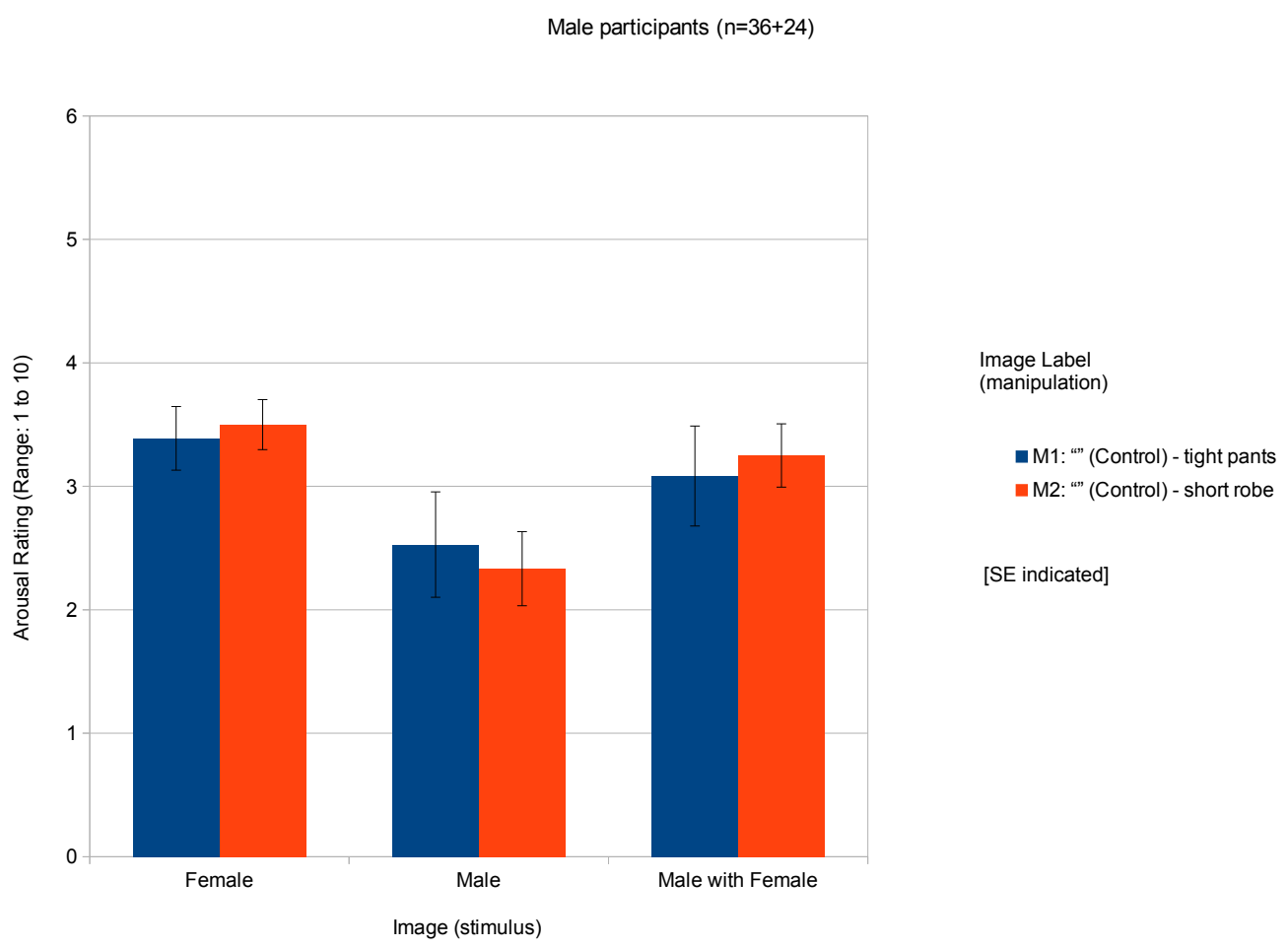

Figure 9. Study S4A mean self-report arousal ratings for male participants (for each image stimulus: male/female/male with female, and for each manipulation condition: tight clothing vs short robe). Standard errors marked. 1 corresponds to 'not aroused at all', 10 corresponds to 'extremely aroused'.

For S4A, and contrary to hypothesis $\mathrm{H} 5$, there was no significant difference between the male self-report arousal ratings of the female images with tight pants and a short robe, $t(58)=-0.18, p=.856$. Likewise for $\mathrm{S} 4 \mathrm{~B}$, and contrary to hypothesis $\mathrm{H} 5$, there was no significant difference between the male self-report arousal ratings of the female images with tight pants and a short robe, $t(106)=-0.36, p=.723$.

Hypothesis H2 was however partially supported by both S4A and S4B.

Hypothesis H2A was partially supported by both S4A and S4B. For S4A, and contrary to H2A, for males, the affect rating for the female stimuli was not significantly greater than the affect rating for the male with female stimuli, across either manipulation condition. Yet the difference trended in the expected direction (for both manipulation conditions). For $\mathrm{S} 4 \mathrm{~B}$, and in accordance with $\mathrm{H} 2 \mathrm{~A}$, for males, the affect rating for the female stimuli was significantly greater than the affect rating for the male with female stimuli, across both manipulation conditions.

Furthermore, in S4A it was noted that for females, the affect rating for the male stimulus was significantly greater than the affect rating for the male with female stimulus, for the tight pants manipulation condition (and trended in the same direction for the short robe condition). Likewise in $\mathrm{S} 4 \mathrm{~B}$, it was noted that for females, the affect rating for the male stimulus was significantly greater than the affect rating for the male with female stimulus, for the tight pants manipulation condition (and trended in the same direction for the short robe condition). See Table 5. 


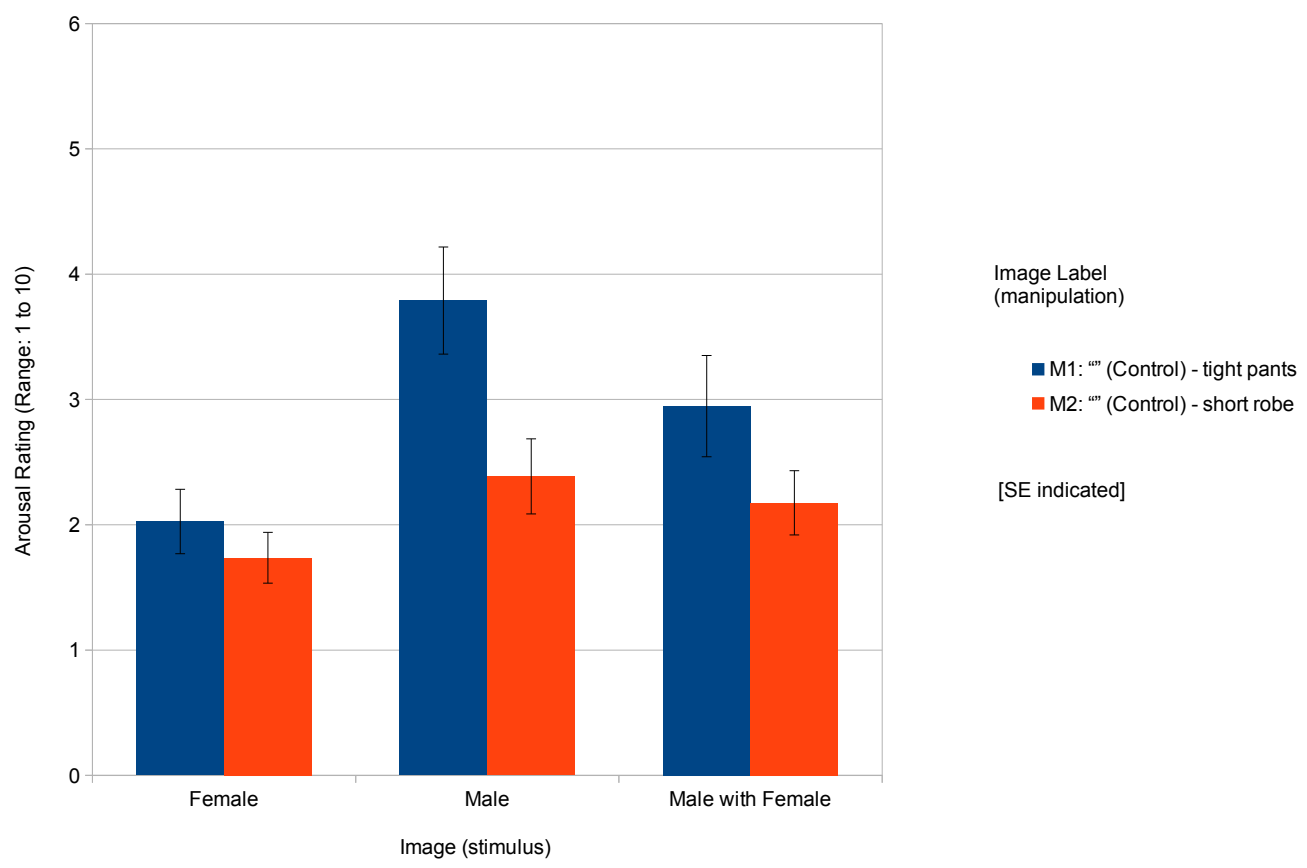

Figure 10. Study S4A mean self-report arousal ratings for female participants (for each image stimulus: male/female/male with female, and for each manipulation condition: tight clothing vs short robe). Standard errors marked. 1 corresponds to 'not aroused at all', 10 corresponds to 'extremely aroused'.

Hypothesis H2B was partially supported by both S4A and S4B. For S4A, and in support of $\mathrm{H} 2 \mathrm{~B}$, in the short robe manipulation condition (M2), males were marginally significantly more affected by the female stimuli than females were affected by the male stimuli; $p=.051$. Yet contrary to H2B, in the tight pants manipulation condition (M1), males were not significantly more affected by the female stimuli than females were affected by the male stimuli. Likewise for $\mathrm{S} 4 \mathrm{~B}$, and in support of $\mathrm{H} 2 \mathrm{~B}$, males were significantly more affected by the female stimuli than females were affected by the male stimuli in the short robe condition (M2). Yet again this was non-significant in the tight pants condition (M1); $p=.083$.

Finally, hypothesis H2C was fully supported by both S4A and S4B. For S4A, and in accordance with $\mathrm{H} 2 \mathrm{C}$, males were significantly more affected by the female stimulus than females. Likewise for S4B, and in accordance with $\mathrm{H} 2 \mathrm{C}$, males were significantly more affected by the female stimulus than females.

The results for hypotheses $\mathrm{H} 2 \mathrm{~A}, \mathrm{H} 2 \mathrm{~B}$, and $\mathrm{H} 2 \mathrm{C}$ are listed in Tables 2,3 , and 4

\section{Discussion}

Study S4 manipulated perception of intent to be an object by modifying the clothing of the female stimulus. Both study S4A and S4B failed to support hypothesis $\mathrm{H} 5$, and in this respect no evidence was provided for general hypotheses 1 and 2 .

Contrary to hypothesis 1 , study S4 suggests that intent independent object 


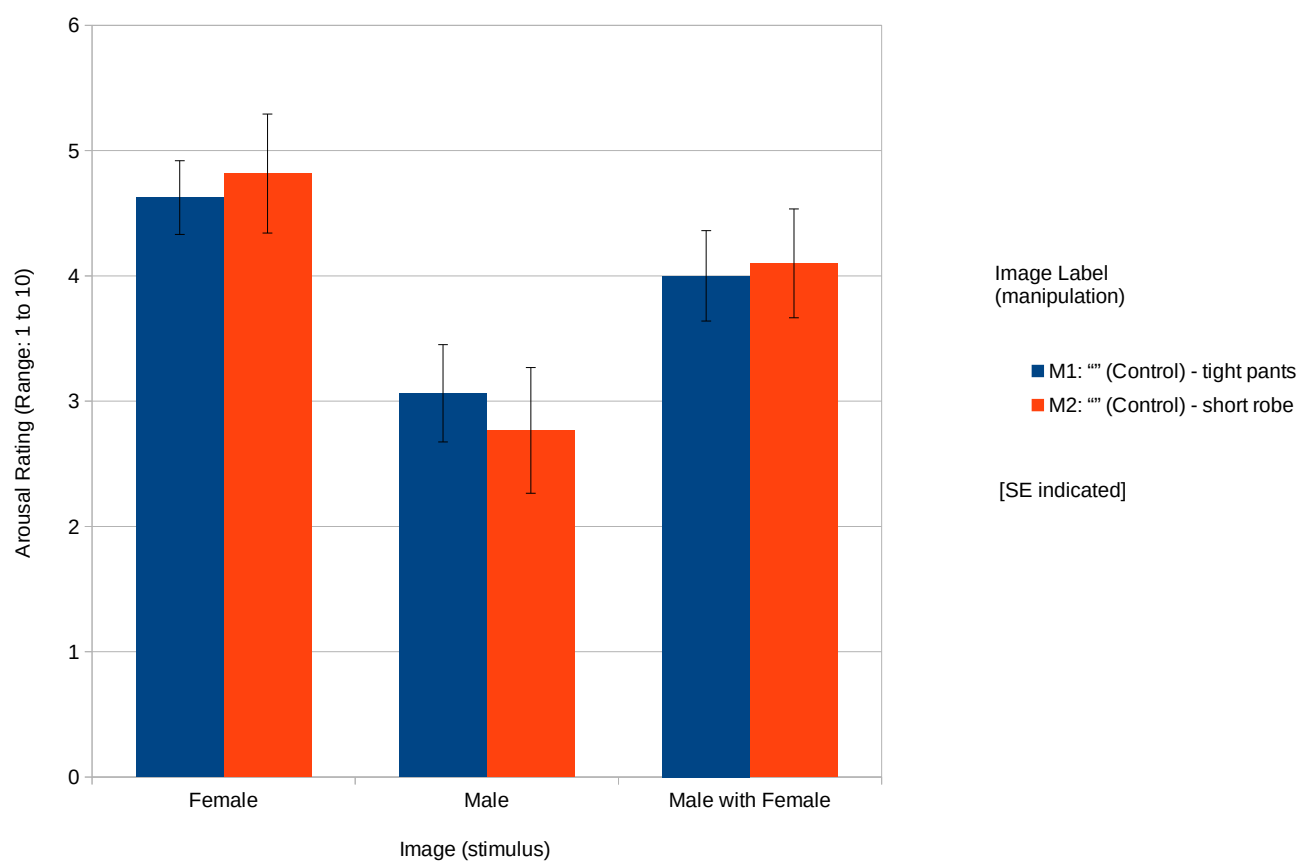

Figure 11. Study S4B mean self-report arousal ratings for male participants (for each image stimulus: male/female/male with female, and for each manipulation condition: tight clothing vs short robe). Standard errors marked. 1 corresponds to 'not aroused at all', 10 corresponds to 'extremely aroused'.

recognition processes are contributing to affective objectification. The difference in affect generated by the high intent (short robe) and high object recognition (tight pants) conditions was not significant (hypothesis H5).

The fact the high object recognition low intent condition did not produce (significantly) more affect than the low object recognition high intent condition could suggest that perception of intent is moderating affective object recognition rather than exclusively facilitating it (in partial agreement with general hypotheses 1 and 2). However, it is not certain that the low object recognition condition (short robe) activated object recognition to a lesser extent than the high object recognition condition (tight pants). The human object recognition system may have been automatically completing the partially occluded innate bodily stimuli in the robe conditions. As such, without a positive result in the intent manipulation, this study cannot be used to support the theory.

Likewise, it is possible that the low perception of intent condition (tight clothing) triggered a conditioned association with intent (provocation of its own). Without eliminating these confounds it is impossible to derive any solid implications for the theory from the study.

Furthermore, it is possible that the provocation typically associated with short robe stimuli is dependent on intent indicators tied to texture rather than reproductive opportunity/exposure (for example surface modifications). Future research should attempt to identify the intent dependencies of various bodily stimuli (independent of object recognition processing). 


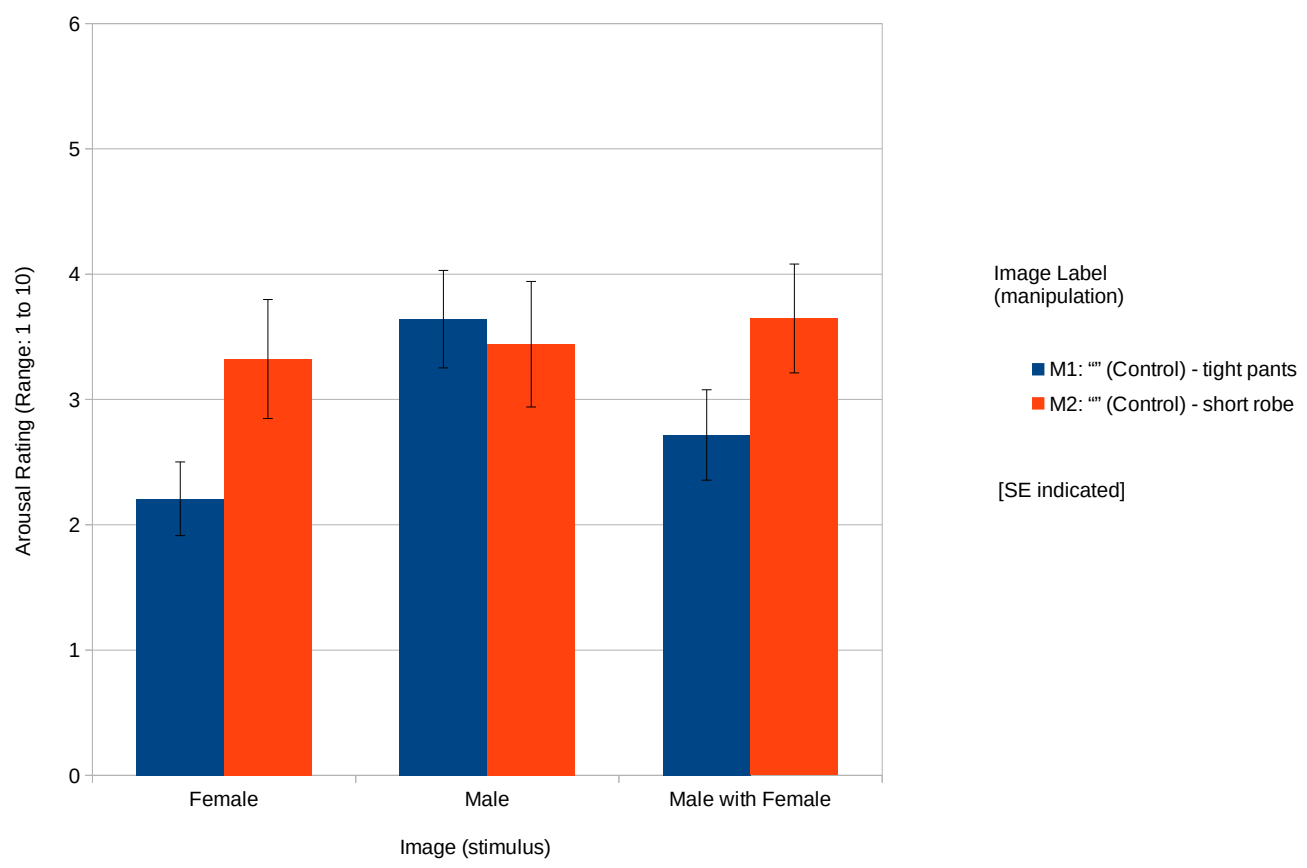

Figure 12. Study S4B mean self-report arousal ratings for female participants (for each image stimulus: male/female/male with female, and for each manipulation condition: tight clothing vs short robe). Standard errors marked. 1 corresponds to 'not aroused at all', 10 corresponds to 'extremely aroused'.

It is also possible that participants mistakenly interpreted the tight pants stimuli as being half naked (considering they were black and white). Additional thickness was not added to the pants (to prevent this) based on a desire not to distort the natural shape of the body relative to the short robe stimuli conditions.

Study S4 however partially confirmed the validity of hypothesis $\mathrm{H} 2$.

H2A was partially supported by S4. For S4A, the trend observed for H2A was consistent with the hypothesis and all previous studies, albeit non-significant for both conditions. For S4B, hypothesis H2A was fully supported by both manipulation conditions.

$\mathrm{H} 2 \mathrm{~B}$ was supported in the low object recognition high intent (short robe) condition, but unsupported in the high object recognition low intent (tight pants) condition. For S4A, males were not significantly more aroused by opposite sex stimuli in the high object recognition low intent condition than females. Only in the sexually suggestive control condition (low object recognition high intent) were males significantly more aroused by opposite sex stimuli than females. Likewise, for S4B, males were not significantly more aroused by female stimuli than females were aroused by male stimuli in the high object recognition low intent condition (although this appeared to be approaching significance). Again, only in the sexually suggestive control condition (low object recognition high intent) were males significantly more aroused by opposite sex stimuli than females.

Taking into account the complete discrepancy observed for hypothesis H2B in the 
upper body exposure/high object recognition conditions of studies S2 and S4A, it is possible that the visual stimuli affect bias for males only holds for intent relevant stimuli (e.g. short robe). Likewise, hypothesis H2A failed to replicate in the intent irrelevant upper body exposure condition of study S2. Furthermore, the same effect was observed for females in all high object recognition conditions (of studies S2, S4A, S4B) while being non-significant (or absent) in all high intent conditions (studies S1B to S4B). Such concords with general hypotheses 1 and 2 , yet it suggests there are intent independent affective processes at work also (based simply on object recognition).

$\mathrm{H} 2 \mathrm{C}$ was fully supported by both manipulation conditions of S4A and S4B.

\section{General Discussion}

The results from the first two studies (S1-S2) are interpreted as supporting the theory (general hypotheses 1 and 2), however various alternative hypotheses should be considered.

With respect to general hypothesis 1, the study S1 (and S3) effect sizes are relatively weak and indicate that rational perception of intent is playing only a minor role in affective objectification. A much larger difference in self-report affect/arousal was observed between gender (for at least one exposed bodily region; the reproductive centre), than between the intent manipulation conditions of study S1. Likewise, study S1B indicated that this was not due to a confound with interpersonal affect.

Furthermore, study S4 (both S4A and S4B) failed to demonstrate that intent perception processes are more active than object recognition processes in affective objectification. The results from hypothesis H2B (across all studies) indicate that gender (male) specific affective objectification is only operative for lower body/reproductive system exposure stimuli (sexually suggestive short robe). Females were equally aroused by opposite sex exposed body shape (study S4) and complete upper body exposure (study S2) stimuli. Likewise, the results from hypothesis H2A (across all studies) indicate that gender (male) specific sensitivity to isolated opposite sex stimuli is only operative for lower body/reproductive system exposure stimuli (sexually suggestive short robe). The same effect was also observed for females with exposed body shape (study S4) and complete upper body exposure (study S2) stimuli. Although these findings are concordant with the general hypothesis (2), it suggests that stimulation tied to object recognition is also playing a role in affective objectification, and across both genders.

Perception of intent may therefore be a gender specific moderator rather than the core mechanism of affective objectification. Affective objectification could be a dual process, with innate perception of intent biases and innate object recognition biases both contributing to the affect. Supporting this interpretation, it is known that innate object recognition biases with respect to female sexual body parts (the inversion effect) are moderated by the provision of information concerning the target's internal warmth and competence [10]. Likewise, although discordant with the lack of generalisation of hypothesis H2B (and H2A) across stimuli (only lower body exposure, not high body shape exposure or upper body exposure; studies S2 and S4), innate affective gender biases in such object recognition processes should be considered (respecting the witness as opposed to the target gender, cf [9 11]).

Affective objectification might also be augmented by an attribution error (including the fundamental attribution error; [26]). Intention could be incorrectly inferred based on an extension of the self knowledge of one's natural (gender specific) affect in their presence to they themselves, the one responsible for that affect (inferring purpose rather than ignorance). The results from hypothesis $\mathrm{H} 1$ are not discordant with such a possibility, in that the neutral intent conditions tended to produce more affect than the 
negative intent conditions.

Moreover, affective objectification could at its core be an innate affective response (sexual arousal) to evolutionarily salient external stimuli (enacted by affectively biased object recognition processing systems). Any perception of intent moderation observed would thus be a product of intent-external stimuli conditioning; where an unconditioned (precoded) external stimulus (e.g. human body image) has intent stimuli associated with it (e.g. promiscuity), producing a conditioned response (where past experiences of promiscuity/intent were followed by bodily exposure). The influence of intent perception confirmed by this research would be an artefact of learnt associations or attention shifting (rather than being indicative of the underlying psychological mechanism).

Assuming that perception of intent is indeed driving an innate response for males however, it is possible that the attire chosen (short robe) for study S1 (across all manipulation conditions) triggered a subconscious perception of intent to be an object which was much stronger than any impression that could be conveyed to the contrary with words (i.e. that perception of intent was being predominately inferred by the visual as opposed to the verbal content). Likewise in study S4, intent perception could have been automatically triggered by the presence of opposite sex body shape (by either innate associations or conditioned associations with tight clothing), and automatic unconscious completion of partially occluded objects (short robe stimuli) cannot be ruled out. Again however, the lack of generalisation of hypothesis H2B (and H2A) across stimuli suggest that there are also intent (to be a sexually accessible object) independent object recognition processes at work. Females were equally affected by opposite sex stimuli with upper body exposure (study S2; non-reproductive system exposure) and high body shape exposure (study S4; tight pants).

Furthermore, if perception of intent is driving affective objectification for males (irrespective of the presence of an intent independent affective bias in object recognition), then the results (hypothesis $\mathrm{H} 1$ and $\mathrm{H} 2 \mathrm{~B}$; study $\mathrm{S} 1$ ) suggest that it must primarily be an irrational (subconscious) mechanism based on external stimuli-intent associations. (This should be distinguished from the intent-external stimuli conditioning discussed above, in which affective objectification is an object recognition response at its core). Such external stimuli-intent associations could either be innate or conditioned. Examples of conditioned external (visual) stimuli-intent associations include traditional societal interpretations of modesty, and pornography exposure (enhanced by the evolutionary salience of the experience; illusory copulation). In such cases, past experiences of bodily exposure are assumed here to have been followed by promiscuity/intent (producing a conditioned response to the exposed object). Contrarily, innate external stimuli-intent associations would involve a hard coded (evolved) association between the detection (observation) of exposed body parts and a subconscious perception of intent to reproduce (be an object).

It is questionable however whether such would be an adaptive response for a male organism, because it might trigger excessive conflict/competition, particularly when bodily exposure was the social norm (it is unlikely that clothing including its particular variations would be coded for given that it is a phylogenetically modern phenomenon). Yet it is difficult to conceive of any other stimuli which would reliably facilitate copulation for males if not externally derived (visual/tactile), and a precoded object recognition affective response (that does not trigger intent perception) would likely produce the same tensions in a preclothed community.

Critically however, it is theoretically questionable whether an innate unconscious external stimuli-intent affective association could be distinguished from a simple object recognition affective response (i.e. why would intent have to be coded for when the stimuli ultimately produce the same affective response). Under this model, affective objectification may not be operating on perception of intent per se, but on the 
perception of pre-coded stimuli indicative of relations, wherein much of this stimuli just so happened to signify intent in the environment of evolutionary adaptedness. One would therefore expect visual stimuli (e.g. positional) to cause arousal, even if the stimuli shown in their present context has nothing to do with relations. Such a model appears to conflict with affect moderation due to conscious processing of stimuli however, as weakly demonstrated by study S1 hypothesis H1.

In any case, intent recognition would have to confer some advantage on the organism's affective system, beyond that of modality independence (which could be accounted for by an object recognition system relying on multiple cues; visual/tactile). Intent recognition might for example prevent socially disadvantageous/disagreeable matings, when intent is expressed to the contrary of default (innate) object recognition based inferences (adaptationist moral psychology; [27]). Alternatively, and as here argued, it might encourage mating when it is more likely (or whenever it is likely) to be evolutionarily successful. For a male, this is simply when it is agreeable by the female (augmented by her reproductive health/fertility). For a female, this is when it is advantageous/optimum, in that her potential offspring would be expected to have a socially acceptable/dominant protector as opposed to being fathered by an opportunist/predator who is unable to gain access to females except but for short periods of time. Such explains not only the modality but the duration of stimulation experienced by females (in addition to the incentive the stimulation provides for engaging in natural reproductively consequential relations).

The successful (but weak) manipulations of hypothesis H1, and the lack of generalisation of hypothesis $\mathrm{H} 2 \mathrm{~B}$ and $\mathrm{H} 2 \mathrm{~A}$ across stimuli suggest the presence of such an intent dependent affective objectification process for males (as opposed to the activation of a simple affectively biased object recognition system). This research is however unable to distinguish between conditioned and hard coded stimuli-intent associations. Likewise, as discussed, this research is unable to rule out a prospective dual process (affectively biased intent perception and affectively biased object recognition), or intent-external stimuli conditioning (where affective objectification is an object recognition response at its core). In any such case, the possibility of unconscious intent associations remains difficult to test.

Affective objectification could also relate to perception of availability rather than intent. For example, if someone is situated such that it is physically possible for them to engage (/be engaged) in sexual relations, rather than it having anything to do with them encouraging such relations. Perception of intent does however have an explanatory advantage in that it generalises to/interacts with non-sexual objectification, unlike perception of availability. While it is theoretically meaningless (evolutionarily irrelevant) to talk about perception of availability in a non-sexual context, if the affect linked with objectification is caused by perception of intent to be/have an object, it is possible that the same affective processes might be triggered through alternate circumstances that are equally evolutionarily salient but non-sexual (perception of intent to dehumanise, dominate, disrespect, etc). Perception of availability is also more limited in explaining female affect in an evolutionary context (for a polygynous species). This research is however unable to distinguish between the effects of perception of intent and perception of availability.

Furthermore, the small effect observed in study S1 could be caused by or confounded by verbal priming (rather than being the product of intent attribution). That is, through the temporary modification of the primacy of one's goals, in that opposite sex interactions gain a higher importance. A priming effect would be unconnected to the specific visual stimuli, unlike in a successful perception of intent manipulation. The results (trends) from hypothesis $\mathrm{H} 1$ do however suggest that a priming explanation is unlikely (in that the neutral conditions tended to produce more affect than the negative 
verbal manipulation conditions; i.e. despite the absence of a verbal sexual prime). Likewise, the manipulation condition which would be expected to exhibit the greatest verbal sexual prime (M5/exotic dancer) consistently failed to demonstrate an overall positive manipulation. In study S1A, it demonstrated the lowest effect of all manipulations, and in study S1B it demonstrated less effect than the control condition. The results rather are concordant with a perception of intent theory, in that the sensual affect generated by a female visual stimulus is differentially biased based on expectations.

With respect to general hypothesis 2 , it is possible that the affective process is the same for males and females, and that the difference in effect observed between males and females is just an artefact of the male reproductive system being generally so well hidden. Study S2 offered some evidence for this alternate hypothesis (in that male and female affect primarily differed based on their response to the more dominant female reproductive system).

Likewise, hypotheses H2A and H2B appeared stimuli dependent. Males were only more attracted to opposite sex stimuli (H2B) for intent (to be a sexually accessible object) relevant stimuli (i.e. short robe, not tight pants or upper body exposure; studies S2 and S4). Similarly, females (not only males, as predicted by H2A) tended to be significantly more aroused by isolated opposite sex stimuli than male with female stimuli, for the intent irrelevant stimuli (i.e. tight pants and upper body exposure; studies S2 and S4). Furthermore, hypothesis H2A failed for males in study S2 (opposite sex upper body exposure). These observations provide evidence for the relevance of intent perception in male affective objectification. However, contrary to the theory, they suggest that an innate object recognition based affective response is also driving objectification (and in both males and females)-intent perception may be a subsidiary or dual process.

Alternatively, the presentation of the male body/reproductive system may signify the capacity to objectify (supporting the beauty/handsome distinction inherited by the west). If it cannot signify such a capacity, then according to the (single process) theory (3), it would have no influence on female affect. Such speculation is supported by differences in sexual motivation inherent in evolutionary theory.

Yet the theory is also supported by natural reproductive system exposure differences during intercourse (penetration and pose; in at least, the environment of evolutionary adaptedness). If these exposure differences are emphasised, then the theory can be framed more broadly: that males and females are both sensitive to natural stimuli experienced during intercourse, but a significant part of that stimuli for females is not visible (visual). From this perspective, it would be more valid to identify the sexual object as the point of contact within the female reproductive system (rather than the system itself).

Contrarily, females might even be affected by disinterested males, given that in the environment of evolutionary adaptedness, disinterest could signify dominance (the availability/surplus of genetically higher quality females). If this were the case, then there would be no complementary form of intent dependent affective objectification for females. Similarly, affective objectification may have had minimal adaptive advantage for females, as per their environment of evolutionary adaptiveness (for a typical sexually dimorphic species in which males compete for access to females).

An investigation of general hypothesis 3 is required to disentangle these interpretations. Furthermore, such an investigation must test target (directional) specific indicators of desire to have an object (e.g. tactile, verbal, etc), which are theorised to be critical in conveying such intent for females. 


\section{Limitations}

A significant limitation of this research is that only self-reported affect data were measured. It is possible that participants (males or females) were over or under representing their affect on the likert scales. Presentation bias could have affected the hypothesis $\mathrm{H} 2 \mathrm{~A}$ result in particular (lowering the affect generated by a male with female stimulus). Likewise, although self-report arousal was assumed to better capture affective objectification than the self-report sensual affect measure using in study S1A (with respect to the confounding of interpersonal affect), it still may not have properly captured the construct. For example, it could have captured non-sexual arousal to an extent (and again its interpretation might have varied across gender). The general findings should be replicated with physiological measurements of arousal.

Moreover, the stimuli were not reverse ordered. This was not considered a problem considering that the stimuli were all presented on the same page, the intent manipulations should not be affected by the order of the stimuli (1), and males and females should not be differentially affected by the order of the stimuli $(\mathrm{H} 2 \mathrm{~B} / \mathrm{H} 2 \mathrm{C})$. Again however, hypothesis H2A is most risked by this limitation. Similarly, the individual and paired stimuli were not assigned to separate participants (conditions). Yet again this could have affected the hypothesis H2A result (exacerbating any presentation bias).

Participants could also have been affected by the normality of the stimuli. As short robes (and robes in general) are not common apparel for males in contemporary western culture, they could have been interpreted as unusual (weird), which may have decreased female affect/arousal. Likewise, males could have been conditioned to interpret the female short robe stimuli as indicative of intent to be an object, whereas such conditioning may not have existed for females with respect to the male short robe stimuli. The same hypothetical anomalies could have influenced responses to the female stimulus without head hair (study S3). Although any modification in affect (separate from the visual stimuli itself) supports the general theory (1), it may have adversely influenced the hypothesis $\mathrm{H} 2 \mathrm{~B}$ result (males being more attracted to female visual stimuli than vice versa).

It is difficult however to find sexualised clothing that controls for both perceived normality and bodily shape, without inadvertently manipulating perception of intent (given innate differences in reproductive system dominance). The only normative sexualised clothing that controls for bodily shape equally across females and males is tight (form fit) clothing, but this overemphasises the female reproductive system, and may therefore inadvertently bias perception of intent across stimulus gender (unlike the non-normative robe stimuli). Moreover, tight clothing, although capable in principle of triggering a prospective innate affective bias in human object recognition, is not necessarily sexual suggestive (indicative of sexual accessibility). It may in fact convey information to the contrary (as was assumed to be the case in study S4).

Likewise, participants could have been differentially affected by the male/female stimuli based on their stances. The males adopted a wider stance and the females adopted a narrower stance. Only hypotheses $\mathrm{H} 2 \mathrm{~B} / \mathrm{H} 2 \mathrm{C}$ and $\mathrm{H} 3 \mathrm{~A} / \mathrm{H} 3 \mathrm{~B}$ are risked by this limitation however, as these compared the affect generated by male and female stimuli.

Furthermore, these findings are not immune to stimuli conditioning and sexual activity biases across gender, and should therefore be replicated cross-culturally. Male participants could for example be conditioned to viewing more exposed female stimuli (due to the prevalence of pornography), or be more likely to be in a sexually sedated (or primed) state. Such would affect the ability to measure perception of intent and infer innate response differences across gender. Hypotheses $\mathrm{H} 2 \mathrm{~B}, \mathrm{H} 3$, and $\mathrm{H} 5$ are most risked by this limitation.

Extending on study S4 (hypothesis H5), the two theories of affective objectification 
(intent versus innate object recognition bias) might be further disentangled by presenting participants with images containing either low body exposure but high intent (e.g. completely dressed/null lower body shape exposure with desirous facial expression) or high body exposure but low intent (e.g. nakedness in a natural non-sexual context). This design would not however discriminate between the two theories if (a lack of) body exposure had already been associated with (a lack of) intent (as it might neutralise the intent manipulation).

Finally, the affect (stimulation) measured may not relate to cognitive objectification, or have any behavioural consequences (such is unlikely but requires testing). Likewise, it might not represent an innate adaptive response to the detection of certain parameters in the environment (perception of intent or object recognition). The affect experienced in objectification manipulations could be a byproduct of a (socialised) cognitive assessment, a conditioned response based purely on prior sexual experience, or even an intentional response (e.g. the desire to please, revenge, etc). These all however fail to address how affect/arousal would be generated within actual sexual experiences (i.e. the nature of the subconscious adaptive response that facilitates copulation).

\section{Implications}

As sexualisation is becoming increasingly normative in western civilisation (at least in its media), it is important to have a solid theoretical framework for predicting its implications on a person's psychology. Objectification theory from its conception has provided an interpretative framework for modern society [28], including both explanations of change and restrictions to freedom. This work presents one possible mechanism behind the phenomenon of affective objectification, and it should be critiqued against other upcoming candidates. Furthermore, it should help to illuminate cognitive and behavioural objectification.

\section{Conclusion}

The underling mechanism of objectification was investigated. It was theorised that affective objectification is the adaptive subconscious perception of intent to be or have an object (for males and females respectively). Manipulating the perception of intent of a female within a visual stimulus weakly modified self-report sensual affect/arousal ratings for males. Likewise, the affect generated by a female stimulus was influenced by the absence of a male partner, and males were more affected by opposite sex visual stimuli than females (for intent dependent stimuli). This work is however unable to test target specific indicators of desire to have an object, and further research is required to test general hypothesis 3. Moreover, the results suggest that perception of intent may be a gender specific moderator rather than the sole cause of affective objectification. Innate affective biases in object recognition should be considered.

\section{Supporting Information}

\section{S1 Appendix}

Objectification interpretations in the empirical literature

\section{S2 Appendix}

\section{Surveys}




\section{S3 Appendix}

\section{Bad data filtering}

\section{References}

1. Vaes J, Paladino P, Puvia E. Are sexualized women complete human beings? Why men and women dehumanize sexually objectified women. European Journal of Social Psychology. 2011;41(6):774-785.

2. Nussbaum MC. Objectification. Philosophy \& Public Affairs. 1995;24(4):249-291.

3. Bartky SL. Femininity and domination: Studies in the phenomenology of oppression. Psychology Press; 1990.

4. Holland E. From somebodies to bodies: towards a better understanding of the causes, process, and consequences of sexual objectification; 2013.

5. Morris KL, Goldenberg JL. Women, objects, and animals: Differentiating between sex-and beauty-based objectification. Revue internationale de psychologie sociale. 2015;28(1):15-38.

6. Heflick NA, Goldenberg JL, Cooper DP, Puvia E. From women to objects: Appearance focus, target gender, and perceptions of warmth, morality and competence. Journal of Experimental Social Psychology. 2011;47(3):572-581.

7. Rudman LA, Mescher K. Of animals and objects men's implicit dehumanization of women and likelihood of sexual aggression. Personality and Social Psychology Bulletin. 2012;38(6):734-746.

8. Puvia E, Vaes J. Being a body: Women's appearance related self-views and their dehumanization of sexually objectified female targets. Sex Roles. 2013;68(7-8):484-495.

9. Bernard P, Gervais SJ, Allen J, Campomizzi S, Klein O. Integrating Sexual Objectification With Object Versus Person Recognition The

Sexualized-Body-Inversion Hypothesis. Psychological Science. 2012;23(5):469-471.

10. Bernard P, Gervais SJ, Allen J, Delmée A, Klein O. From Sex Objects to Human Beings Masking Sexual Body Parts and Humanization as Moderators to Women's Objectification. Psychology of women quarterly. 2015;39(4):432-446.

11. Gervais SJ, Vescio TK, Förster J, Maass A, Suitner C. Seeing women as objects: The sexual body part recognition bias. European Journal of Social Psychology. 2012;42(6):743-753.

12. Moor A. She Dresses to Attract, He Perceives Seduction: A gender gap in attribution of intent to women's revealing style of dress and its relation to blaming the victims of sexual violence. Journal of International Women's Studies. 2010;11(4):115.

13. Gervais SJ, Bernard P, Riemer AR. Who treats people as sex objects? Cultural orientation, social comparison, and sexual objectification perpetration. Revue internationale de psychologie sociale. 2015;28(1):153-181.

14. Morris KL, Goldenberg JL, Heflick NA. Trio of terror (pregnancy, menstruation, and breastfeeding): An existential function of literal self-objectification among women. Journal of personality and social psychology. 2014;107(1):181. 
15. Gray K, Knobe J, Sheskin M, Bloom P, Barrett LF. More than a body: mind perception and the nature of objectification. Journal of personality and social psychology. 2011;101(6):1207.

16. Cikara M, Eberhardt JL, Fiske ST. From agents to objects: Sexist attitudes and neural responses to sexualized targets. Journal of Cognitive Neuroscience. $2011 ; 23(3): 540-551$.

17. Loughnan S, Haslam N, Murnane T, Vaes J, Reynolds C, Suitner C. Objectification leads to depersonalization: The denial of mind and moral concern to objectified others. European Journal of Social Psychology. 2010;40(5):709-717.

18. Fox J, Bailenson JN. Virtual virgins and vamps: The effects of exposure to female characters' sexualized appearance and gaze in an immersive virtual environment. Sex roles. 2009;61(3-4):147-157.

19. Murnen SK. Gender and the use of sexually degrading language. Psychology of Women Quarterly. 2000;24(4):319-327.

20. Gervais SJ, Holland AM, Dodd MD. My eyes are up here: The nature of the objectifying gaze toward women. Sex Roles. 2013;69(11-12):557-570.

21. Dijkstra P, Buunk BP. Sex differences in the jealousy-evoking nature of a rival's body build. Evolution and Human Behavior. 2001;22(5):335-341.

22. Jiang Y, Costello P, Fang F, Huang M, He S. A gender-and sexual orientation-dependent spatial attentional effect of invisible images. Proceedings of the National Academy of Sciences. 2006;103(45):17048-17052.

23. Schmidt G. Male-female differences in sexual arousal and behavior during and after exposure to sexually explicit stimuli. Archives of Sexual Behavior;4(4):353-365.

24. Hinsz VB, Matz DC, Patience RA. Does women's hair signal reproductive potential? Journal of Experimental Social Psychology. 2001;37(2):166-172.

25. Swami V, Furnham A, Joshi K. The influence of skin tone, hair length, and hair colour on ratings of women's physical attractiveness, health and fertility. Scandinavian Journal of Psychology. 2008;49(5):429-437.

26. Ross L. The intuitive psychologist and his shortcomings: Distortions in the attribution process. Advances in experimental social psychology. 1977;10:173-220.

27. Haidt J. The emotional dog and its rational tail: A social intuitionist approach to moral judgment. Psychological review. 2001 Oct;108(4):814-834.

28. Fredrickson BL, Roberts TA. Objectification theory. Psychology of women quarterly. 1997;21(2):173-206. 


\section{S1 Appendix - Objectification interpretations in the empirical literature}

Objectification is typically portrayed in terms of behavioural, cognitive, or affective phenomena [1].

Objectification has been associated with the behaviour of the objectified person [1], the person (witness) processing them as an object [1, or the person responsible for their presentation.

Objectification has been considered to occur when a target's attributes or behaviour are modified, for example clothing type [2]5], facial dominance [3, 4], eye gaze [6], or presentation with sexually degrading language [7.

Likewise, objectification has been considered to occur when a witness's behaviours or general thought patterns are modified. Relevant modifications to general cognition include the tendency to view women as sexual objects or the maintenance of behaviourally inappropriate attitudes, both of which may be induced by an experience of sexualised media 8. Likewise, people have been considered to objectify a target based on how they subsequently relate to a female [9-12]. Accordingly, objectification has assumed to have been invoked when they are encouraged to think about a person in terms of their appearance rather than their character [3, 13]. Evidence suggests that this affects perceptions of warmth, competence, and morality [14.

Furthermore, objectification is often associated with the process by which a person is presented by a third party. Objectification can thus be seen as a retrospective cognitive judgement (classification) of behaviour, independent of the behaviour, cognition, or affect of the objectified person or witness.

Other researchers see objectification as a specific cognitive process in the witness with regards to the target [1].

Many have measured trait objectification consciousness [15 18 using modifications of the Fredrickson et al. [19] Self-Objectification Questionnaire (SOQ). Such ask participants to rank order appearance based attributes with regards to other people's bodies. Others have measured specific interpretations of the target, with respect to both sexualisation [5, 20, 21] and dehumanisation [1, 3, [5, 13, 22. Still others have sexually primed participants, and then queried whether they would wish to work with an incompetent but attractive female [5, 23]. Finally, some have measured objectification as the extent to which targets are seen as fungible or interchangeable with like others [24].

Objectification has also been associated with innate differences in the cognitive processing of sexualised bodies (object recognition). Persons in general are more difficult to recognise upside down, but this inversion effect is reduced for sexualised female bodies [25]. Bernard et al. 26] suggests that this effect is specific to female sexual body parts, and is moderated by the provision of information concerning the target's internal warmth and competence. Likewise, objectification has been defined based on the way in which humans process body parts. Evidence suggests that female body parts (like objects) are processed locally (in isolation), whereas male body parts are processed globally (in context) [27.

Finally, objectification has been associated with modifications in affect. It is often implicitly associated with modifications in affect (e.g. attraction, desirability) [20, 21]. Furthermore, self-report arousal has been measured with respect to the presentation of female visual stimuli [5].

\section{References}

1. Holland E. From somebodies to bodies: towards a better understanding of the causes, process, and consequences of sexual objectification; 2013. 
2. Cikara M, Eberhardt JL, Fiske ST. From agents to objects: Sexist attitudes and neural responses to sexualized targets. Journal of Cognitive Neuroscience. 2011;23(3):540-551.

3. Gray K, Knobe J, Sheskin M, Bloom P, Barrett LF. More than a body: mind perception and the nature of objectification. Journal of personality and social psychology. 2011;101(6):1207.

4. Loughnan S, Haslam N, Murnane T, Vaes J, Reynolds C, Suitner C. Objectification leads to depersonalization: The denial of mind and moral concern to objectified others. European Journal of Social Psychology. 2010;40(5):709-717.

5. Vaes J, Paladino P, Puvia E. Are sexualized women complete human beings? Why men and women dehumanize sexually objectified women. European Journal of Social Psychology. 2011;41(6):774-785.

6. Fox J, Bailenson JN. Virtual virgins and vamps: The effects of exposure to female characters' sexualized appearance and gaze in an immersive virtual environment. Sex roles. 2009;61(3-4):147-157.

7. Murnen SK. Gender and the use of sexually degrading language. Psychology of Women Quarterly. 2000;24(4):319-327.

8. Yao MZ, Mahood C, Linz D. Sexual priming, gender stereotyping, and likelihood to sexually harass: Examining the cognitive effects of playing a sexually-explicit video game. Sex roles. 2010;62(1-2):77-88.

9. Jansma LL, Linz DG, Mulac A, Imrich DJ. Men's interactions with women after viewing sexually explicit films: Does degradation make a difference?

Communications Monographs. 1997;64(1):1-24.

10. McKenzie-Mohr D, Zanna MP. Treating Women as Sexual Objects Look to the (Gender Schematic) Male Who has Viewed Pornography. Personality and Social Psychology Bulletin. 1990;16(2):296-308.

11. Mulac A, Jansma L, Linz D. Men's behavior toward women after viewing sexually-explicit films: Degradation makes a difference. Communication Monographs. 2002;69(4):311-328.

12. Rudman LA, Borgida E. The afterglow of construct accessibility: The behavioral consequences of priming men to view women as sexual objects. Journal of Experimental Social Psychology. 1995;31(6):493-517.

13. Heflick NA, Goldenberg JL. Objectifying Sarah Palin: Evidence that objectification causes women to be perceived as less competent and less fully human. Journal of Experimental Social Psychology. 2009;45(3):598-601.

14. Heflick NA, Goldenberg JL, Cooper DP, Puvia E. From women to objects: Appearance focus, target gender, and perceptions of warmth, morality and competence. Journal of Experimental Social Psychology. 2011;47(3):572-581.

15. Grabe S, Routledge C, Cook A, Andersen C, Arndt J. In defense of the body: The effect of mortality salience on female body objectification. Psychology of Women Quarterly. 2005;29(1):33-37.

16. Kozak M, Frankenhauser H, Roberts TA. Objects of desire: Objectification as a function of male sexual orientation. Psychology of Men \& Masculinity.

$2009 ; 10(3): 225$. 
17. Strelan P, Hargreaves D. Women who objectify other women: The vicious circle of objectification? Sex Roles. 2005;52(9-10):707-712.

18. Swami V, Coles R, Wilson E, Salem N, Wyrozumska K, Furnham A. Oppressive beliefs at play: Associations among beauty ideals and practices and individual differences in sexism, objectification of others, and media exposure. Psychology of Women Quarterly. 2010;34(3):365-379.

19. Fredrickson BL, Roberts TA, Noll SM, Quinn DM, Twenge JM. That swimsuit becomes you: sex differences in self-objectification, restrained eating, and math performance. Journal of personality and social psychology. 1998;75(1):269.

20. Gurung RA, Chrouser CJ. Predicting objectification: Do provocative clothing and observer characteristics matter? Sex Roles. 2007;57(1-2):91-99.

21. Johnson V, Gurung RA. Defusing the objectification of women by other women: The role of competence. Sex Roles. 2011;65(3-4):177-188.

22. Rudman LA, Mescher K. Of animals and objects men's implicit dehumanization of women and likelihood of sexual aggression. Personality and Social Psychology Bulletin. 2012;38(6):734-746.

23. Gruenfeld DH, Inesi ME, Magee JC, Galinsky AD. Power and the objectification of social targets. Journal of personality and social psychology. 2008;95(1):111.

24. Gervais SJ, Vescio TK, Allen J. When are people interchangeable sexual objects? The effect of gender and body type on sexual fungibility. British Journal of Social Psychology. 2012;51(4):499-513.

25. Bernard P, Gervais SJ, Allen J, Campomizzi S, Klein O. Integrating Sexual Objectification With Object Versus Person Recognition The Sexualized-Body-Inversion Hypothesis. Psychological Science. 2012;23(5):469-471.

26. Bernard P, Gervais SJ, Allen J, Delmée A, Klein O. From Sex Objects to Human Beings Masking Sexual Body Parts and Humanization as Moderators to Women's Objectification. Psychology of women quarterly. 2015;39(4):432-446.

27. Gervais SJ, Vescio TK, Förster J, Maass A, Suitner C. Seeing women as objects: The sexual body part recognition bias. European Journal of Social Psychology. 2012;42(6):743-753. 


\section{S2 Appendix - Surveys}

\section{Survey S1A (USA participants)}

Survey Title: "Affect Survey"

Instructions:

"Overview - This survey shows a series of images and asks you to rate the sensual effect they have on you.

Process - Read and answer the questions to the best of your ability.

Thank you! - Thank you for your honest answers in this task.

(Note this survey is used for scientific research. There are 5 questions in total, and it should take 1-2 minutes to complete carefully)." 


\section{Questions}

Ballet Dancer [M1] / Family Friend [M2] / no text [M3] / Nightclub Dancer [M4] / Exotic Dancer [M5]

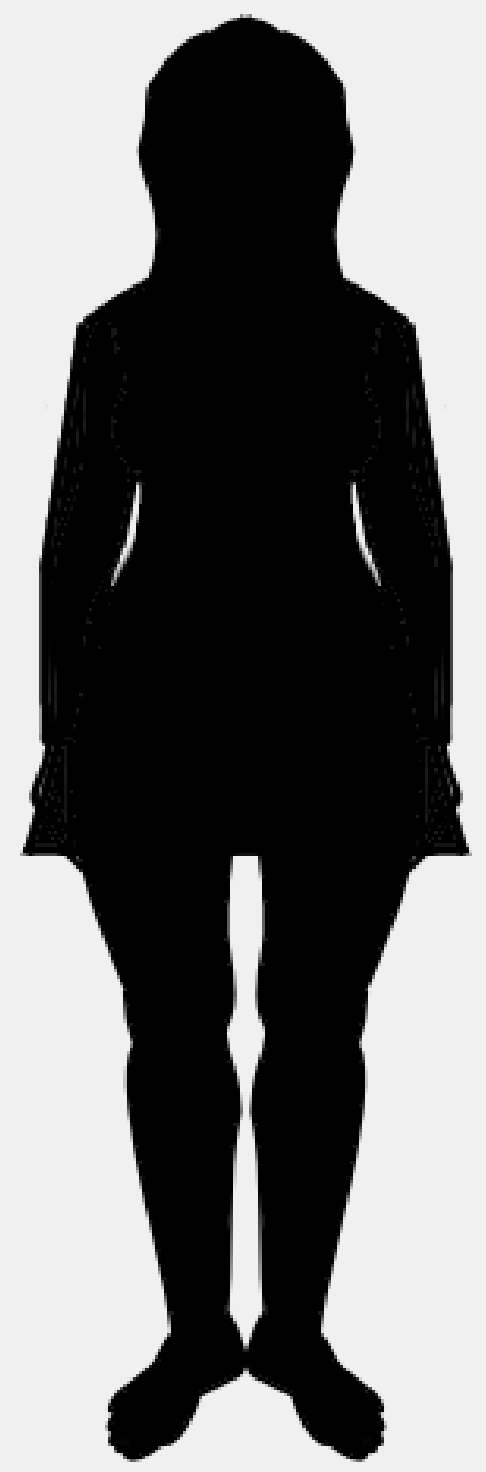

- How aroused are you by the above image? 
Ballet Dancer [M1] / Family Friend [M2] / no text [M3] / Nightclub Dancer [M4] / Exotic Dancer [M5]

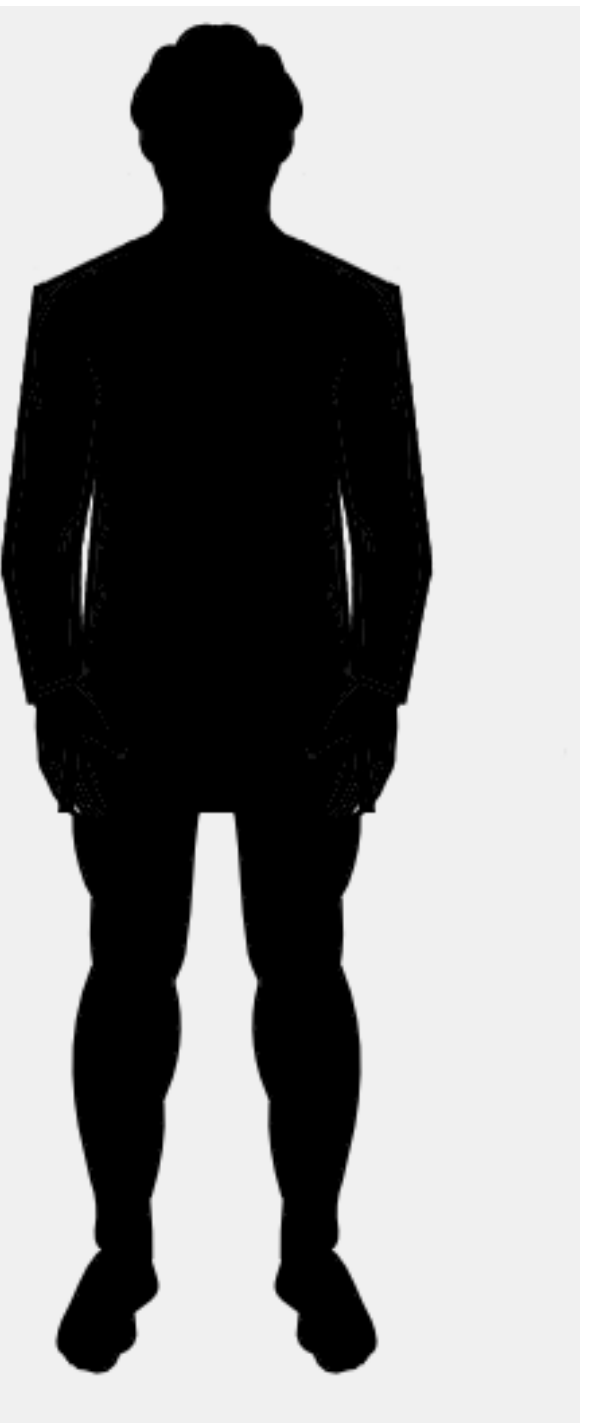

- How aroused are you by the above image? 
Ballet Dancers [M1] / Family Friends [M2] / no text [M3] / Nightclub Dancers [M4] / Exotic Dancers [M5]

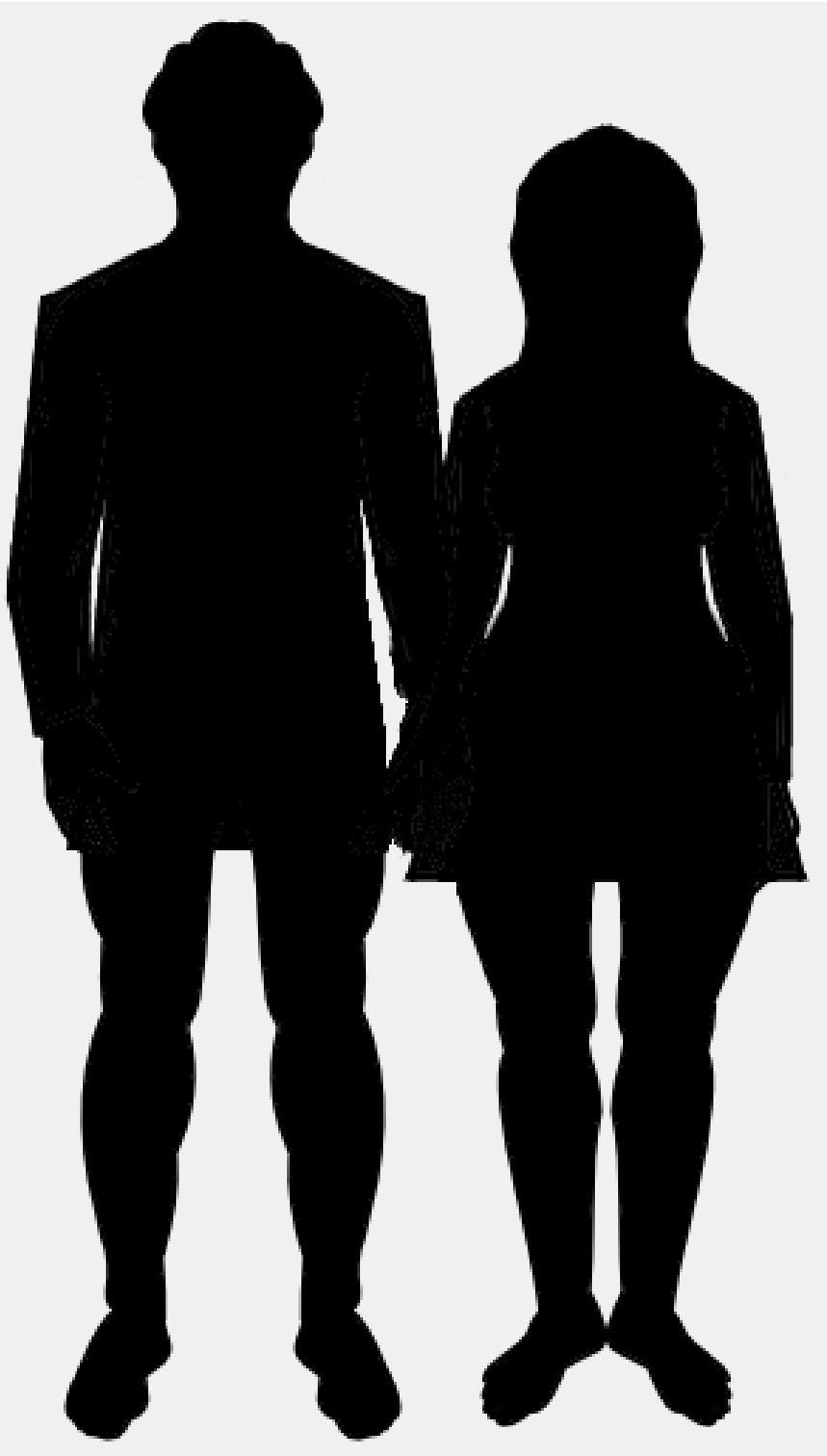

- How aroused are you by the above image? 
- How good is it for a person to rob a bank?

- Your Gender

Ten level Likert scale labels ('How sensually affected are you by the above image?')

- 1. not affected at all

- 10. extremely affected

Multiple Choice Options ('How good is it for a person to rob a bank?')

- somewhat good

- fairly good

- quite good

- very good

- extremely good

- not good at all

Multiple Choice Options (gender)

- male

- female 


\section{Survey S1B (USA participants)}

Survey Title: "Affect Survey"

Instructions:

"Overview - This survey shows a series of images and asks you to rate the level of arousal you experience.

Process - Read and answer the questions to the best of your ability.

Thank you! - Thank you for your honest answers in this task.

(Note this survey is used for scientific research. There are 5 questions in total, and it should take approximately 1 minute to complete carefully)." 


\section{Questions}

Ballet Dancer [M1] / Family Friend [M2] / no text [M3] / Nightclub Dancer [M4] / Exotic Dancer [M5]

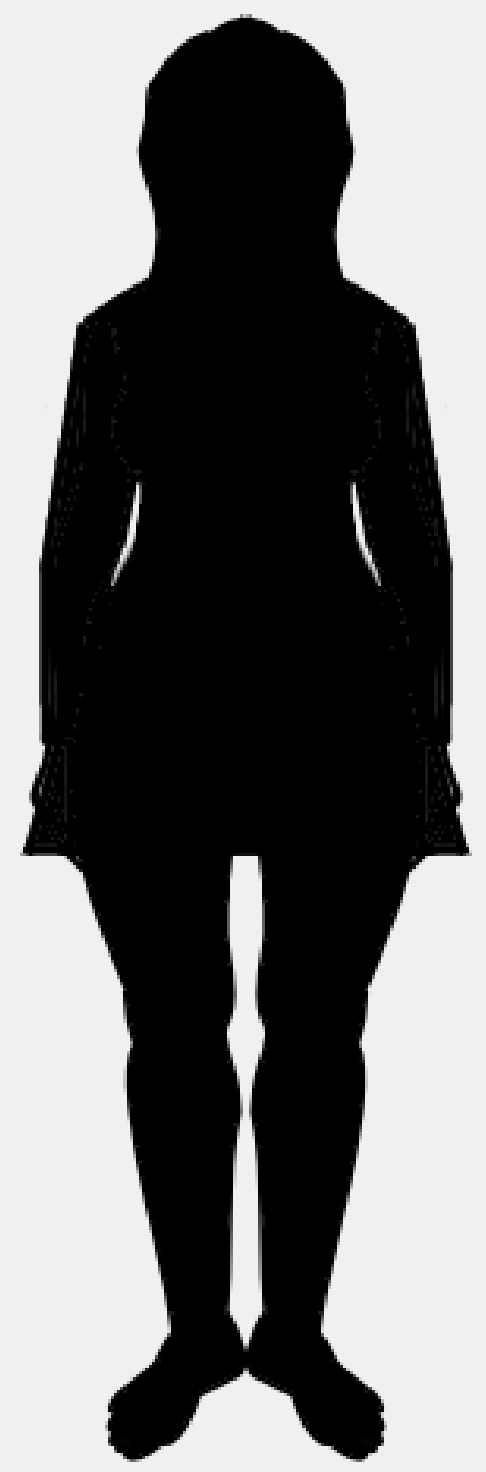

- How aroused are you by the above image? 
Ballet Dancer [M1] / Family Friend [M2] / no text [M3] / Nightclub Dancer [M4] / Exotic Dancer [M5]

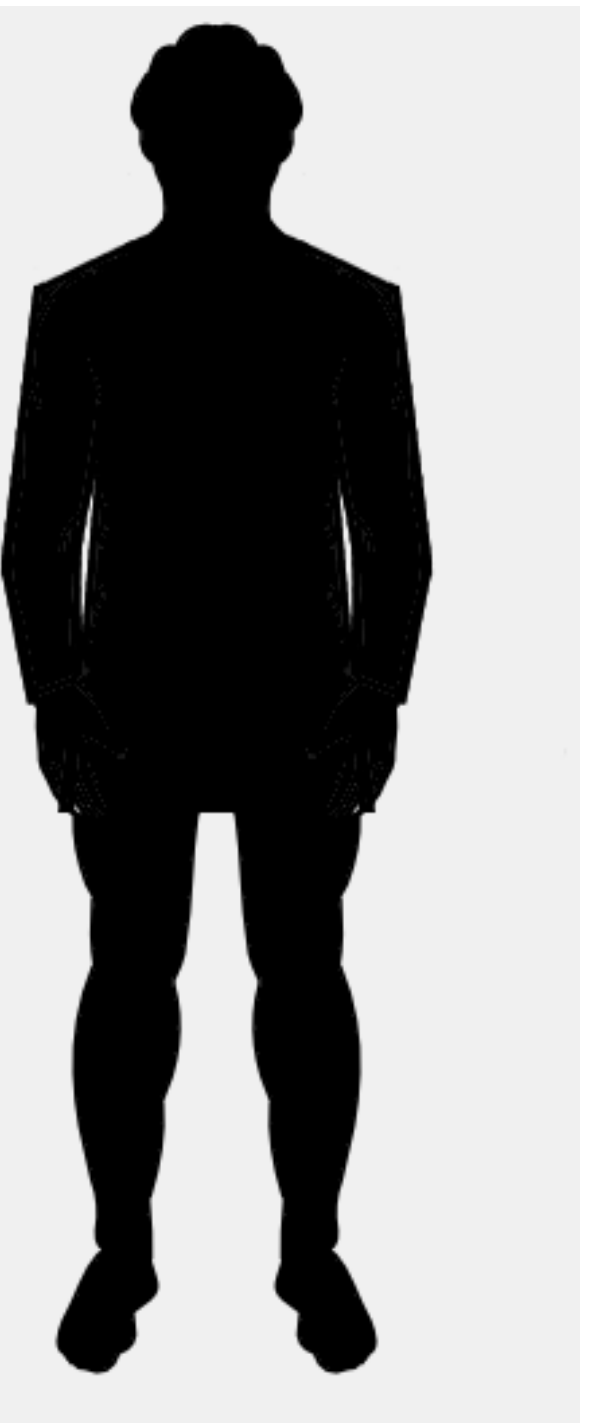

- How aroused are you by the above image? 
Ballet Dancers [M1] / Family Friends [M2] / no text [M3] / Nightclub Dancers [M4] / Exotic Dancers [M5]

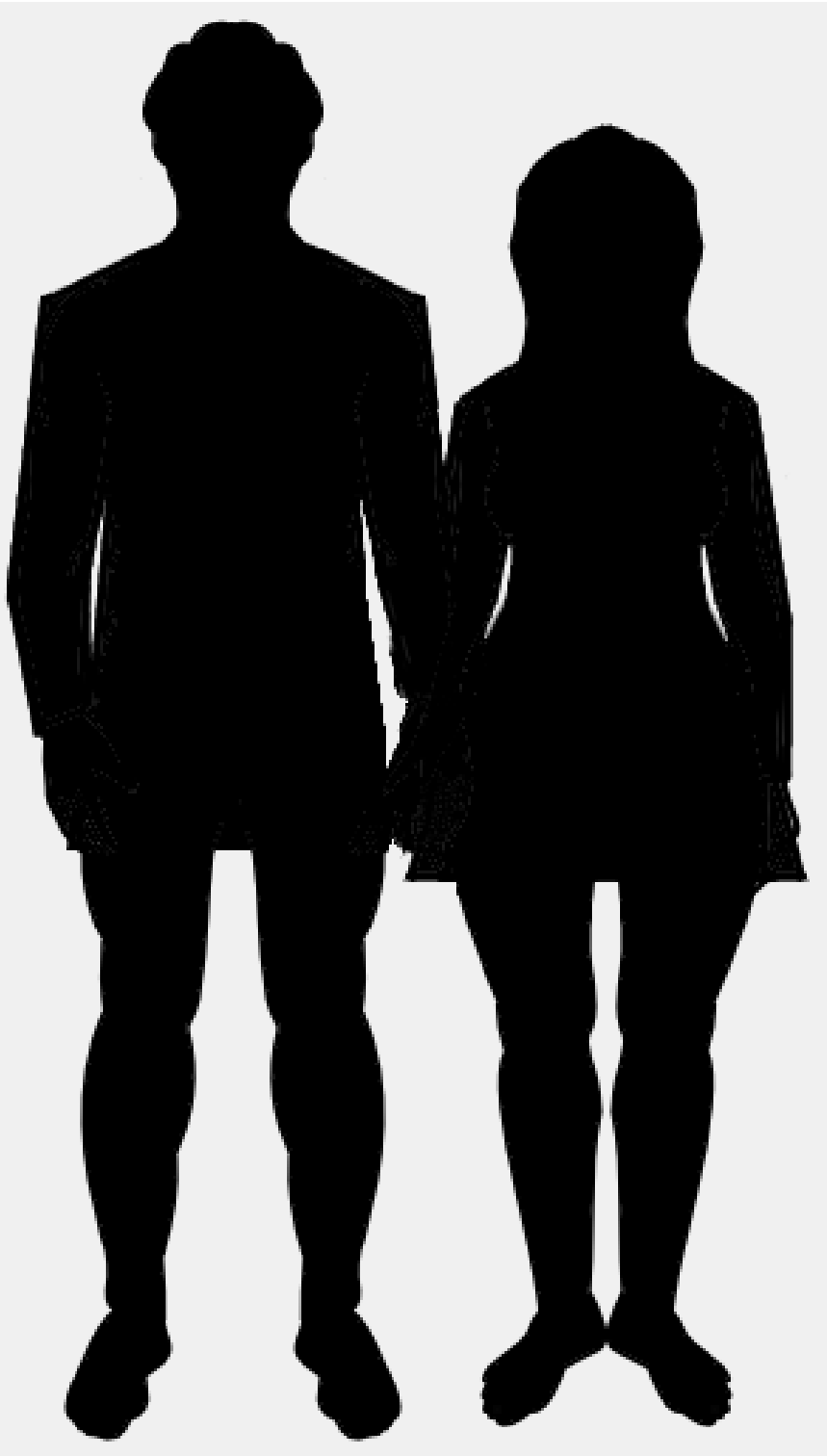

- How aroused are you by the above image? 
- How bad is it to help a friend?

- Your Gender

Ten level Likert scale labels ('How aroused are you by the above image?')

- 1. not aroused at all

- 10. extremely aroused

Multiple Choice Options ('How bad is it to help a friend?')

- somewhat bad

- not bad at all

- fairly bad

- quite bad

- very bad

- extremely bad

Multiple Choice Options (gender)

- male

- female 


\section{Survey S2 (USA participants)}

Survey Title: "Affect Survey"

Instructions:

"Overview - This survey shows a series of images and asks you to rate the level of arousal you experience.

Process - Read and answer the questions to the best of your ability.

Thank you! - Thank you for your honest answers in this task.

(Note this survey is used for scientific research. There are 5 questions in total, and it should take approximately 1 minute to complete carefully)." 


\section{Questions}

Manipulation 1 (upper body exposure):

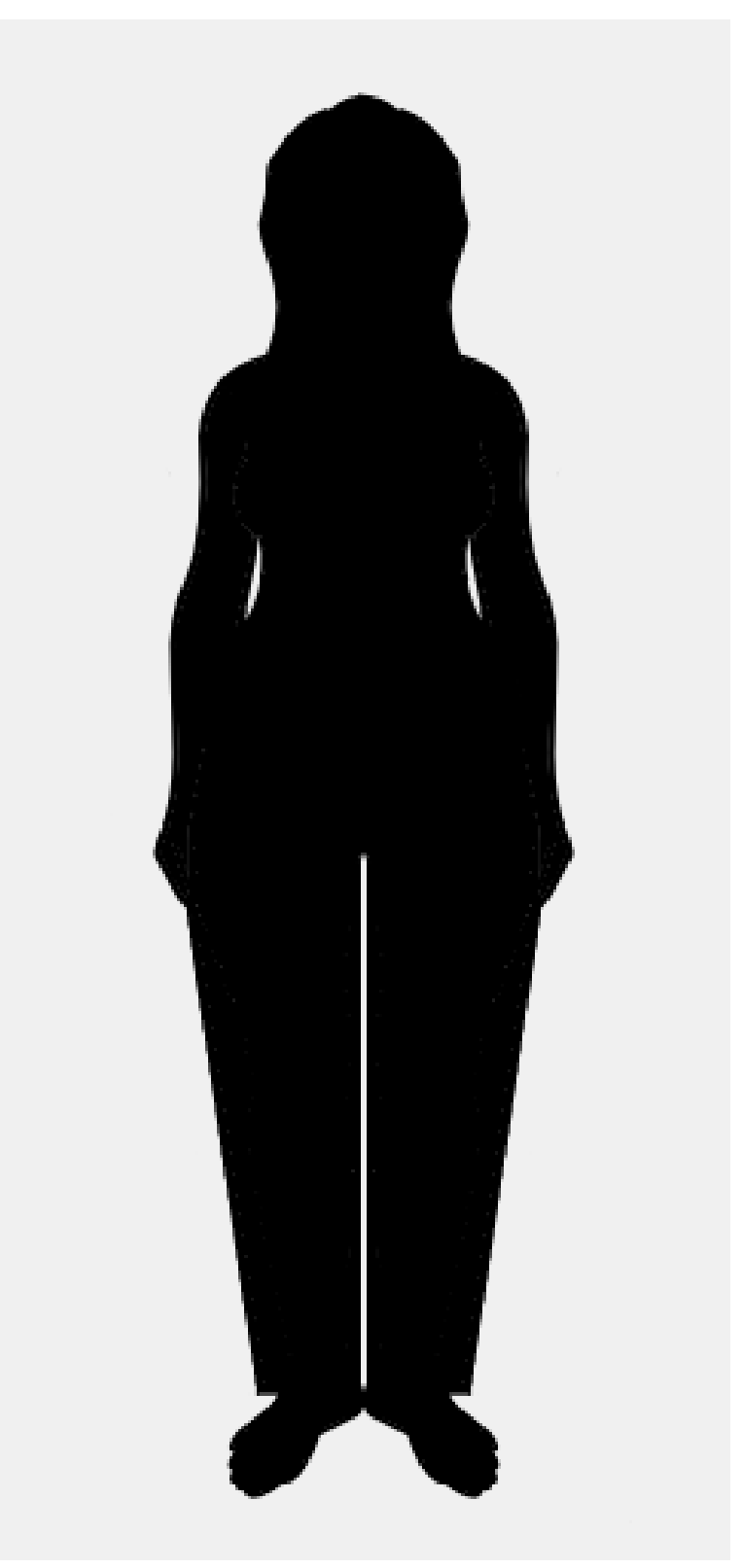

- How aroused are you by the above image? 


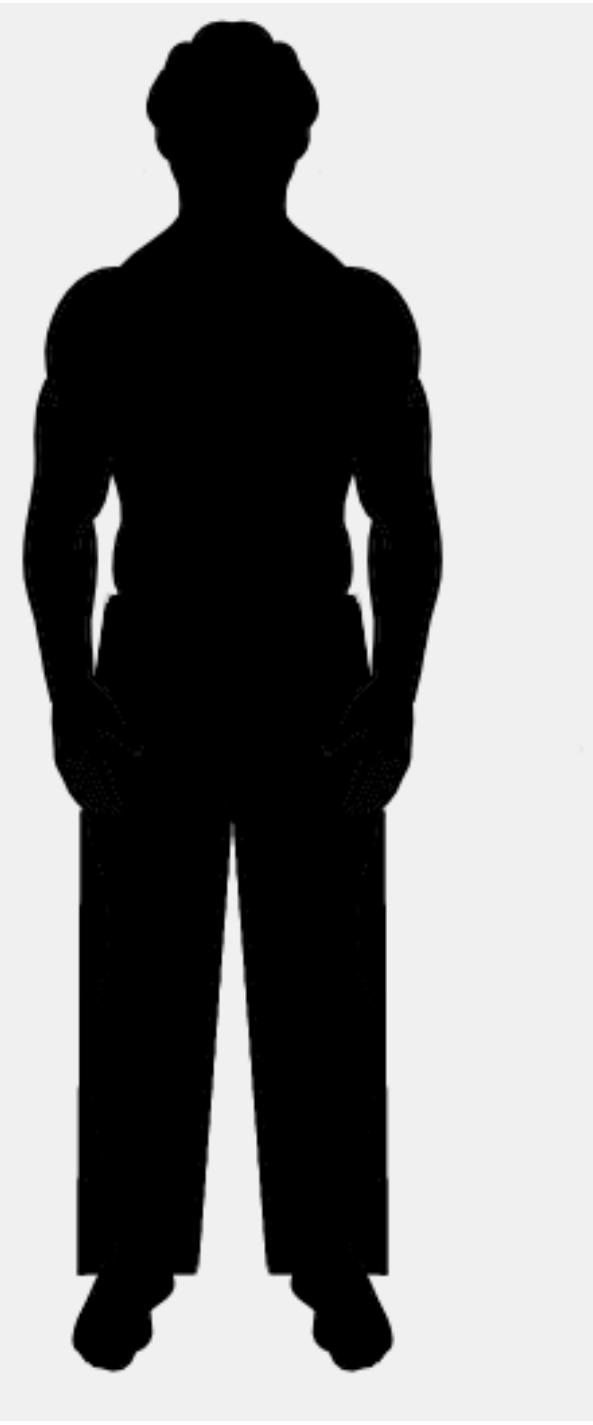

- How aroused are you by the above image? 


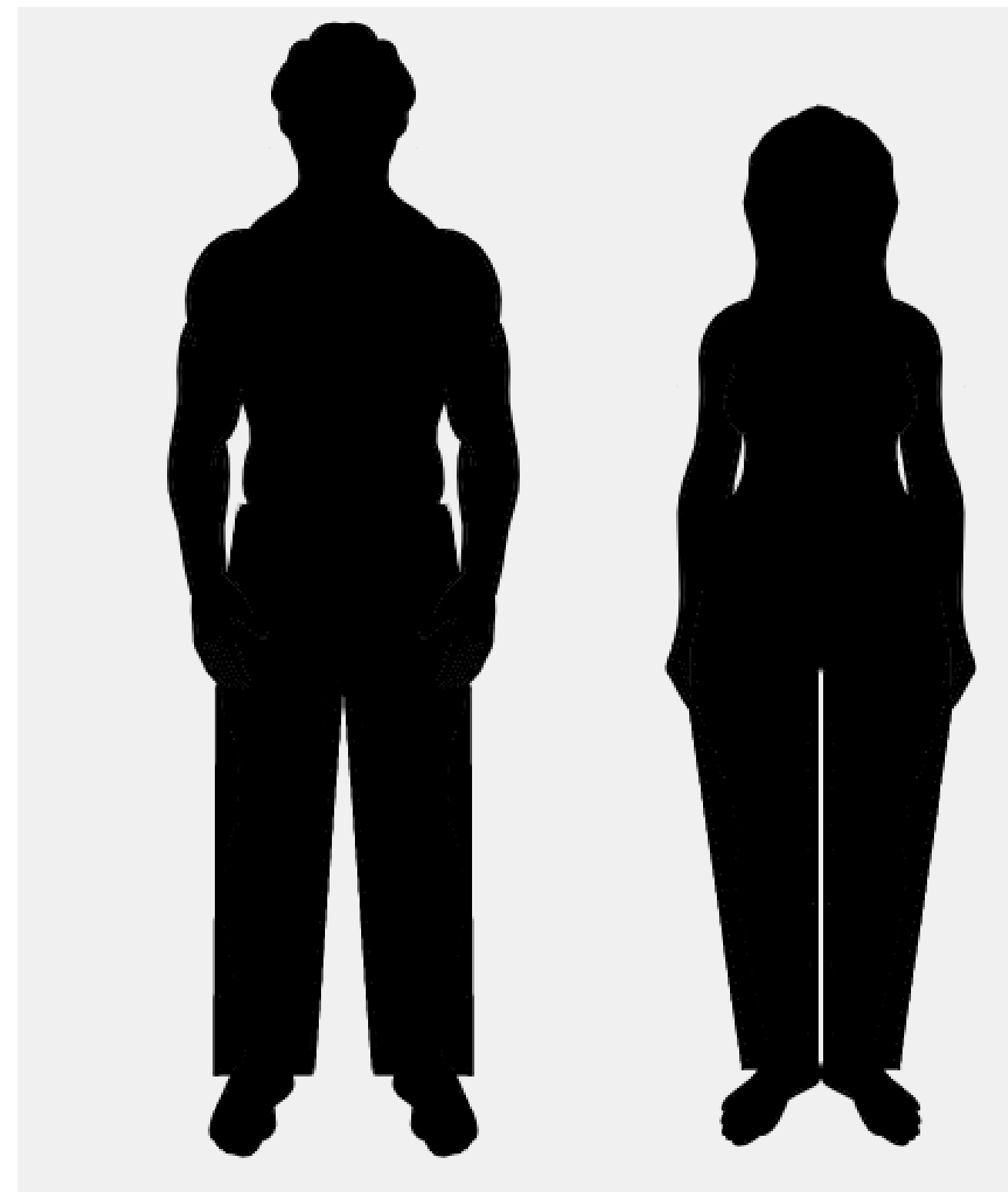

Manipulation 2 (short robe): 


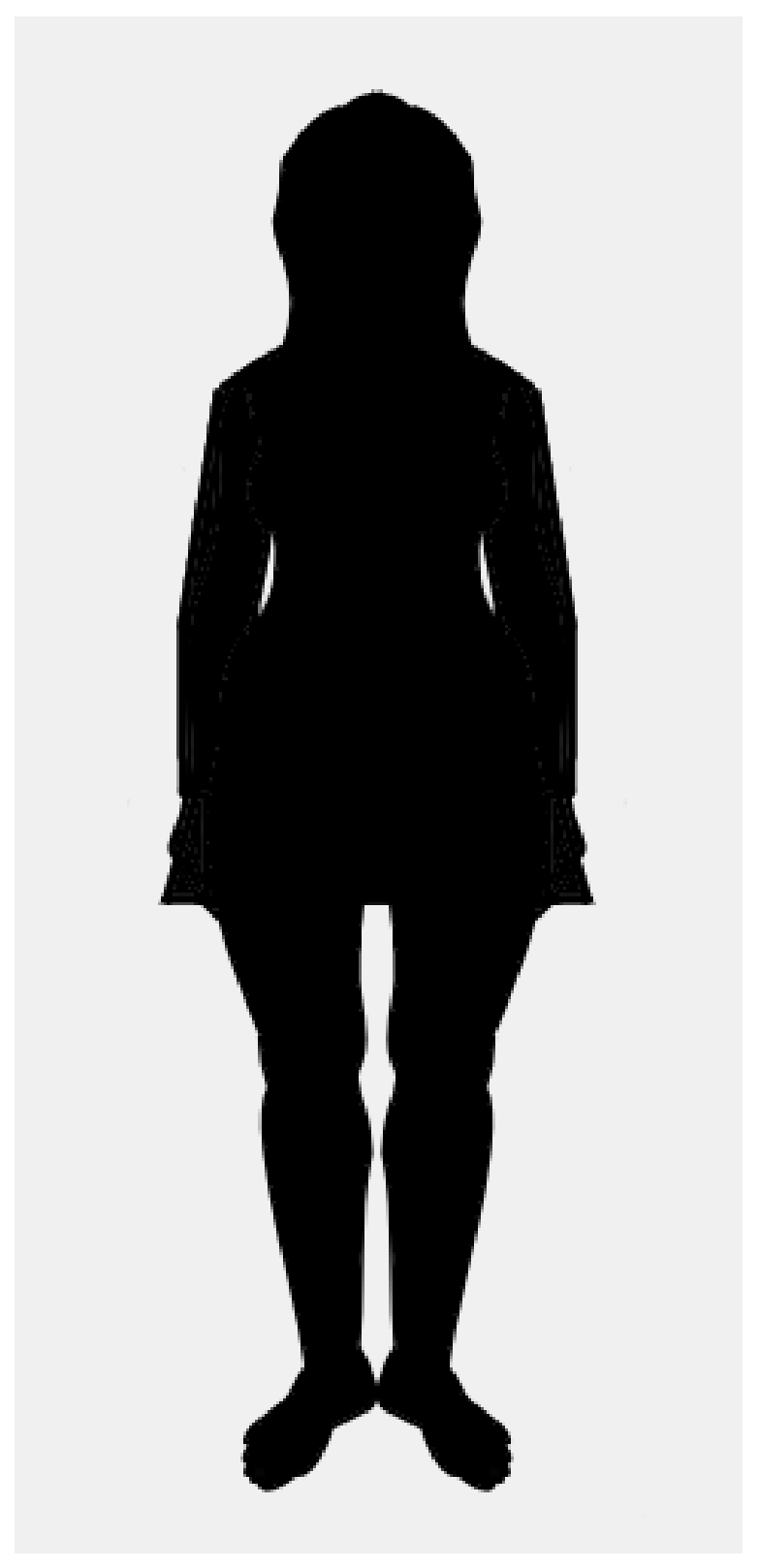

- How aroused are you by the above image? 


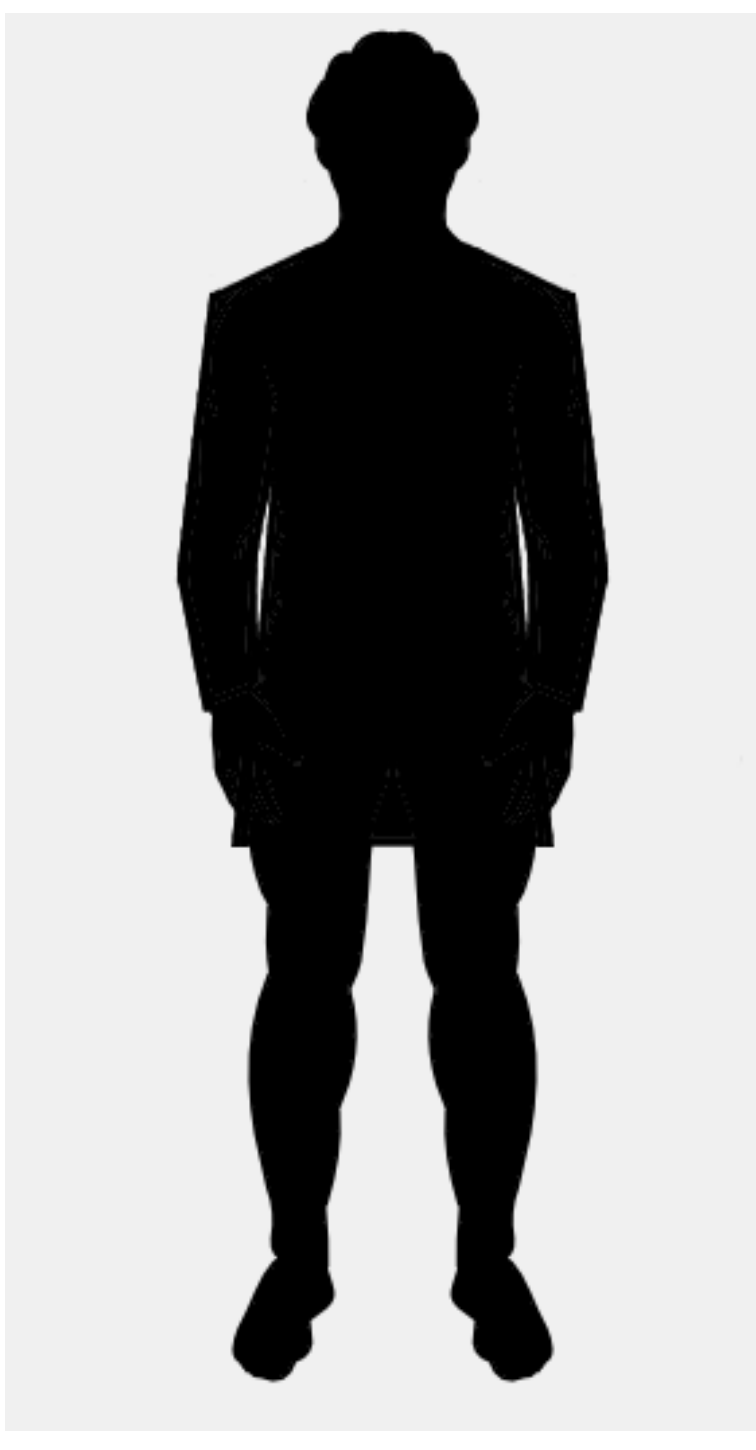

- How aroused are you by the above image? 


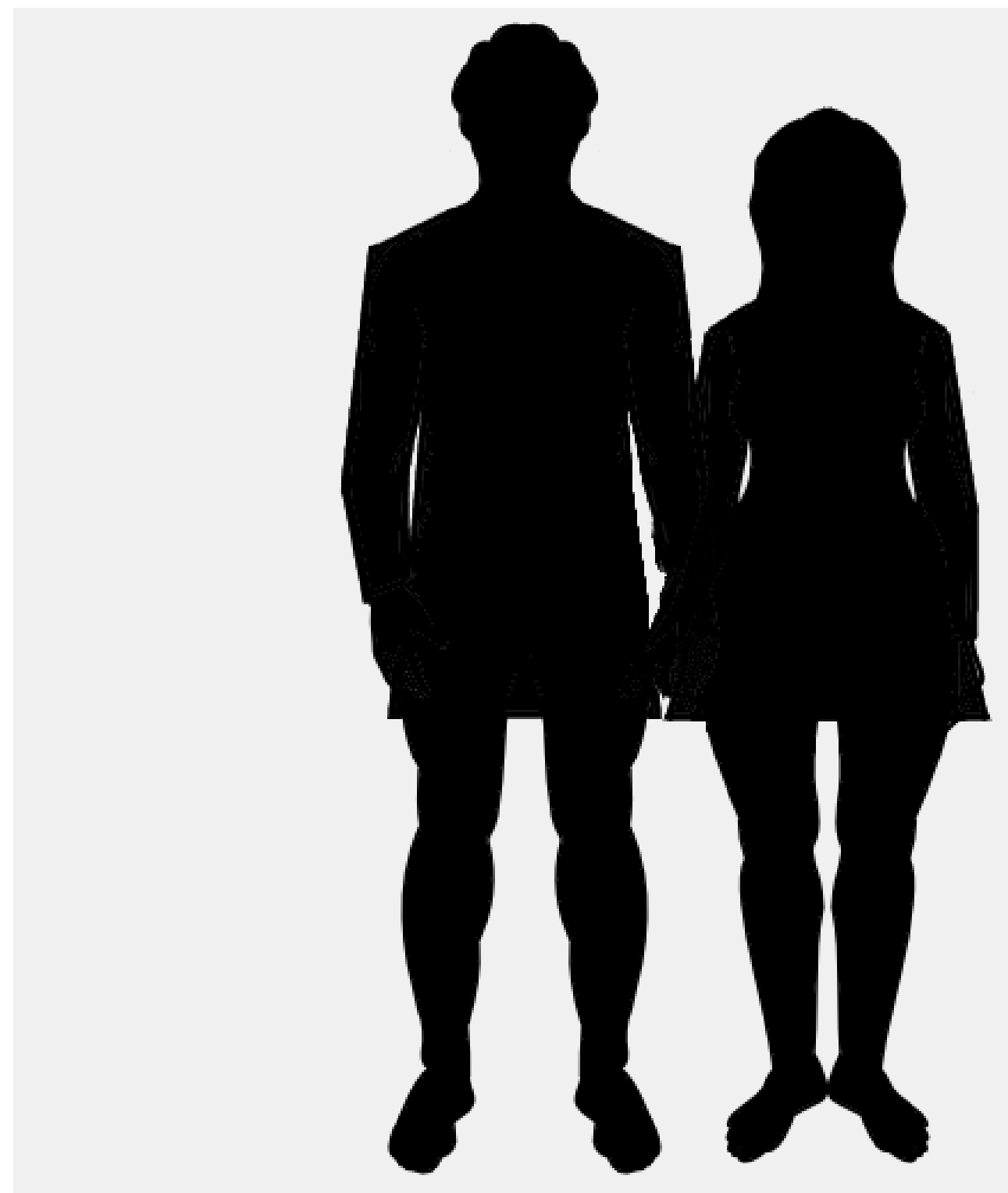

- How aroused are you by the above image? 
- How bad is it to help a friend?

- Your Gender

Ten level Likert scale labels ('How aroused are you by the above image?')

- 1. not aroused at all

- 10. extremely aroused

Multiple Choice Options ('How bad is it to help a friend?')

- somewhat bad

- fairly bad

- quite bad

- very bad

- not bad at all

- extremely bad

Multiple Choice Options (gender)

- male

- female 


\section{Survey S3 (USA participants)}

Survey Title: "Affect Survey"

Instructions:

"Overview - This survey shows a series of images and asks you to rate the level of arousal you experience.

Process - Read and answer the questions to the best of your ability.

Thank you! - Thank you for your honest answers in this task.

(Note this survey is used for scientific research. There are 5 questions in total, and it should take approximately 1 minute to complete carefully)." 


\section{Questions}

Manipulation 1 (no head hair):

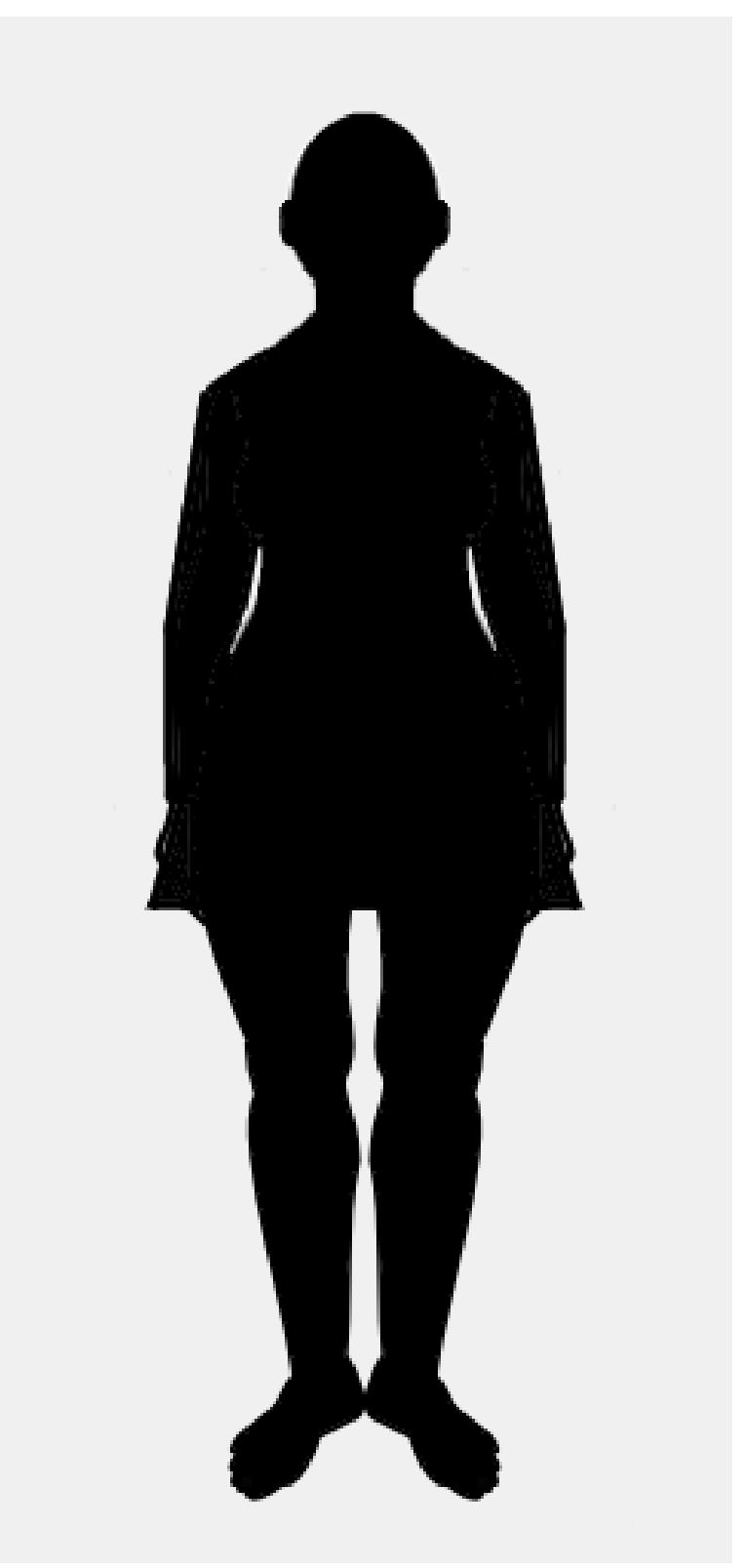

- How aroused are you by the above image? 


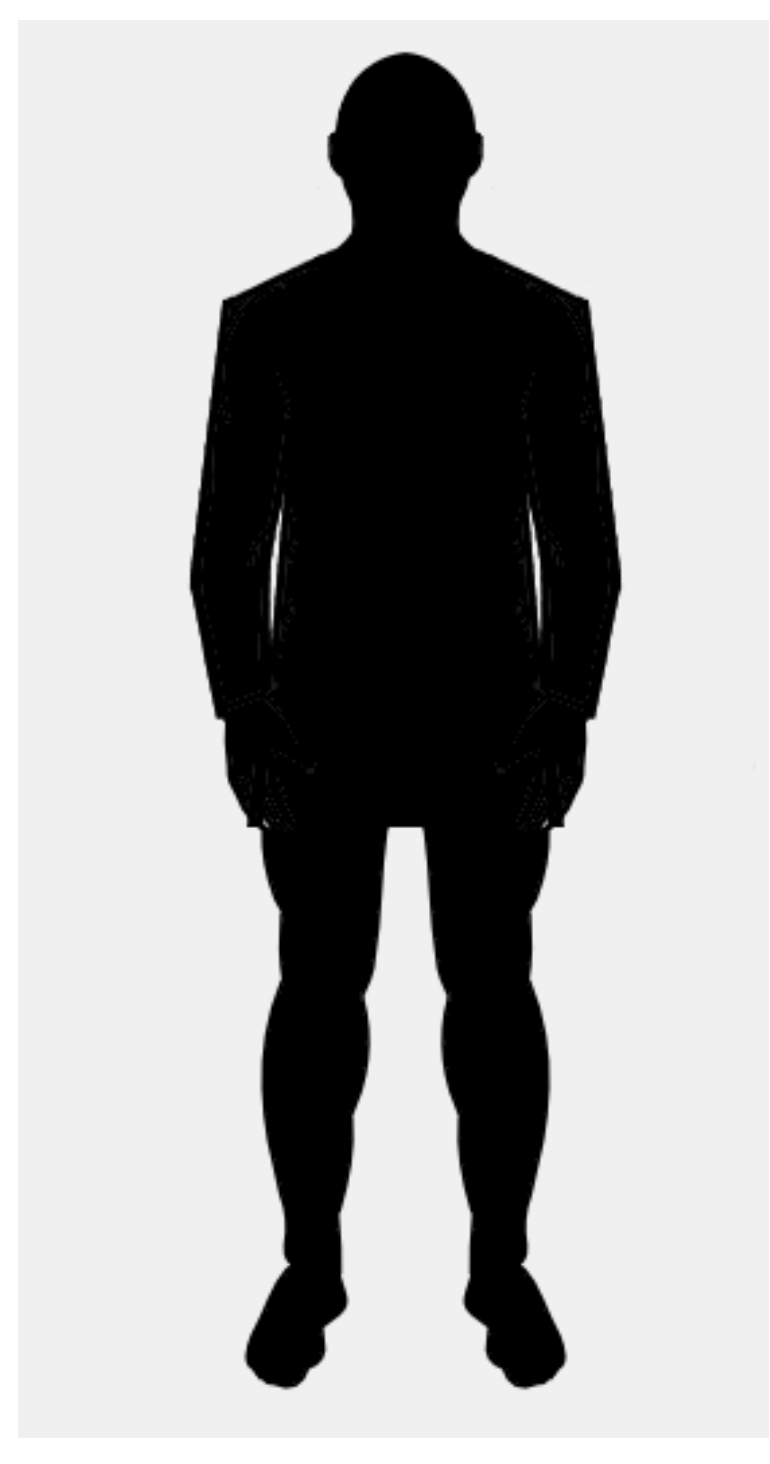

- How aroused are you by the above image? 


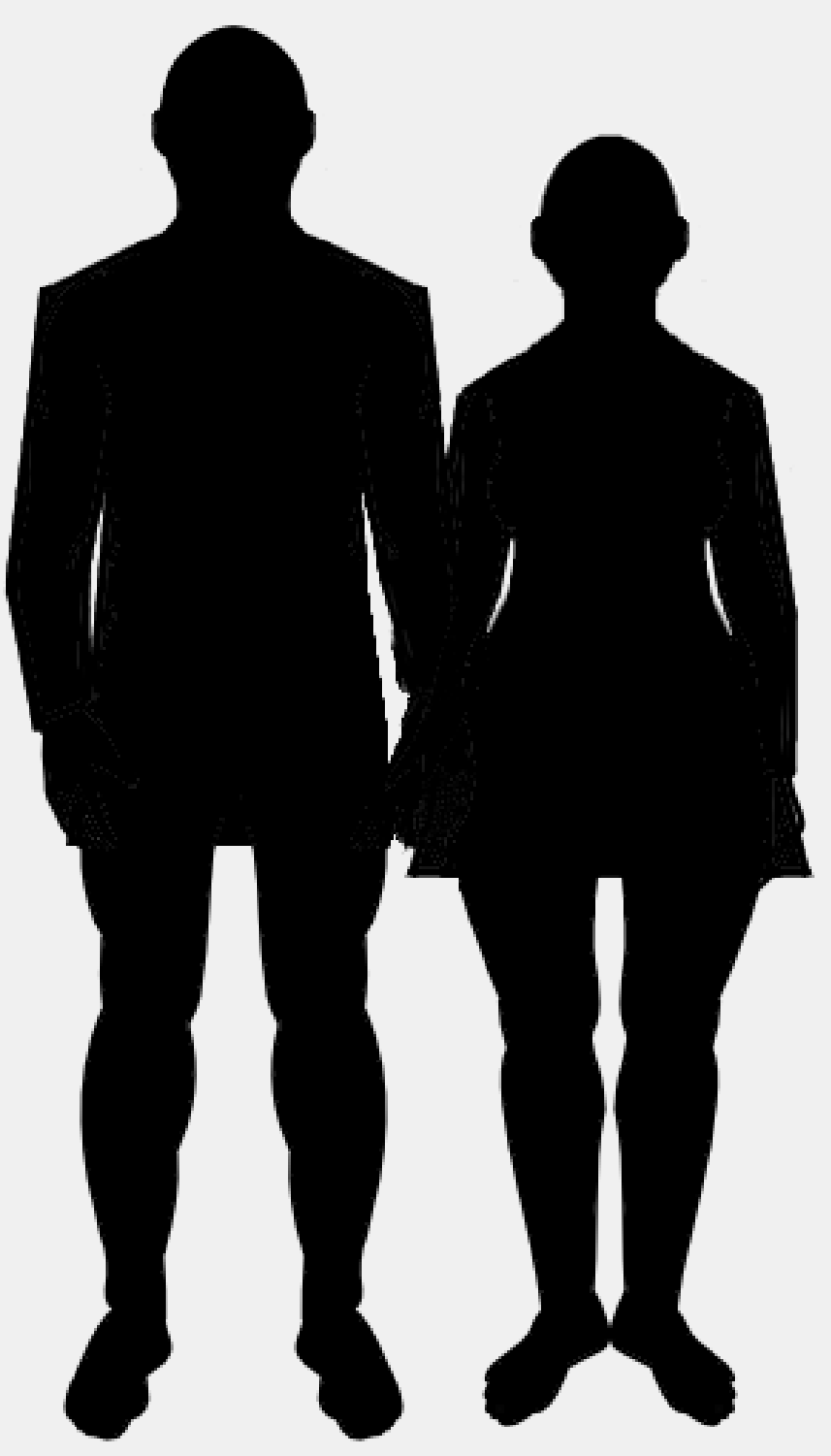

- How aroused are you by the above image? 
Manipulation 2 (short robe):

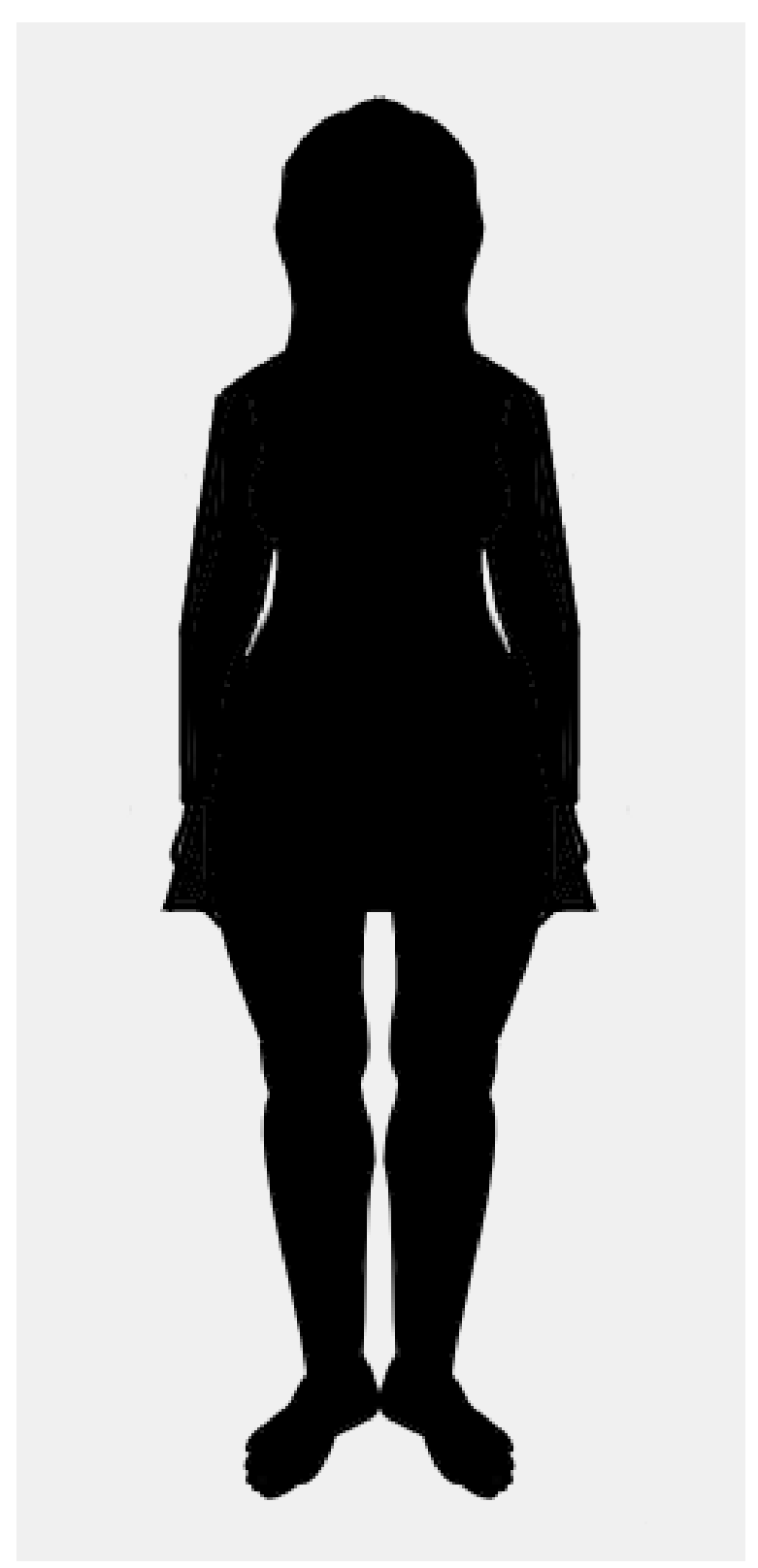

- How aroused are you by the above image? 


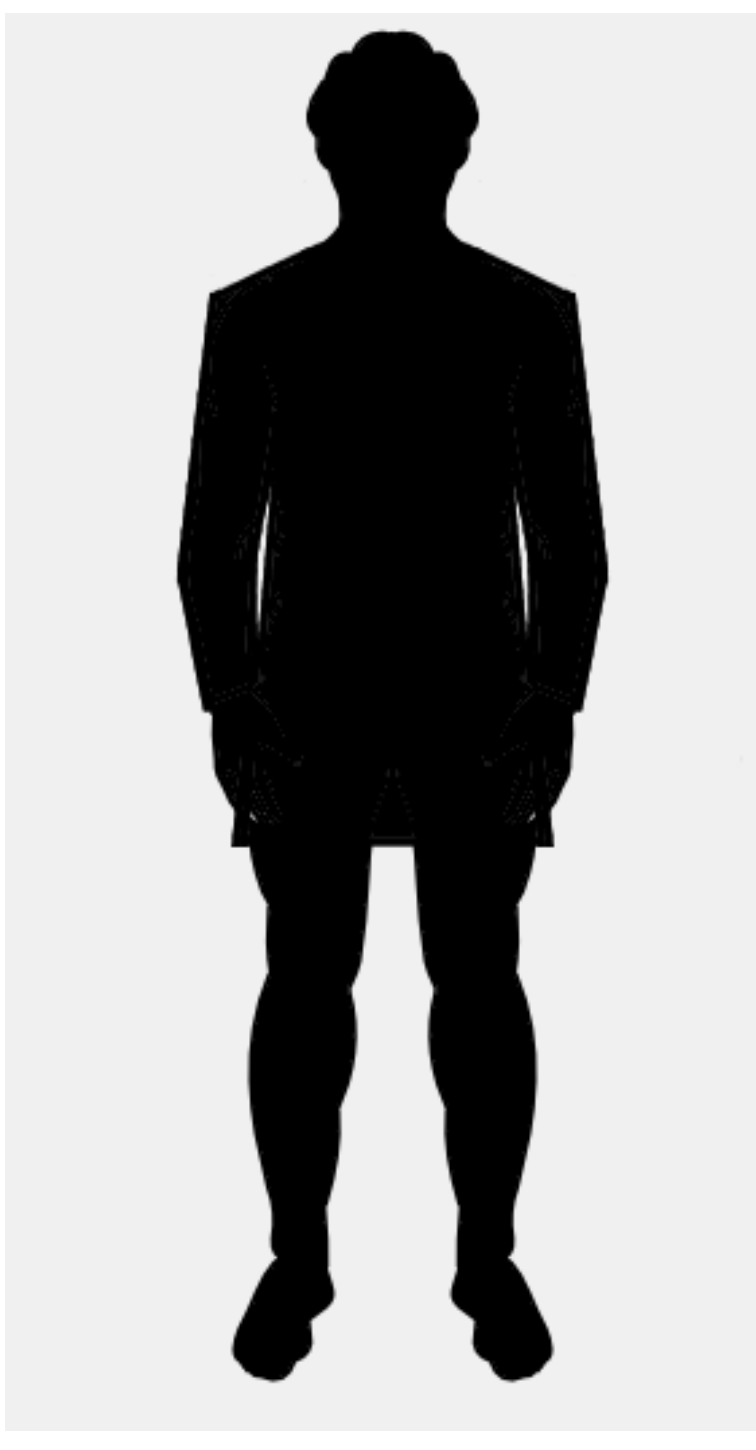

- How aroused are you by the above image? 


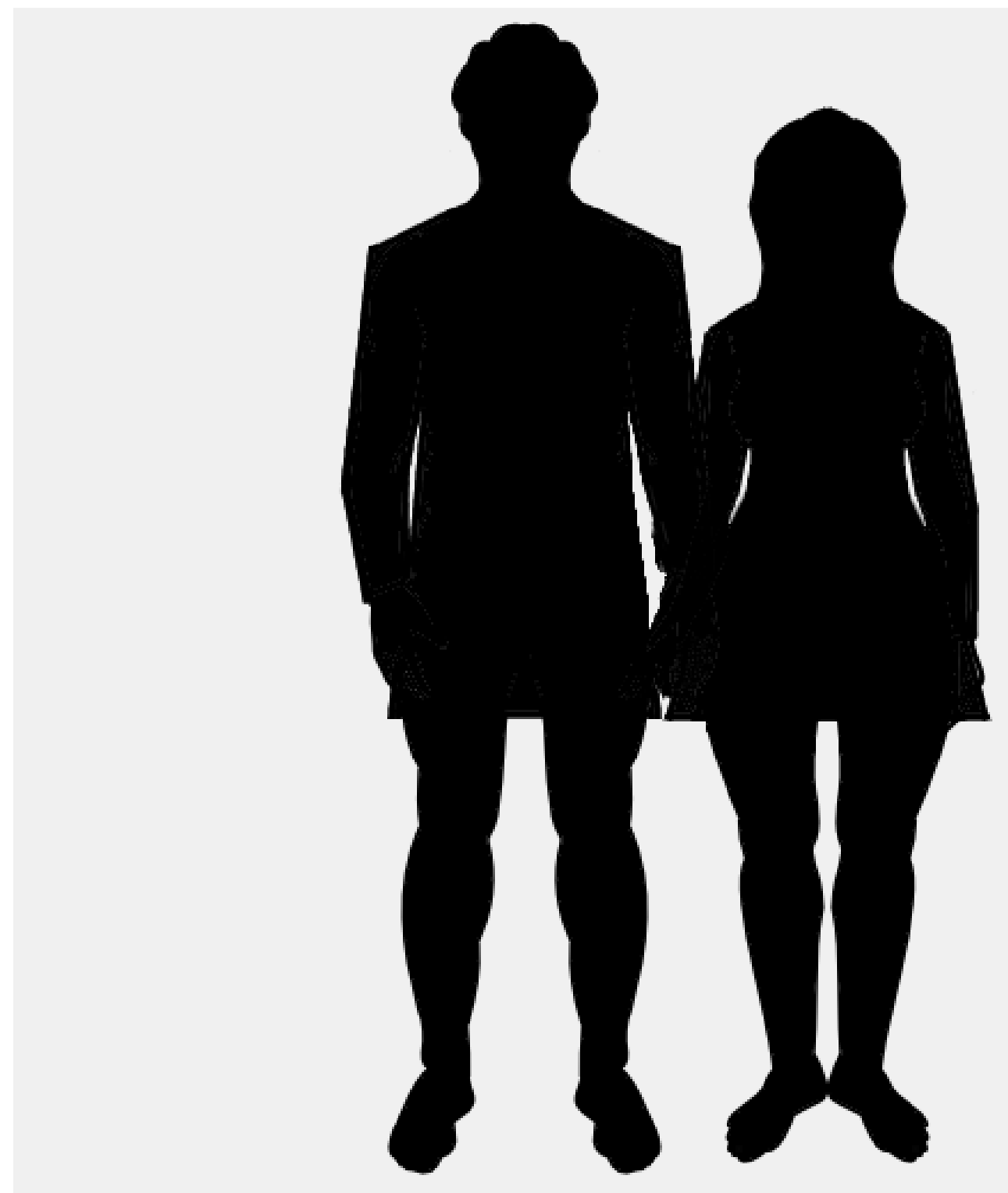

- How aroused are you by the above image? 
- How bad is it to help a friend?

- Your Gender

Ten level Likert scale labels ('How aroused are you by the above image?')

- 1. not aroused at all

- 10. extremely aroused

Multiple Choice Options ('How bad is it to help a friend?')

- somewhat bad

- fairly bad

- quite bad

- very bad

- not bad at all

- extremely bad

Multiple Choice Options (gender)

- male

- female 


\section{Survey S4A (USA participants)}

Survey Title: "Affect Survey"

Instructions:

"Overview - This survey shows a series of images and asks you to rate the level of arousal you experience.

Process - Read and answer the questions to the best of your ability.

Thank you! - Thank you for your honest answers in this task.

(Note this survey is used for scientific research. There are 5 questions in total, and it should take approximately 1-2 minutes to complete carefully)." 


\section{Questions}

Manipulation 1 (tight clothing):

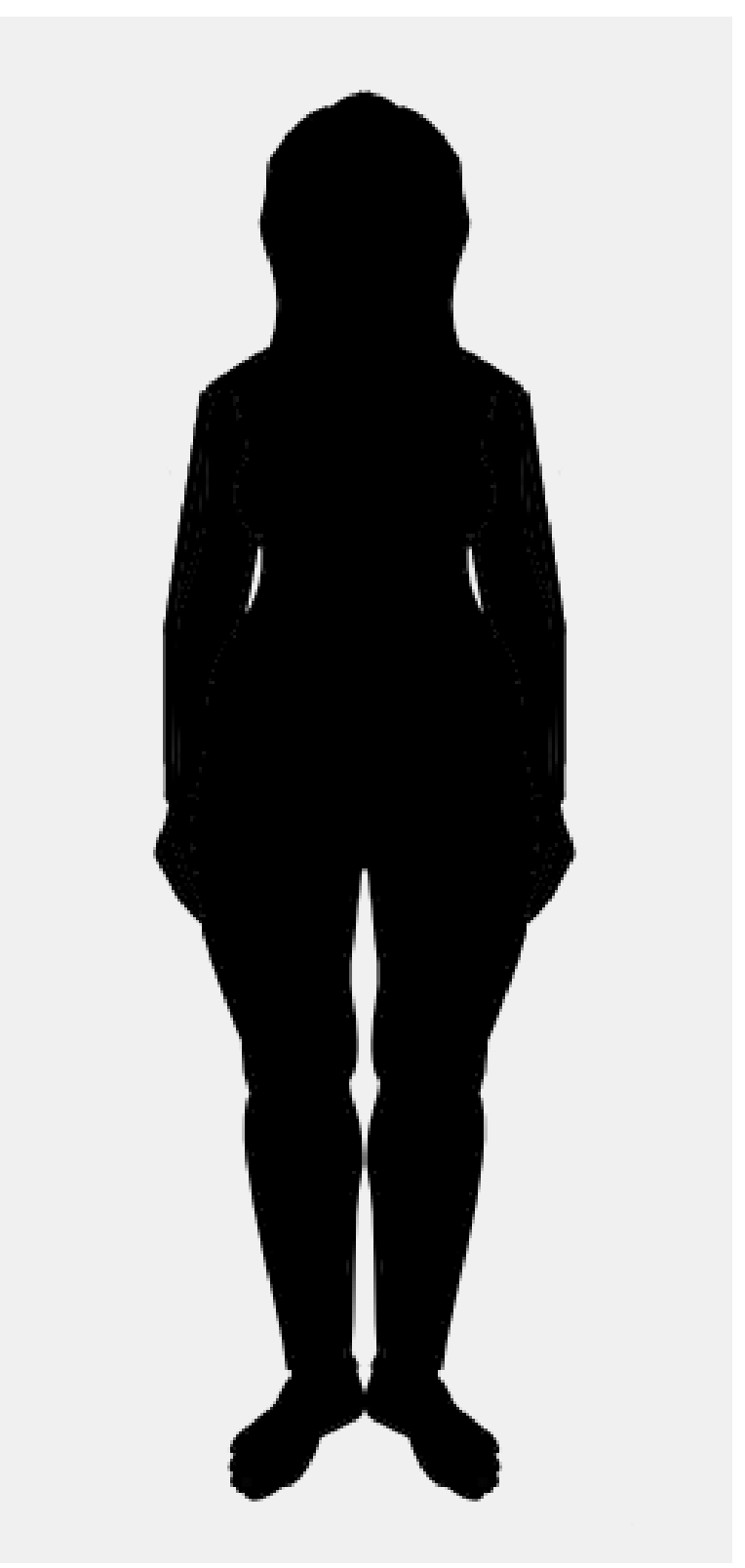

- How aroused are you by the above image? 


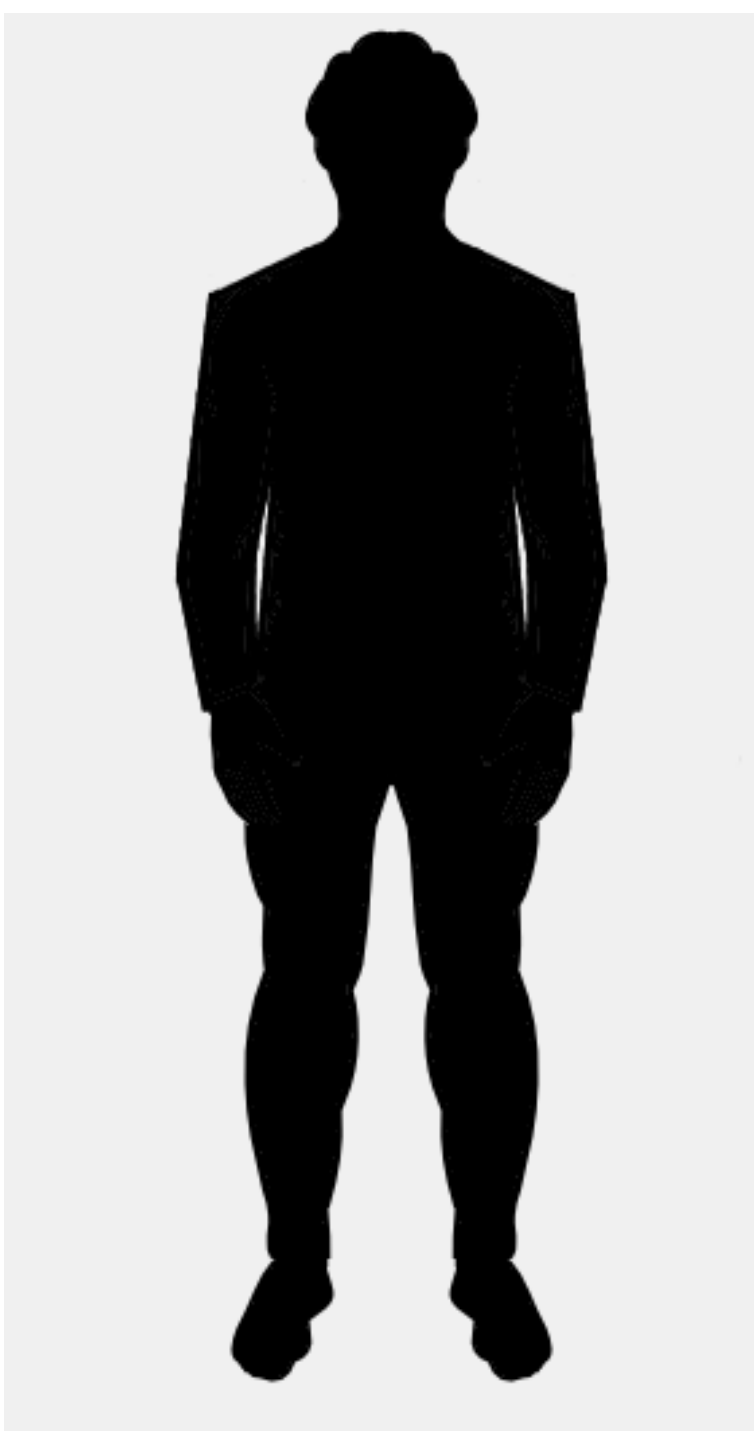

- How aroused are you by the above image? 


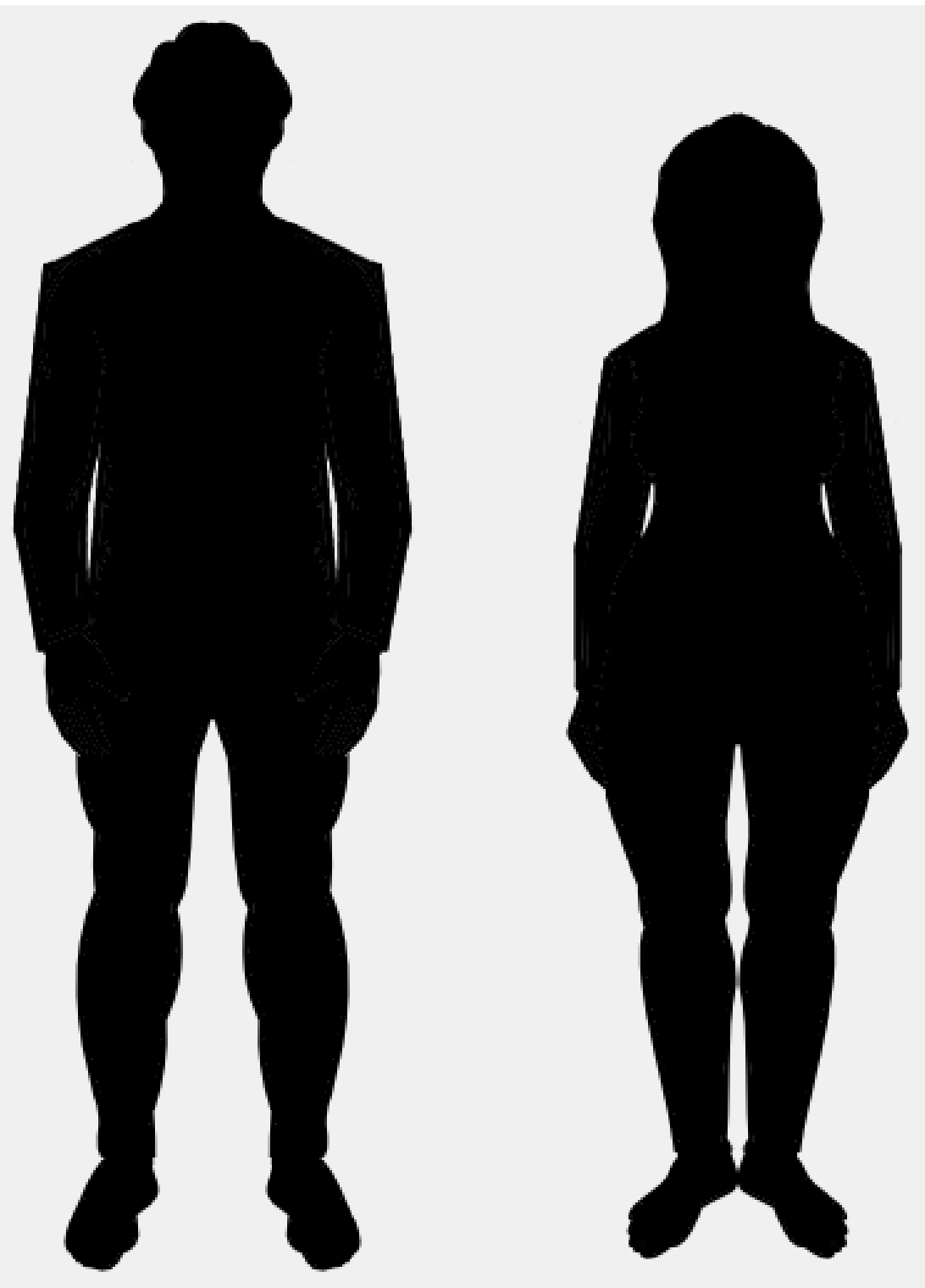

- How aroused are you by the above image? 
Manipulation 2 (short robe):

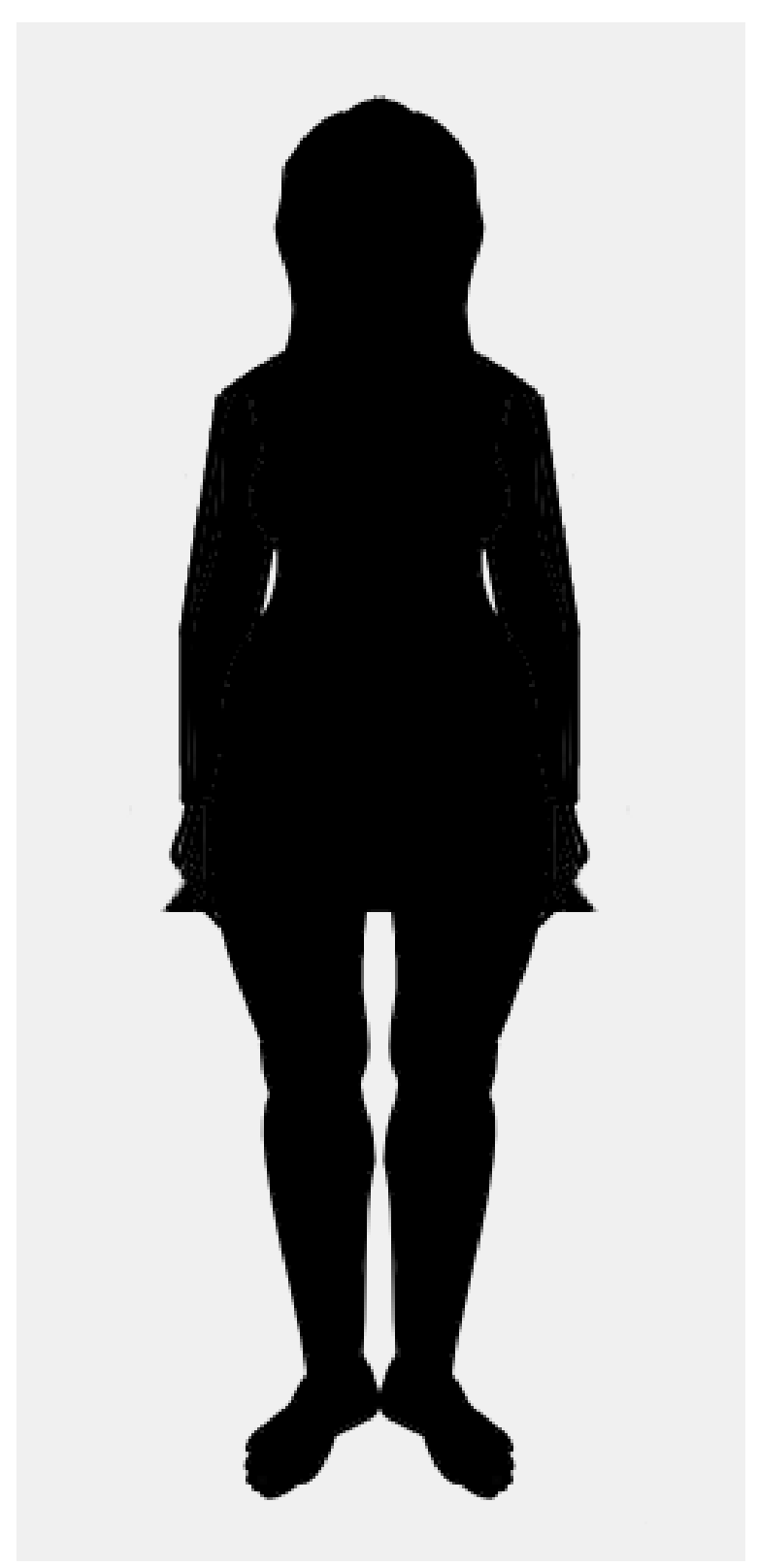

- How aroused are you by the above image? 


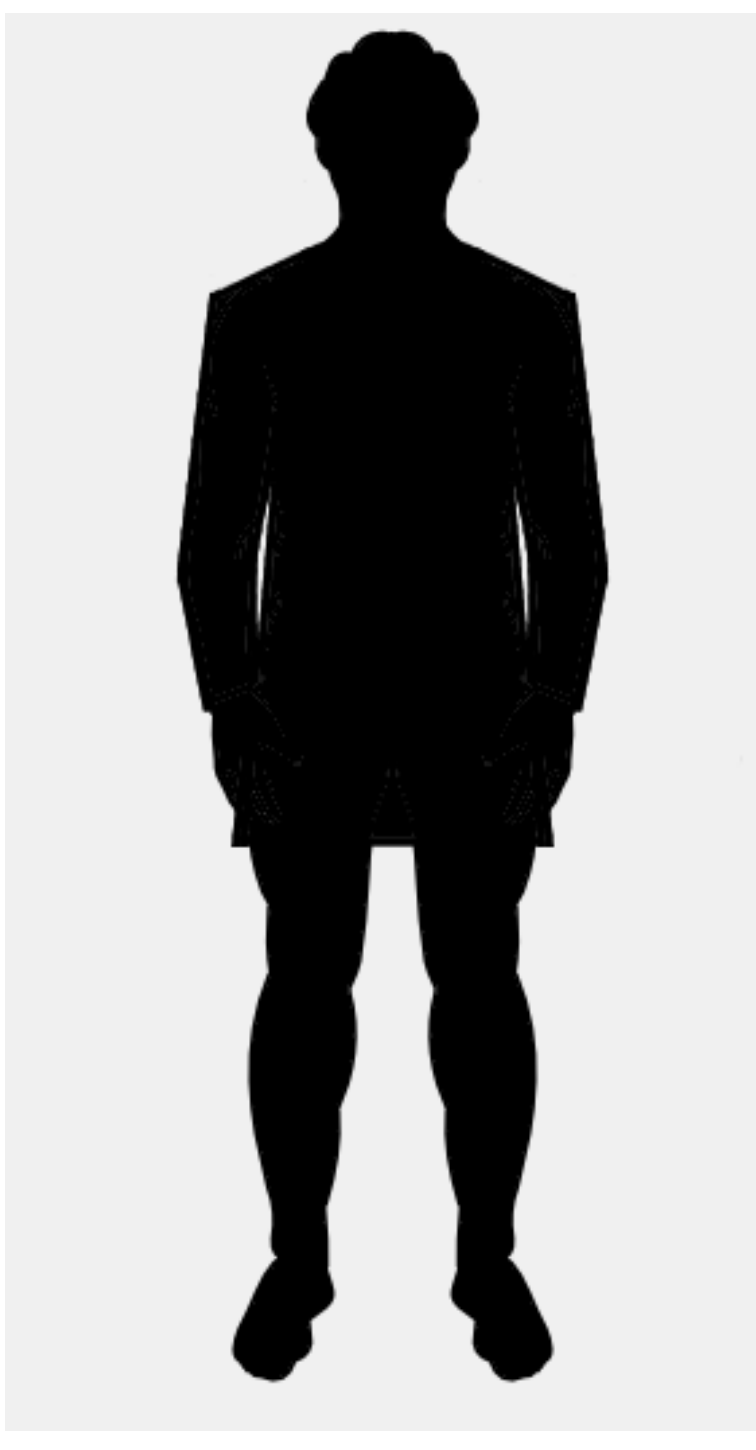

- How aroused are you by the above image? 


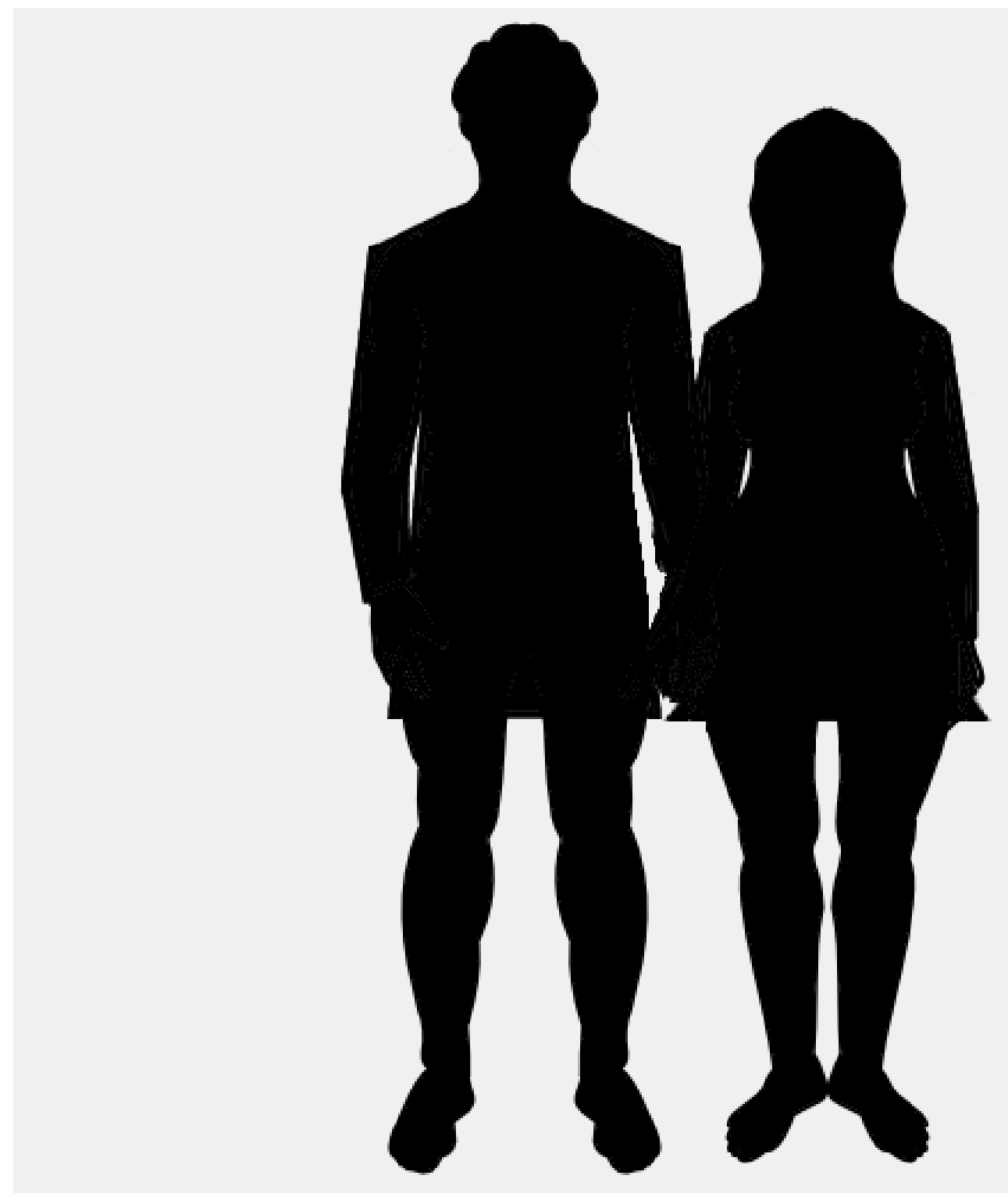

- How aroused are you by the above image? 
- How wrong is it to help your mother?

- Your Gender

Ten level Likert scale labels ('How aroused are you by the above image?')

- 1. not aroused at all

- 10. extremely aroused

Multiple Choice Options ('How wrong is it to help your mother?')

- somewhat wrong

- fairly wrong

- quite wrong

- not wrong at all

- very wrong

- extremely wrong

Multiple Choice Options (gender)

- male

- female 


\section{Survey S4B (USA participants)}

Survey Title: "Affect Survey"

Instructions:

"Overview - This survey shows a series of images and asks you to rate the level of arousal you experience.

Process - Read and answer the questions to the best of your ability.

Thank you! - Thank you for your honest answers in this task.

(Note this survey is used for scientific research. There are 5 questions in total, and it should take approximately 1 minute to complete carefully)." 


\section{Questions}

Manipulation 1 (tight clothing):

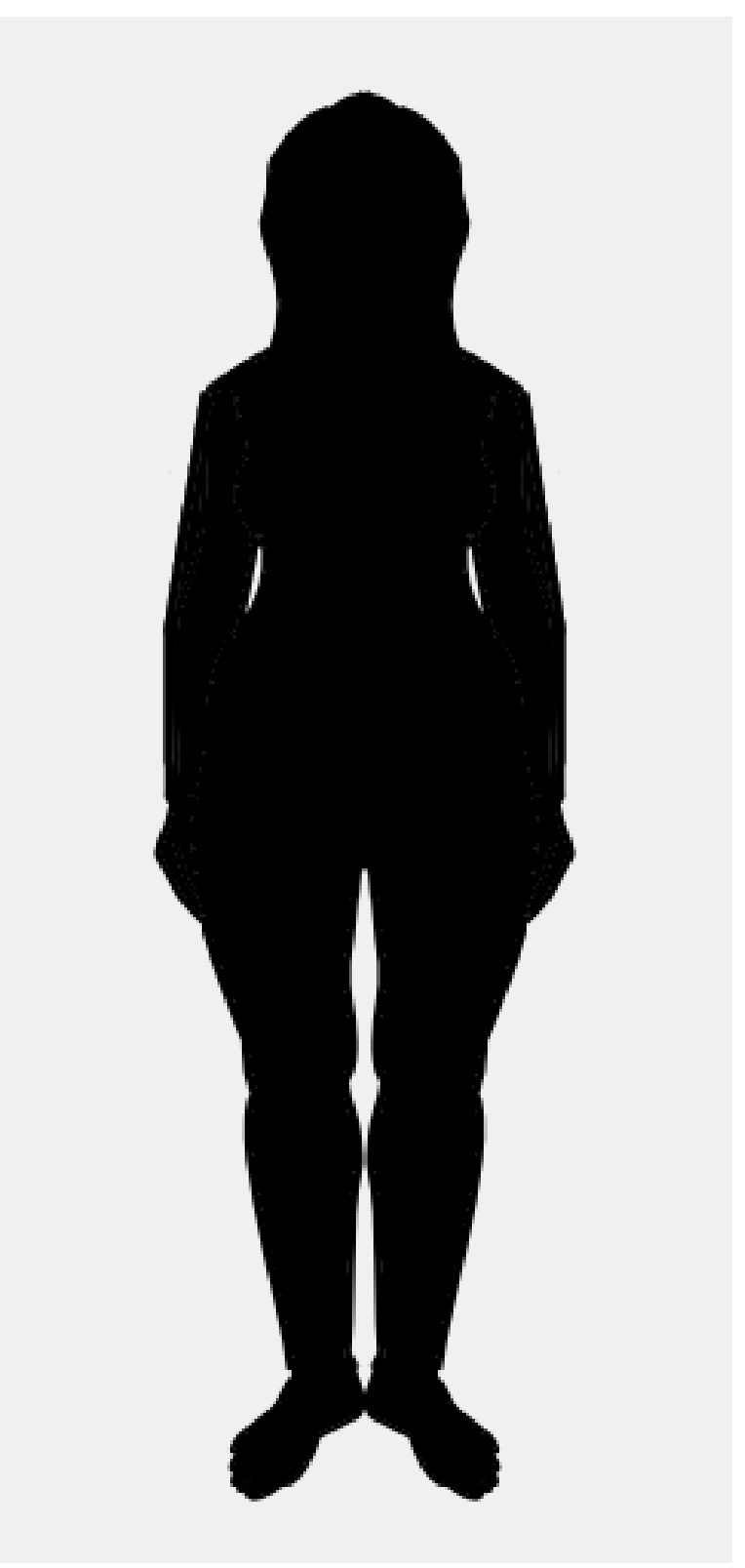

- How aroused are you by the above image? 


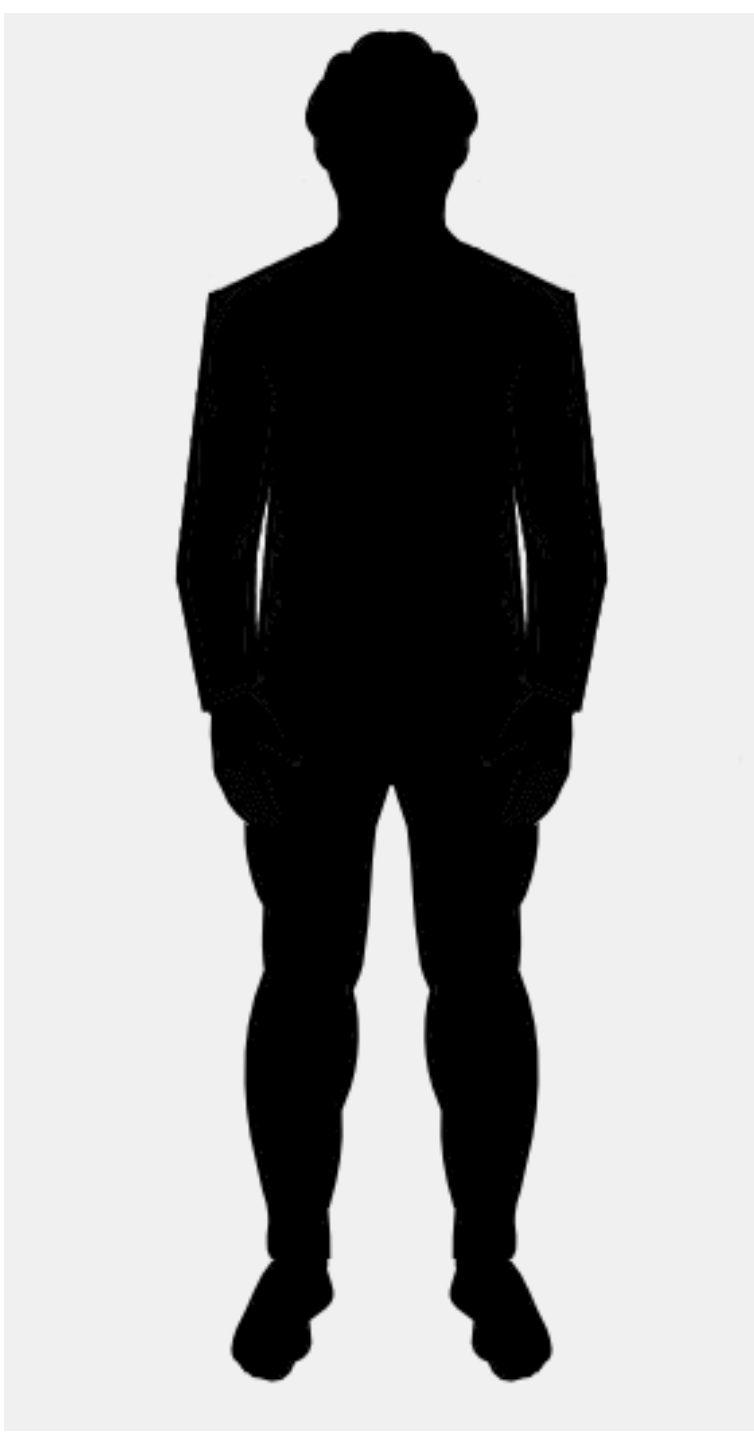

- How aroused are you by the above image? 


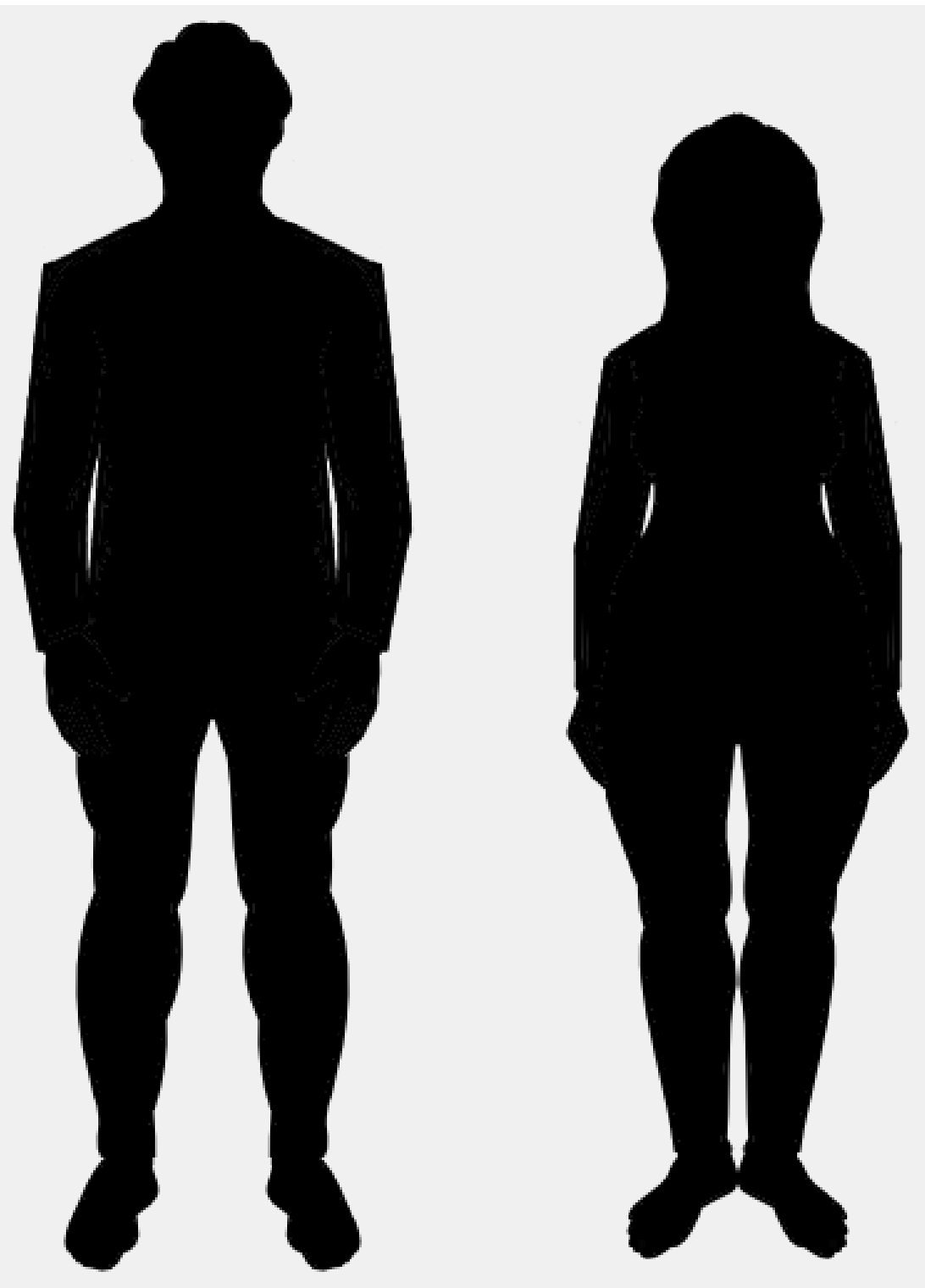

- How aroused are you by the above image? 
Manipulation 2 (short robe):

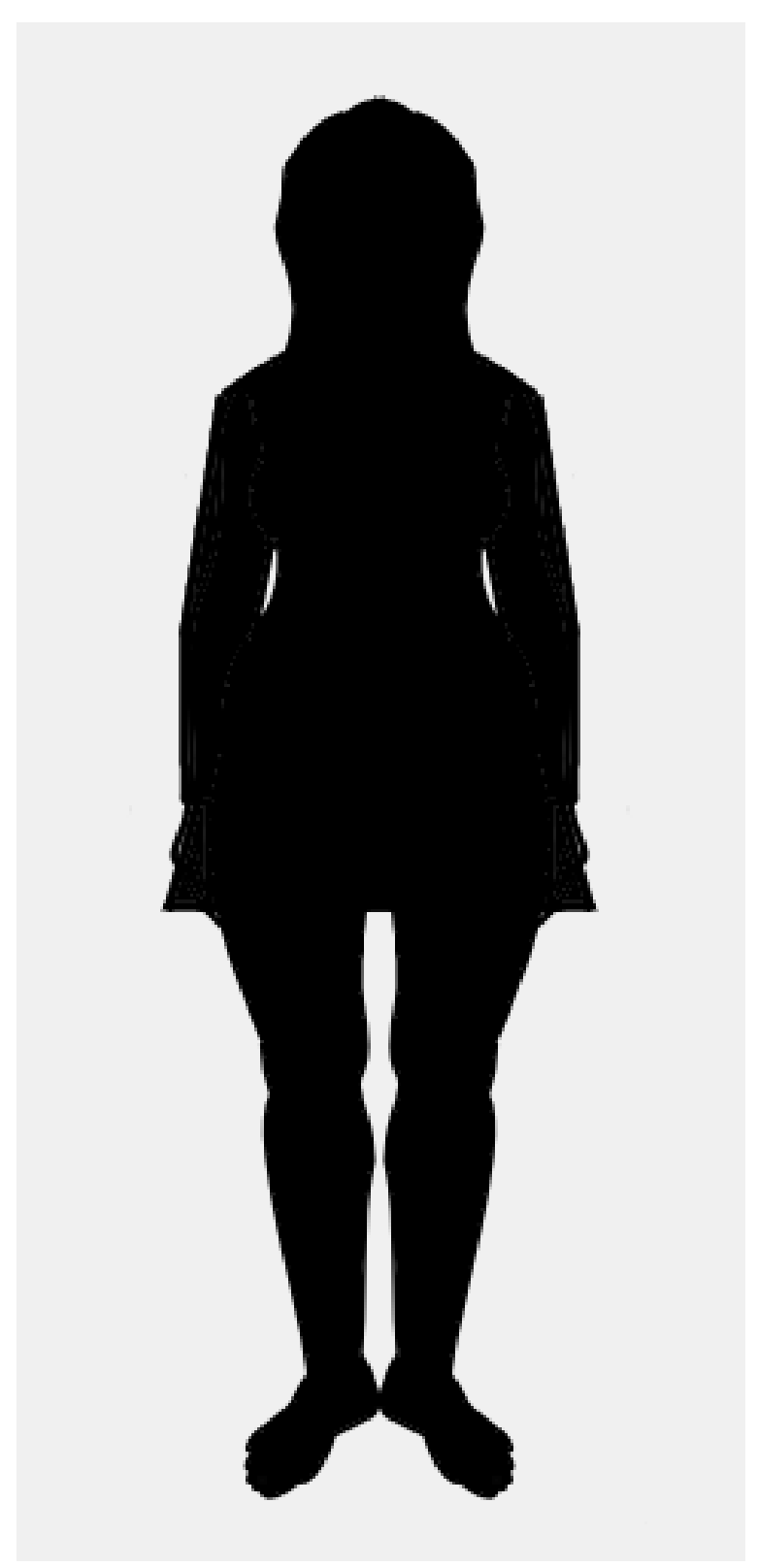

- How aroused are you by the above image? 


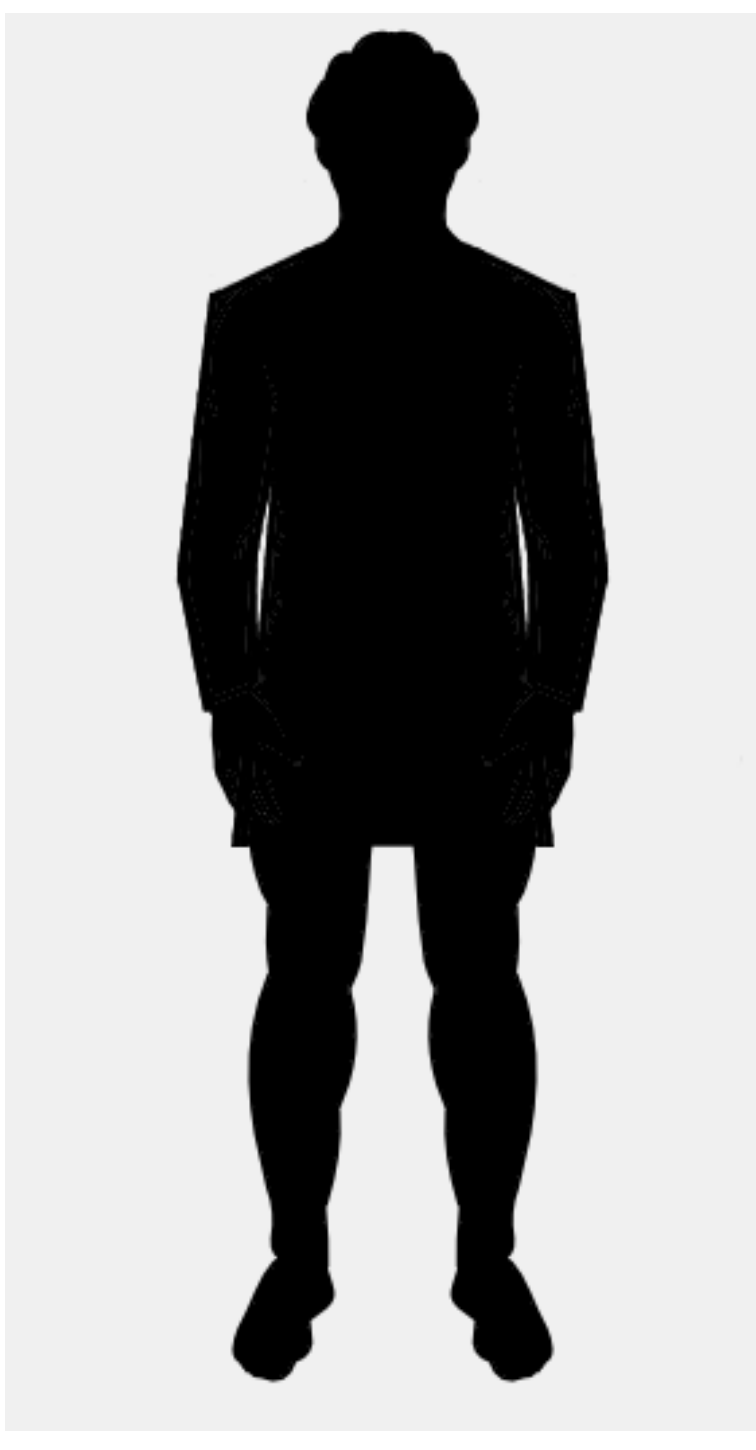

- How aroused are you by the above image? 


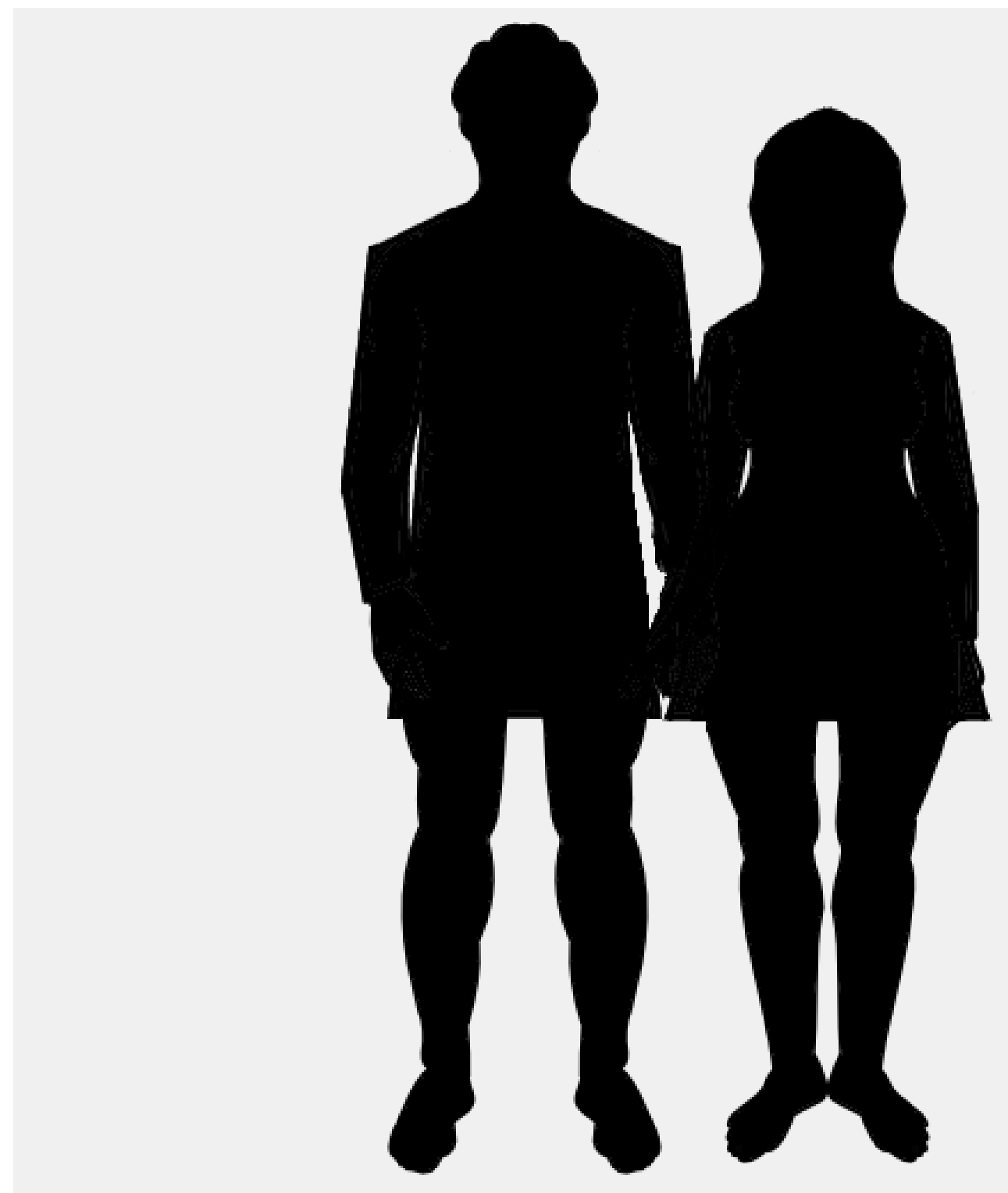

- How aroused are you by the above image? 
- What color is the sky ordinarily?

- Your Gender

Ten level Likert scale labels ('How aroused are you by the above image?')

- 1. not aroused at all

- 10. extremely aroused

Multiple Choice Options ('What color is the sky ordinarily?')

- green

- blue

- orange

- red

- yellow

- purple

Multiple Choice Options (gender)

- male

- female 


\section{S3 Appendix - Bad data filtering}

Given the nature of the crowd source environment, a significant proportion of participants were anticipated to not read and/or answer the questions correctly (to save time while still collecting a survey participation payment). Such false participant data is identified here as 'bad data'. Bad data was filtered by including a dummy question in each survey. Study S1A asked; "How good is it for a person to rob a bank?" Studies S1B, S2 and S3 asked; "How bad is it to help a friend?" Study S4A asked, "How wrong is it to help your mother?" Study S4B asked, "What color is the sky ordinarily?" The number of bad data entries was estimated based on the number of participants

responding to this question with anything but "not bad at all"/"not wrong at all"/"blue" (out of a total of 6 possible response options). The location of this correct option was shifted between surveys.

Studies S1A, S1B, S2, S3 part B, S4A, and S4B were conducted with a moderate CrowdFlower contributor level performance threshold ('Level 2': $80 \%$ accuracy) given the large sample sizes in general required. Study S3 part A was conducted with the highest CrowdFlower contributor level performance threshold ('Level 3': $85 \%$ accuracy). 